\section{Arrkivoc

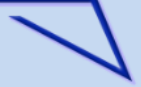

Archive for

Organic Chemistry
The Free Internet Journal

for Organic Chemistry
Paper

Arkivoc 2019, part iv, 0-0

to be inserted by editorial office

\title{
Synthesis of nucleoside analogues using acyclic diastereoselective reactions
}

\author{
Tommy Lussier, ${ }^{\text {a,b }}$ Marie-Ève Waltz, ${ }^{a}$ Garrett Freure, ${ }^{a}$ Philippe Mochirian, ${ }^{a}$ Starr Dostie, ${ }^{a}$ \\ Michel Prévost, ${ }^{\mathrm{a}}$ and Yvan Guindon*a,b \\ ${ }^{a}$ Bio-organic Chemistry Laboratory, Institut de Recherches Cliniques de Montréal (IRCM), Montréal, \\ Québec, H2W 1R7, Canada \\ ${ }^{b}$ Department of Chemistry, Université de Montréal, Montréal, Québec, H3C 3J7, Canada \\ Email: vvan.quindon@ircm.qc.ca
}

This paper is dedicated to Professor Stephen Hanessian, a mentor, colleague and friend

Received 12-03-2018

Accepted 03-11-2019

Published on line 04-29-2019

\section{Abstract}

The design of novel xylo-like nucleoside analogues bearing a $\mathrm{C3}^{\prime}$ all-carbon quaternary center and a $\mathrm{C2}^{\prime}$ hydroxy substituent is described. Synthesis of this scaffold makes use of highly diastereoselective transformations on acyclic substrates. Central to the approach is formation of a 2,4-syn cyanohydrin from cyanide addition onto an aldehyde through a proposed seven-membered ring chelate using a bidentate Lewis acid. In addition, a highly diastereoselective Mukaiyama aldol reaction, an intramolecular radical atom cyclization, and thioaminal formation are used to generate this novel molecule. A series of related nucleoside analogues are being tested as antiviral and anticancer agents.

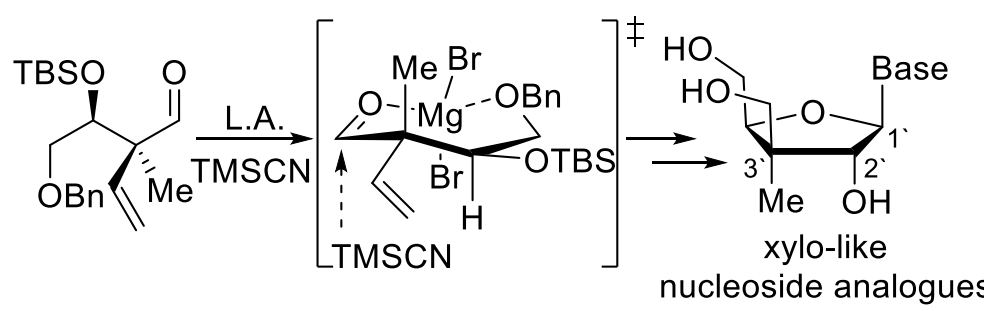

Keywords: Nucleoside analogues, xylo-like scaffolds, diastereoselective, acyclic, cyanohydrin, all-carbon quaternary stereo-center 


\section{Introduction}

The implication of endogenous nucleosides and nucleotides in numerous biological pathways has, not surprisingly, inspired the development of various analogues as inhibitors of tumor growth and viral replication. ${ }^{1,2,3}$ Exploring the biological profiles of novel nucleoside analogues remains crucial, as shown by the recent approval of highly efficient antiviral drugs. ${ }^{4}$ We have initiated the syntheses of novel nucleoside analogues possessing an all-carbon quaternary stereogenic center at C3'. $^{5-7}$ It is proposed that this chiral center could enhance target specificity in addition to providing opportunities for the incorporation of different pharmacophores. As illustrated in Figure 1, the hydroxymethyl substituent at C3' can have either a xylo/lyxo( $\beta$-face) or ribo/arabino- ( $\alpha$-face) like orientation. The syntheses of $C 2$ 'fluoro analogues in which the hydroxymethyl substituent is located on the $\alpha$-face (ribo-like scaffolds) has been previously reported by our group. ${ }^{8}$ Herein, we describe the synthesis of xylo-like analogues with a C2'-hydroxy group. Incorporation of a C3'-hydroxymethyl substituent with a $\beta$-orientation is a feature of apio-nucleosides, a class of analogues in which the hydroxymethyl normally found at C4' is shifted to C3'..$^{9-11}$ Presently, our scaffolds are being tested for their antiviral and anticancer properties; however, this novel xylo-like scaffold may also show potential in the design of new fungicides ${ }^{12}$ and insecticides, ${ }^{13}$ as antimicrobial agents ${ }^{14}$ and acetylcholinesterase inhibitors ${ }^{15}$ all of which contain a xylo-furanoside core.

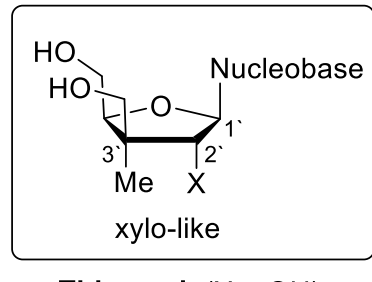

This work $(\mathrm{X}=\mathrm{OH})$

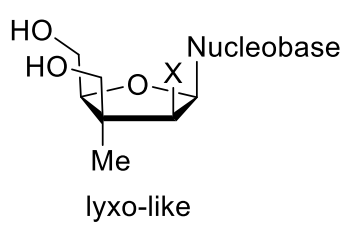

lyxo-like

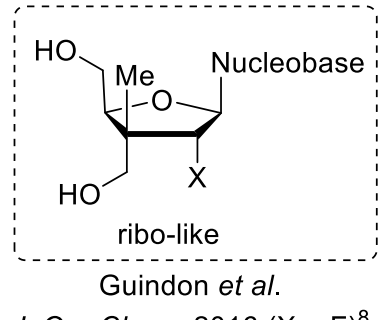

J. Org Chem. $2016(X=F)^{8}$

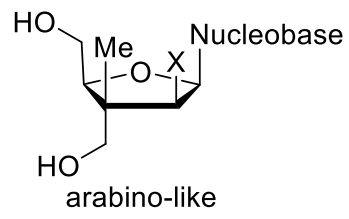

arabino-like

Figure 1. Nucleoside analogues bearing an all-carbon stereogenic center at C3'.

To construct our novel scaffold (1), a series of diastereoselective acyclic transformations were developed (Scheme 1). Using our two-step acyclic approach for the synthesis of nucleoside analogues, 1',2'-trans furanoside 2, was formed from a kinetically controlled intramolecular cyclization of chiral thioaminal 3. This 1,2-syn thioaminal resulted from diastereoselective nucleobase addition onto dithioacetal 4.
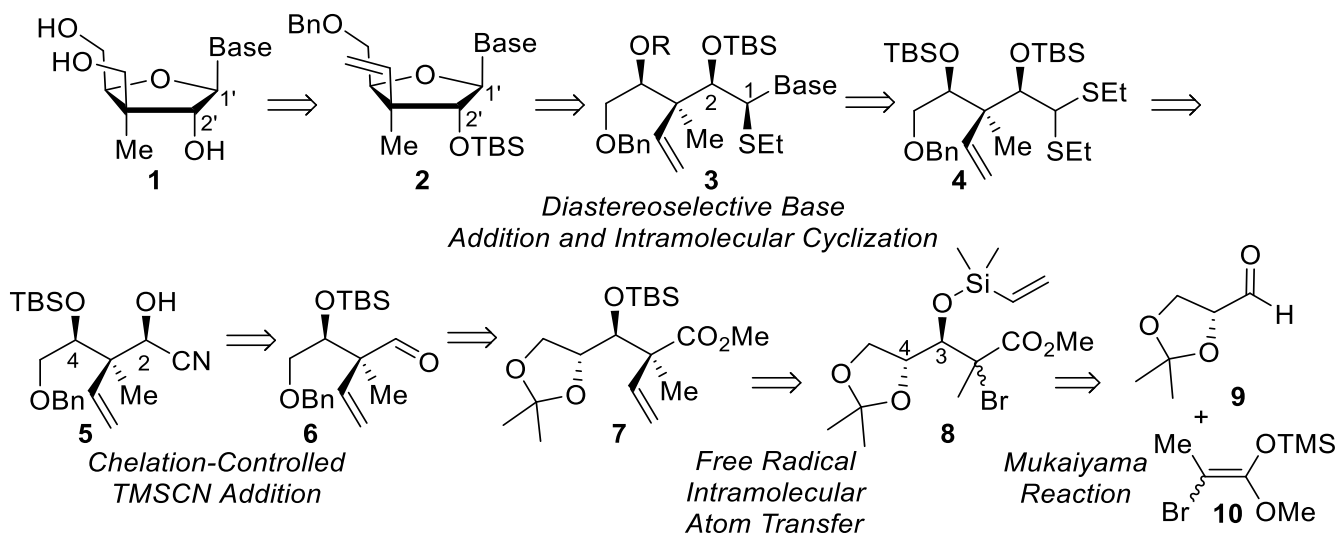

Scheme 1. Retrosynthetic analysis of xylo-like analogues. 
Central to our synthetic approach was a Lewis acid controlled diastereoselective cyanide (TMSCN) addition onto aldehyde 6 through a proposed seven-membered ring chelate to generate the C2'-hydroxy substituent from formation of the acyclic cyanohydrin 5. An intramolecular silicon tethered free radical-based vinylation was used to construct the all-carbon stereogenic center in 7, the substrate of which was accessed from a Mukaiyama aldol reaction between aldehyde $\mathbf{9}$ and enoxysilane $\mathbf{1 0}$.

\section{Results and Discussion}

The reaction sequence to generate the targeted family of nucleoside analogues began with construction of the all-carbon quaternary center (Scheme 2). The key precursor $\mathbf{8 a , b}$ for the intramolecular radical atom transfer cyclization was efficiently accessed by a three-step sequence involving a Mukaiyama aldol reaction ${ }^{16,17}$ between 2,3-isopropylidene-D-glyceraldehyde 9 and enoxysilanes 10 in the presence of $\mathrm{MgBr}_{2} \cdot \mathrm{OEt}_{2}{ }^{7}$ Treatment of the crude reaction mixture with HF-pyridine was necessary to remove undesired TMS protection of the oxygen at C3. The vinyldimethylchlorosilane moiety was then installed on the resulting free alcohols to provide the 3,4-anti $(\mathbf{8 a}, \mathbf{b})$ and 3,4-syn $(\mathbf{1 1 a}, \mathbf{b})$ bromides in a 10:1 ratio and excellent yield $(52 \%)$ over the three steps. Preference for the 3,4-anti stereochemistry has been proposed to occur through a Felkin-Ahn transition state. ${ }^{7,18-21}$ The steric hindrance imposed by the isopropylidene moiety of aldehyde $\mathbf{9}$ is likely to prohibit chelate formation between the aldehyde and $\alpha$-oxygen.

\section{Mukaiyama Aldol Reaction:}
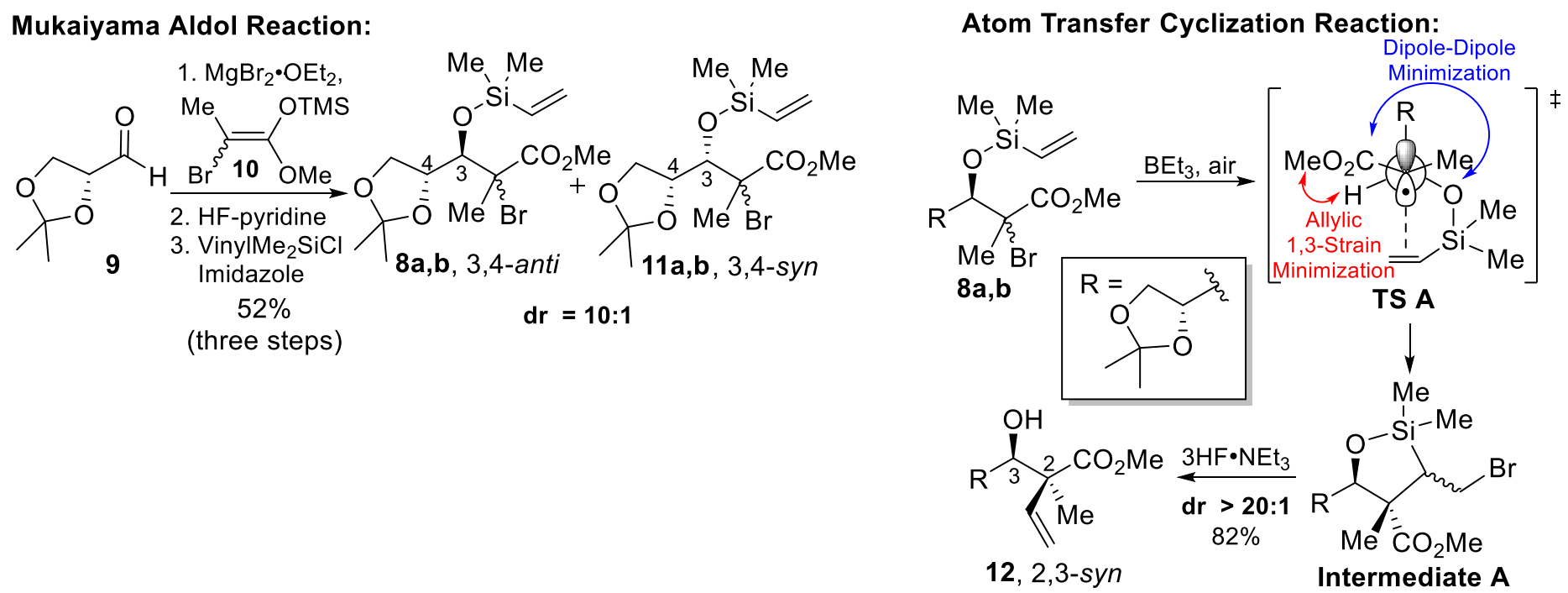

Scheme 2. Synthesis of the all-carbon quaternary center in $\mathbf{1 2 .}$

The free radical tandem cyclization/elimination reaction developed by our laboratory ${ }^{22}$ was then performed on $\mathbf{8} \mathbf{a}, \mathbf{b}$ in the presence of $\mathrm{BEt}_{3} / \mathrm{O}_{2}$ (Scheme 2). The reaction was shown to proceed through a fivemembered ring intermediate $\mathbf{A}$ (5-exo-trig) bearing a mixture of primary bromides. ${ }^{22}$ The stereochemistry of the newly formed quaternary center was proposed to originate from transition state $A$ that minimizes intramolecular dipole-dipole interactions and allylic 1,3-strain. ${ }^{23}$ The five-membered silyloxy ether intermediates (A) were cleaved upon treatment with $3 \mathrm{HF} \cdot \mathrm{NEt}_{3}$ to give the 2,3-syn product 12 as the only observable diastereomer (>20:1) in $82 \%$ yield (see supporting information for stereochemical proofs). 
Derivatization of 12 towards aldehyde $\mathbf{6}$ was achieved by first carrying out silylation of the secondary alcohol (Scheme 3). Reduction of the methyl ester and subsequent benzoylation generated the protected primary alcohol 13 in 68\% yield over three steps. Cleavage of the acetonide and oxidation of the 1,2-diol using periodic acid led to an aldehyde that was immediately reduced to the corresponding primary alcohol 14 (85\% yield over two steps), which was then protected with a benzyl group to give 15 . Deprotection of the primary benzoate with DIBAL-H followed by DMP oxidation provided aldehyde 6 .
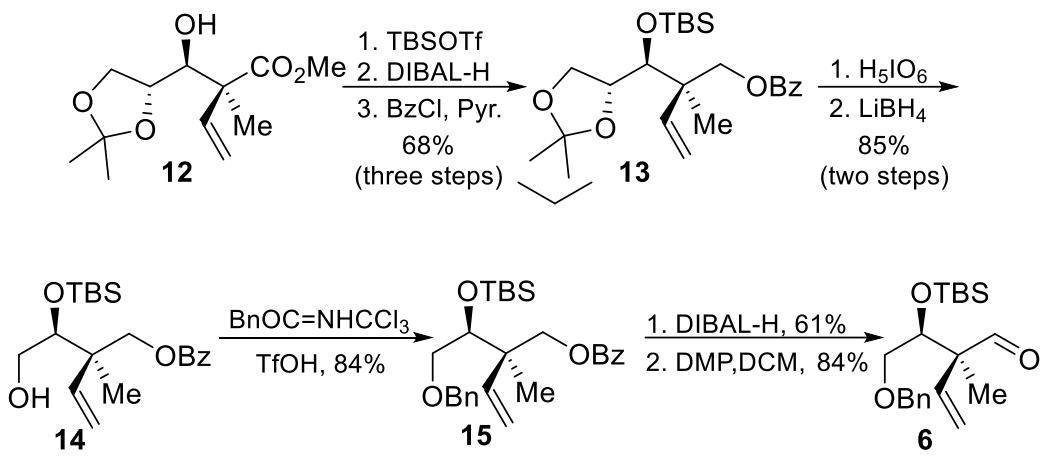

Scheme 3. Synthesis of aldehyde 6 bearing an $\alpha$-quaternary center and a $\beta$-hydroxy group.

The diastereoselective addition of cyanide ${ }^{24,25}$ onto aldehyde 6 was next studied. The desired xylo-like scaffold of the targeted nucleoside analogue (1) required a syn stereochemistry between the substituents at C2 and C4 of the resulting cyanohydrin (5). 1,2-Induction using a monodentate Lewis acid (TS B and TS C, Scheme 4) was expected to be poor, since two of the substituents of the quaternary center (methyl and vinyl) are sterically similar. The possibility of using the stereogenic secondary $\beta$-hydroxy group of the acyclic aldehyde was therefore considered.

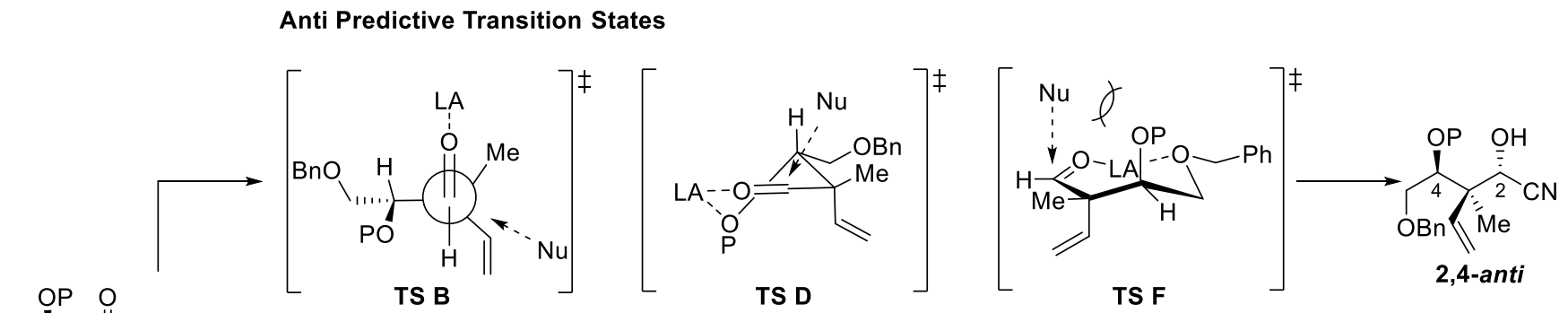<smiles>C=CC(C)(C=O)[C@H](O)COCC#N</smiles>

Syn Predictive Transition States

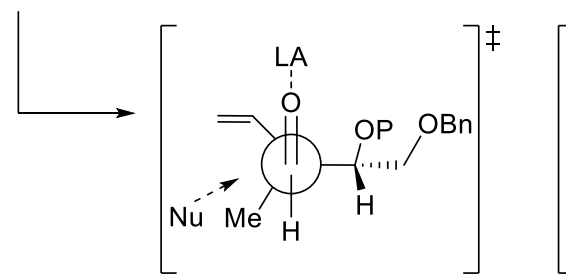

TS C

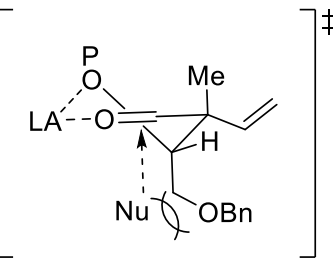

TS E

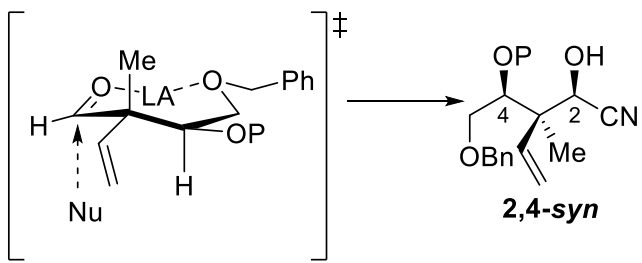

TS G

$\mathrm{P}=$ Protecting Group; $\mathrm{LA}=$ Lewis Acid; $\mathrm{Nu}=$ Nucleophile $(\mathrm{TMSCN})$

Scheme 4. Transition states for diastereoselective cyanohydrin formation. 
A bidentate Lewis acid could generate different reactive chelate intermediates. Chelation between the $\beta$-hydroxy group and the aldehyde is expected to give preference for the undesired 2,4-anti relationship through TS $\mathbf{D}$, which avoids steric clash between the incoming nucleophile and the $-\mathrm{CH}_{2} \mathrm{OBn}$ found in TS E (2,4syn predictive transition state). However, the presence of the $\alpha$-quaternary center could decrease the energy difference between TS $\mathbf{D}$ and TS E, the former having two gauche interactions between the substituents of the $\alpha$ - and $\beta$-stereogenic centers. Formation of a seven-membered ring intermediate (TS F and TS G) between the primary oxygen and the aldehyde could allow more flexibility for chelate formation and alleviate steric interactions between the incoming nucleophile and the quaternary center. Formation of the desired 2,4-syn cyanohydrin (TS G) in which nucleophilic attack occurs on the bottom face should be favored to avoid eclipsing interactions between the incoming nucleophile and the axial methyl of the quaternary center in TS $\mathbf{G}$ or with the $\beta$-hydroxy group in TS F.

To prevent potential competition between six- and seven-membered ring chelates, a bulky TBS group was incorporated onto the $\beta$-hydroxy of aldehyde 6 . Precomplexation of aldehyde 6 with an excess of $\mathrm{MgBr}_{2} \cdot \mathrm{OEt}_{2}{ }^{24}$ was followed by addition of the cyanide source (TMSCN) at $-15{ }^{\circ} \mathrm{C}$ (Scheme 5). Cyanohydrin 5 was formed in an 8:1 ratio in favor of the 2,4 syn isomer in excellent yield (83\% over two steps). It is noteworthy that the reaction of TMSCN and the aldehyde alone in DCM resulted in recuperation of the starting material, thus highlighting the need for a source of Lewis acid. Cyanide addition did occur in the presence of a Lewis base $\left(\mathrm{NEt}_{3}\right.$ or $\left.\mathrm{NH}(i-\mathrm{Pr})_{2}\right)$ to furnish OTMS protected cyanohydrins (results not shown), albeit with low diastereoselectivity. ${ }^{26}$ These observations are in accordance with precedent examples demonstrating that a Lewis acidic or basic species is needed to activate the TMSCN through formation of a pentacoordinate siliconate ion. ${ }^{27,28}$

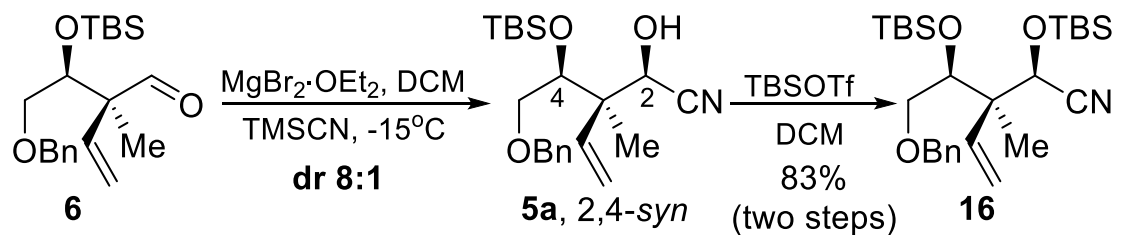

Scheme 5. Synthesis of 2,4-syn cyanohydrin.

To validate our initial hypotheses for 2,4-syn selectivity, a model study was undertaken at $0{ }^{\circ} \mathrm{C}$ (Table 1 ). As can be seen in entry 1, a 6:1 ratio in favor of the syn cyanohydrin was obtained at this temperature. When the reaction was performed with a monodentate Lewis acid, $\mathrm{BF}_{3} \cdot \mathrm{OEt}_{2}$, an expected low diastereoselectivity of 1.5:1 was observed in favor of the 2,4-syn product (entry 2). Interestingly, when the TBS $\beta$-hydroxy protecting group was replaced by a benzyl or $p$-methoxybenzyl group, (aldehydes 17 and 19), preference for the 2,4-syn cyanohydrin was maintained (entries 3 and 4) with $\mathrm{MgBr}_{2} \cdot \mathrm{OEt}_{2}$, even though competing TS $\mathbf{D}$ could be at play. With aldehyde 21, bearing a free $\beta$-hydroxy group (entry 5), a reversal of selectivity in favor of the 2,4-anti product was indeed noted suggesting reaction through TS $\mathbf{D}$.

Suppressing the possibility of creating a seven-membered ring chelate by replacing the primary benzyl protecting group with a bulky silyl ether, as in aldehyde 23, abolished the diastereoselection noted before $(1.5: 1$, entry 6$)$. Similarly, when the benzyl ether was replaced by its carbon equivalent as in $\mathbf{2 5}$, a decrease in selectivity was observed (2.5:1, entry 7). To ensure that this loss of selectivity was not the result of having an a-gem-dimethyl instead of the stereogenic center, we prepared 27 and observed a high syn preference (11:1 entry 8 versus $6: 1$ entry 1 ) which suggests a greater interaction between the incoming nucleophile and olefin 
in TS G. Taken together, these results support the intermediacy of a 7-membered ring chelate (TS G) $)^{29-31}$ in inducing 2,4-syn diastereoselectivity for cyanohydrin formation in the presence of $\mathrm{MgBr}_{2} \cdot \mathrm{OEt}_{2}$.

Interestingly, with the use of a titanium Lewis acid (Table 1, entries 9-13), formation of the 2,4-anti cyanohydrin could be favored. When aldehyde 19 bearing a $\beta$-PMB was reacted with 1.1 equivalents of $\mathrm{TiCl}_{3}(\mathrm{O} i \mathrm{Pr})$, a $1: 1$ ratio of syn and anti cyanohydrins were formed (entry 9 ) in which the $\beta$-PMB was cleaved. Upon increasing the equivalents of $\mathrm{TiCl}_{3}(\mathrm{OiPr})$ to 2.5 (entries 10 and 11) a 1:6 ratio was obtained in favor of the 2,4-anti diol 22b. Not surprisingly, with a $\beta$-OTBS, preference for the 2,4-syn cyanohydrin 5a was maintained (3:1 ratio, entry 12), favoring cyanation through 7-membered chelate TS G or monodentate activation.

Table 1. Diastereoselective cyanohydrin formation.

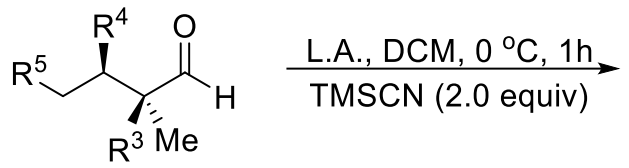

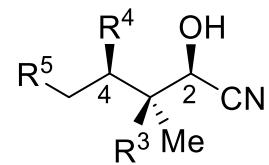

a, 2,4-syn

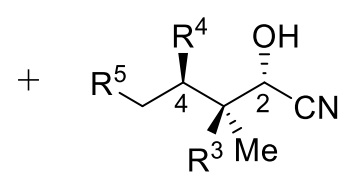

b, 2,4-anti

\begin{tabular}{|c|c|c|c|c|c|c|c|c|c|}
\hline \multicolumn{2}{|c|}{ Entry Aldehyde } & \multirow{2}{*}{$\frac{\text { L.A. }}{\mathrm{MgBr}_{2} \cdot \mathrm{OEt}_{2}}$} & \multirow{2}{*}{$\frac{\text { Equiv. L.A. }}{5.0}$} & \multirow{2}{*}{$\frac{R^{5}}{O B n}$} & \multirow{2}{*}{$\frac{\mathrm{R}^{4}}{\text { OTBS }}$} & \multirow{2}{*}{$\frac{\mathrm{R}^{3}}{\text { vinyl }}$} & \multirow{2}{*}{$\frac{\text { Ratio }(\mathbf{a}: \mathbf{b})^{\mathrm{b}}}{6: 1}$} & \multirow{2}{*}{$\begin{array}{c}\text { Cyanohydrin } \\
5 \mathbf{a}, \mathbf{b}\end{array}$} & \multirow{2}{*}{$\begin{array}{c}\text { Yield (\%) } \\
79\end{array}$} \\
\hline 1 & 6 & & & & & & & & \\
\hline 2 & 6 & $\mathrm{BF}_{3} \cdot \mathrm{OEt}_{2}$ & 1.5 & $\mathrm{OBn}$ & OTBS & vinyl & $1.5: 1$ & $5 a, b$ & 63 \\
\hline 3 & 17 & $\mathrm{MgBr}_{2} \cdot \mathrm{OEt}_{2}$ & 5.0 & OBn & $\mathrm{OBn}$ & vinyl & $8: 1^{c}$ & $18 a, b$ & 81 \\
\hline 4 & 19 & $\mathrm{MgBr}_{2} \cdot \mathrm{OEt}_{2}$ & 5.0 & $\mathrm{OBn}$ & OPMB & vinyl & $8: 1$ & $20 a, b$ & 93 \\
\hline 5 & 21 & $\mathrm{MgBr}_{2} \cdot \mathrm{OEt}_{2}$ & 5.0 & $\mathrm{OBn}$ & $\mathrm{OH}$ & vinyl & $1: 4$ & $22 a, b$ & 89 \\
\hline 6 & 23 & $\mathrm{MgBr}_{2} \cdot \mathrm{OEt}_{2}$ & 5.0 & OTBDPS & OTBS & vinyl & $1.5: 1$ & $24 a, b$ & 93 \\
\hline 7 & 25 & $\mathrm{MgBr}_{2} \cdot \mathrm{OEt}_{2}$ & 5.0 & $\mathrm{CH}_{2} \mathrm{CH}_{2} \mathrm{Ph}$ & OTBS & methyl & $2.5: 1$ & $26 a, b$ & 82 \\
\hline 8 & 27 & $\mathrm{MgBr}_{2} \cdot \mathrm{OEt}_{2}$ & 5.0 & OBn & OTBS & methyl & $11: 1$ & $28 a, b$ & 79 \\
\hline 9 & 19 & $\mathrm{TiCl}_{3}(\mathrm{OiPr})$ & 1.1 & OBn & OPMB & vinyl & $1: 1$ & $22 a, b^{d}$ & ND \\
\hline 10 & 19 & $\mathrm{TiCl}_{3}(\mathrm{OiPr})$ & 2.5 & OBn & OPMB & vinyl & $1: 6$ & $22 a, b^{d}$ & 59 \\
\hline 11 & 21 & $\mathrm{TiCl}_{3}(\mathrm{OiPr})$ & 2.5 & OBn & $\mathrm{OH}$ & vinyl & $1: 6$ & $22 a, b$ & 49 \\
\hline 12 & 6 & $\mathrm{TiCl}_{3}(\mathrm{OiPr})$ & 2.5 & OBn & OTBS & vinyl & $3: 1$ & $5 a, b$ & 68 \\
\hline 13 & 17 & $\mathrm{TiCl}_{3}(\mathrm{OiPr})$ & 2.5 & OBn & OBn & vinyl & $1: 1^{c}$ & $18 a, b$ & 91 \\
\hline
\end{tabular}

a Synthesis of racemic aldehydes 6, 17, 19, 21, 23, 25 and 27 is described in the experimental section along with proofs of structure in the supporting information. The two cyanohydrin diastereomers were separated, deprotected to the corresponding diols and then protected as an acetonide. The relative stereochemistry of the syn and anti-acetonides was determined from 1D NOESY and the ${ }^{13} \mathrm{C}$ chemical shifts of the acetal carbon and the gem-dimethyl substituents. ${ }^{b}$ 2,4-Syn:2,4-anti ratios determined by ${ }^{1} \mathrm{H}$ NMR analysis of the crude reaction mixture. ${ }^{c}$ Relative stereochemistry not determined in this case. ${ }^{d}$ PMB-cleaved, resulting in diol product.

NMR Studies. To help elucidate the preference for 2,4-anti cyanohydrin formation with aldehydes 19 ( $\beta$ $\mathrm{OPMB}$ ) and $21(\beta-\mathrm{OH})$ in the presence of 2.5 equivalents of $\mathrm{TiCl}_{3}(\mathrm{OiPr})$ (entries 10 and 11$)$, low temperature ${ }^{13} \mathrm{C}$ 
NMR spectra of the complexed aldehydes were acquired in $\mathrm{CD}_{2} \mathrm{Cl}_{2}$ (Figure 2). The first observation made was that both aldehydes resulted in similar ${ }^{13} \mathrm{C}$ spectra upon addition of 1.1 equivalents of $\mathrm{TiCl}{ }_{3}(\mathrm{OiPr})$, which indicates that the $\beta$-PMB of aldehyde 19 is cleaved before addition of the nucleophile. ${ }^{32}$ Complex spectra (not shown) were obtained with three new peaks in the carbonyl region. It is likely that these correspond to different intermediates that react unselectively to give mixtures of diastereomers (Table 1, entry 9). However, in the presence of 2.5 equivalents of $\mathrm{TiCl}_{3}(\mathrm{OiPr}$ ) (Figure 2, upper spectrum) there is clearly only one complexed carbonyl species with a significant downfield (11.5 ppm) signal. In addition, the carbons corresponding to C3, C4 and C5 are also all shifted downfield, which supports their involvement in complex formation.
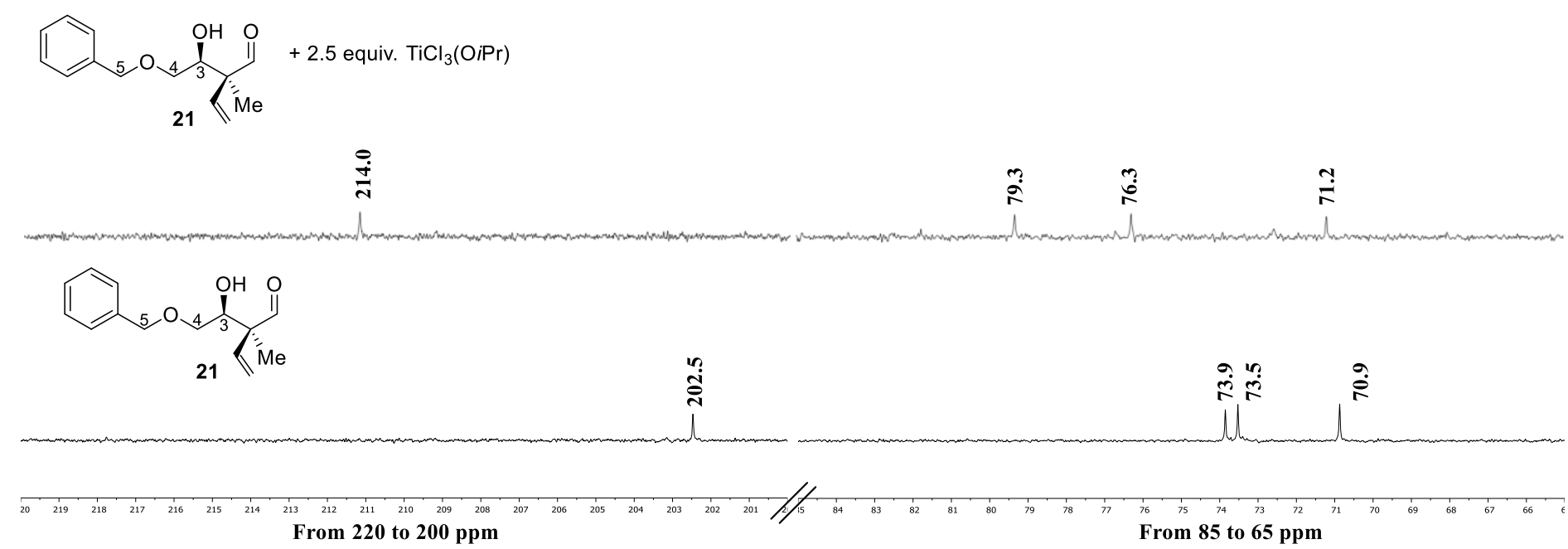

Figure 2. ${ }^{13} \mathrm{C}$ NMR spectra of aldehyde 21 with 0.0 and 2.5 equiv. of $\mathrm{TiCl}_{3}(\mathrm{OiPr})$ at $0{ }^{\circ} \mathrm{C}_{\text {in }} \mathrm{CD}_{2} \mathrm{Cl}_{2}$.

Although an X-ray structure of this titanium complex has yet to be determined, the above preliminary ${ }^{13} \mathrm{C}$ NMR spectra support formation of a complex as in intermediate $\mathbf{E}$ (Scheme 6). Formation of this intermediate is supported by Gau's studies of titanium complexes, ${ }^{33}$ where he noted the prevalence of hexacoordinated species. In addition, these studies suggested that the binding ability of various chemical entities to titanium was ${ }^{-} \mathrm{O} i \mathrm{Pr}>\mathrm{Cl}^{-}>\mathrm{THF}>\mathrm{Et}_{2} \mathrm{O}>\mathrm{PhCHO}>\mu-\mathrm{Cl}^{-}>\mathrm{RCO}_{2} \mathrm{M}$. Based on the ${ }^{13} \mathrm{C} \mathrm{NMR}$ data, the first step in the complexation of $\mathrm{TiCl}_{3}(\mathrm{O} i \mathrm{Pr})$ with aldehydes 19 and $\mathbf{2 1}$ is formation of a covalent bond between the oxygen at C3 and the titanium resulting in intermediate B (Scheme 6). Following Gau's study, the second oxygen to coordinate would be the oxygen at $\mathbf{C} 4$ resulting in intermediate $\mathbf{C}$ in which the carbonyl is uncoordinated. 

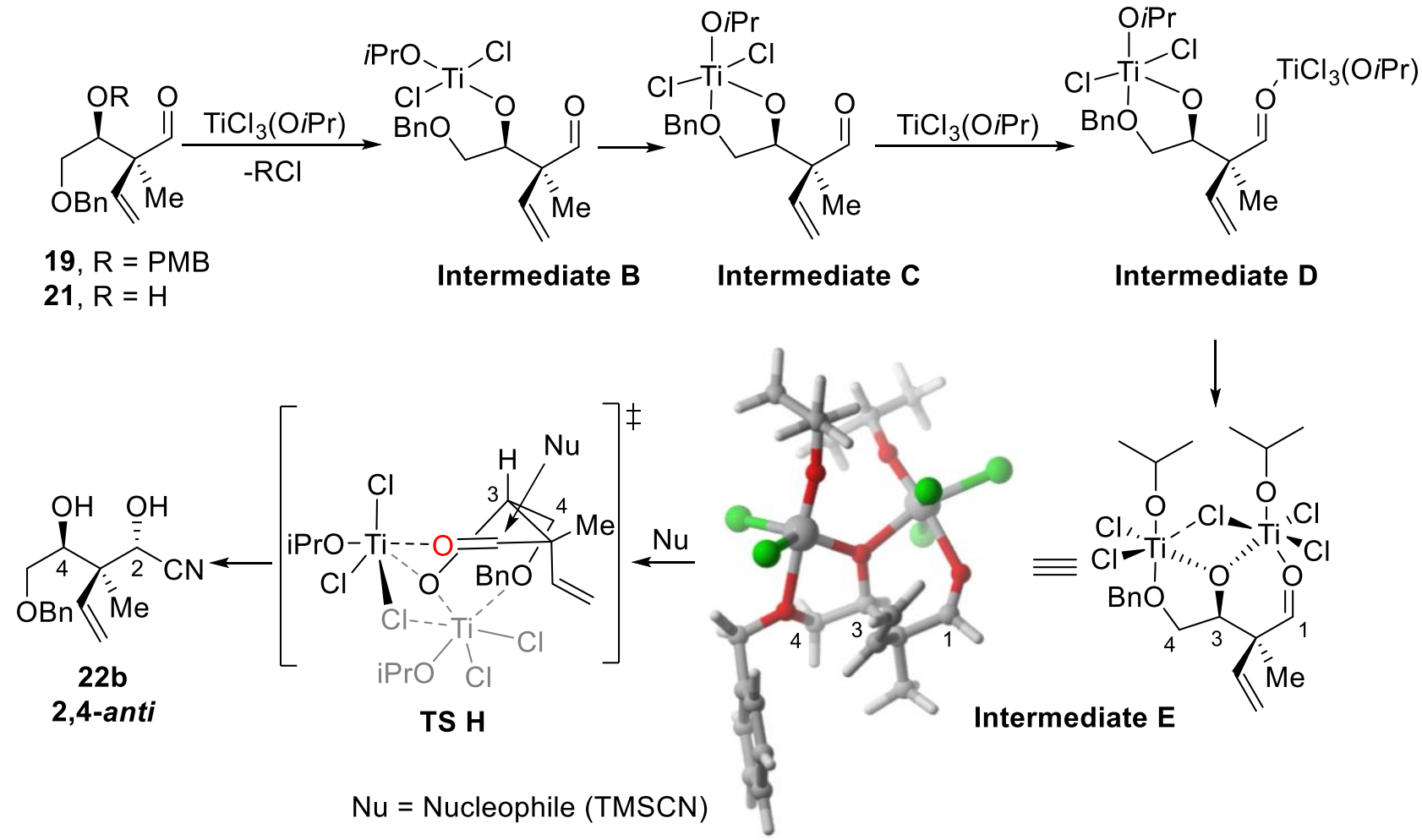

Scheme 6. Transition state for 2,4-anti cyanohydrin formation with 2.5 equivalents of $\mathrm{TiCl}_{3}(\mathrm{O} i \mathrm{Pr})$.

Although carbonyl activation is not necessary for cyanation to occur, ${ }^{13} \mathrm{C} N \mathrm{NM}$ studies indicate that it is indeed complexed with the titanium. Upon addition of a second equivalent of $\mathrm{TiCl}_{3}(\mathrm{OiPr})$, intermediate $\mathbf{D}$ could be formed, but monodentate activation is not likely to provide high diastereoselectivities, as observed with 1.1 equivalents of $\mathrm{TiCl}_{3}(\mathrm{O} \mathrm{Pr})$ (Table 1, entry 9). Formation of intermediate $\mathbf{E}$ in which both titaniums are coordinated to the oxygen at $\mathrm{C} 3$ would be consistent with the need for 2.5 equivalents of $\mathrm{TiCl}_{3}(\mathrm{OiPr})$ to reach higher levels of diastereoselectivity. Various titanium complexes were examined by density functional theory (DFT) calculations in Gaussian 09 (D.01) with tight SCF convergence ${ }^{34}$ using the M062X $35 / 6-31 G^{*}$ level of theory in DCM with the polarizable continuum model (PCM). ${ }^{36}$ Intermediate $E$ in which the isopropoxide ligands are located trans to the C1-aldehyde and C4-OBn group was of lowest energy. Through NBO analysis, a weak interaction was observed between the chloride ligand and the C4'-Ti thus both titanium centers exist as hexacoordinate species. Although, it is also possible to form a bicyclic [3.2.1]-type complex with 2.5 equivalents of $\mathrm{TiCl}_{3}(\mathrm{O} i \mathrm{Pr})$, as previously proposed, ${ }^{37}$ our preliminary ${ }^{13} \mathrm{C}$ NMR data is consistent with formation of intermediate $\mathbf{E}$ through initial displacement of a chloride ligand. Preferential attack of the cyanide opposite the $\beta$-alkyl chain in TS $\mathbf{H}$ would result in formation of the 2,4-anti cyanohydrin $\mathbf{2 2} \mathbf{b}$. Interestingly, when aldehyde 17 bearing a $\beta-\mathrm{OBn}$ group was reacted in the presence of 2.5 equivalents of $\mathrm{TiCl}_{3}(\mathrm{OiPr})$ (entry 13 , Table 1), a 1:1 ratio of syn and anti cyanohydrins $\mathbf{1 8 a , b}$ was obtained. The presence of this benzyl protecting group results in a reaction pathway not involving intermediate $\mathbf{E}$.

Investigation of this cyanation reaction has demonstrated that the choice of Lewis acid and $\beta$-protecting group are key to reach high levels of diastereoselectivity in favor of the 2,4-syn or 2,4-anti cyanohydrin allowing access to either the xylo-like (this manuscript) or the lyxo-like nucleoside analogues. In addition, this cyanation reaction highlights the potential for other stereoselective nucleophilic additions onto aldehydes possessing an $\alpha$-stereogenic center for which there are few literature examples. ${ }^{38,39}$ 
To generate the novel nucleoside scaffold, the C2-protected 2,4-syn cyanohydrin 16 was first reduced with DIBAL-H and then transformed into di(ethylthio)acetal 4 in $70 \%$ yield (Scheme 7).

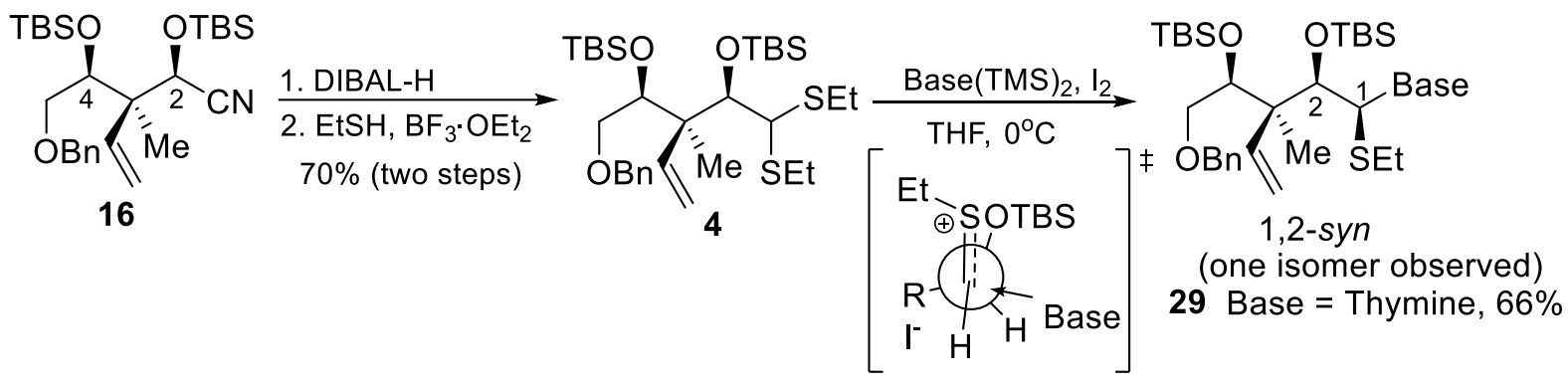

TS I
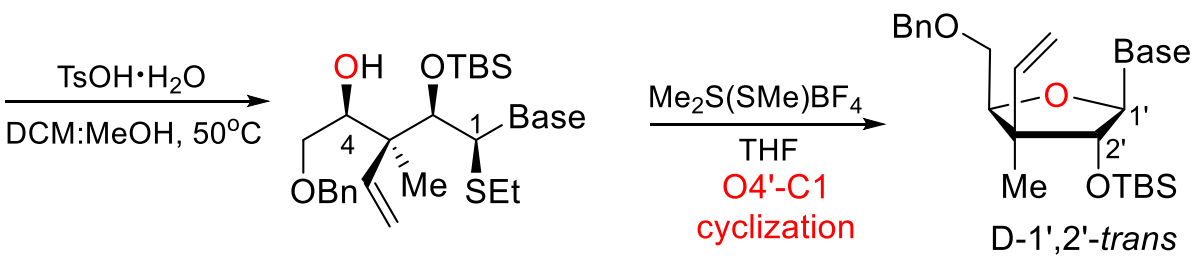

$$
\begin{aligned}
30 & \text { Base }= \\
31 \text { Base }= & \text { Thymine }, 73 \% \\
& \text { (two steps) }
\end{aligned}
$$

32, Base $=$ Thymine, $71 \%$

33, Base $=$ Uracil, $80 \%$

Scheme 7. Acyclic approach for the synthesis of nucleoside analogues.

At this point in the strategy, we made use of our novel two-step acyclic approach for the synthesis of nucleoside analogues, ${ }^{40}$ the mechanism of which has been examined in detail using DFT calculations. ${ }^{8,41}$ We have recently reported the use of this strategy with a C3-quaternary center and a fluoride at $\mathrm{C} 2,{ }^{8}$ however, this is the first report of using this strategy with a thioaminal bearing a C2-hydroxy and a C3-quaternary center. An essential aspect of this strategy is the diastereoselective synthesis of a 1,2-syn thioaminal (29) from a dithioacetal (4). Activation of this acyclic dithioacetal with iodine and addition of silylated nucleobase (thymine or uracil) provided the 1,2-syn thioaminals in excellent diastereoselectivity (only one isomer could be detected by ${ }^{1} \mathrm{H}$ NMR) and yield. Formation of the 1,2-syn thioaminal proceeds through a $\mathrm{S}_{\mathrm{N}} 2$-like mechanism in which the initially formed halothioether adopts a conformational preference orienting the C2-OTBS and the thioether moiety in close proximity to one another to maximize $\mathrm{R}-\mathrm{C} 2$ and $\mathrm{H}-\mathrm{C} 2$ sigma donation to the electron poor thiacarbenium intermediate in TS I. The presence of the counteranion ( $\left.I^{-}\right)$stabilizes this transition state and prefers to be located on the opposite side of the incoming nucleobase. ${ }^{8,41}$ Selective removal of the less hindered C4-TBS protecting group provided the necessary thioaminals $\mathbf{3 0}$ and $\mathbf{3 1}$ in excellent yields. The next key step of our acyclic approach involves a stereospecific displacement of the activated sulfur of the thioaminal by the C4-hydroxy with inversion of configuration ( $\mathrm{O}^{\prime}$ '-C1 cyclization). This provided the D-1',2'-trans furanosides 32 (thymine, 71\% yield) and $\mathbf{3 3}$ (uracil, $80 \%$ yield). Proof of structure for the nucleoside analogues was determined by 2D NOESY experiments (see supporting information). Transformation of the monosubstituted alkene of $\mathbf{3 2}$ and $\mathbf{3 3}$ into the desired primary alcohol was accomplished in five steps (Scheme 8). This monosubstituted alkene could serve as the starting point for a variety of chemical modifications to add heteroatoms and diverse functionalities. In this study, it was transformed into an hydroxymethyl group. 


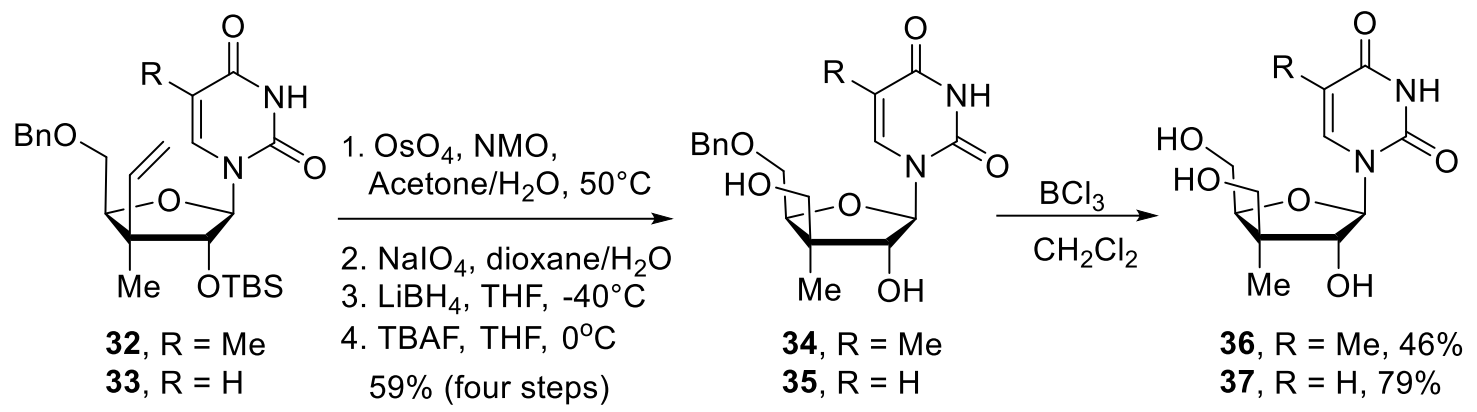

Scheme 8. Synthesis of xylo-like nucleoside analogues.

Dihydroxylation using osmium tetroxide followed by oxidative cleavage of the diol resulted in the corresponding aldehyde that was reduced using lithium borohydride providing the C3'-hydroxymethyl substituent. Removal of the C2'-TBS provided scaffolds 34 and 35 in 59\% yield for these four steps. The desired xylo-like nucleoside analogues (36 and 37) were formed after removal of the primary benzyl protecting groups.

\section{Conclusions}

The synthesis of a novel class of xylo-like nucleoside analogues bearing a stereogenic quaternary center at C3' has been described. This acyclic synthetic route relies on highly diastereoselective chemical transformations investigated in our laboratory: an anti selective Mukaiyama aldol reaction, a syn selective intramolecular radical transfer cyclization, formation of a syn selective cyanohydrin followed by the synthesis and cyclization of a syn thioaminal. A key element of this route is the cyanide addition onto aldehydes activated through formation of proposed 7-membered ring chelates. These analogues and other molecules of this family are being tested for their antiviral and antiproliferative properties. The results of these tests will be reported in due course.

\section{Experimental Section}

General Comments. All reactions requiring anhydrous conditions were carried out under an atmosphere of nitrogen or argon in flame-dried glassware using standard syringe techniques. All anhydrous solvents were dried with $4 \AA$ molecular sieves prior to use. The $4 \AA$ molecular sieves (1-2 mm beads) were activated by heating at $180{ }^{\circ} \mathrm{C}$ for 48 hours under vacuum prior to adding to new bottles of solvent purged with argon. Commercially available reagents were used as received. Flash chromatography was performed on silica gel 60 $(0.040-0.063 \mathrm{~mm})$ using forced flow (flash chromatography) of the indicated solvent system or an automated flash purification system Biotage Isolera One (version 1.3.6). Analytical thin-layer chromatography (TLC) was carried out on pre-coated $(0.25 \mathrm{~mm})$ silica gel aluminum plates. Visualization was performed with U.V. short wavelength and/or revealed with ammonium molybdate or potassium permanganate solutions. ${ }^{1} \mathrm{H}$ NMR spectra were recorded at room temperature on a $500 \mathrm{MHz}$ Varian Unity INOVA NMR spectrometer. The data are reported as follows: chemical shift in ppm referenced to residual solvent $\left(\mathrm{CDCl}_{3} \delta 7.26 \mathrm{ppm}\right)$, multiplicity (s $=$ singlet,$d=$ doublet,$d d=$ doublet of doublets, $d d d=$ doublet of doublets of doublets, $t=$ triplet, $t d=$ triplet of 
doublets, $\mathrm{m}=$ multiplet, app = apparent), coupling constants $(\mathrm{Hz})$, and integration. ${ }^{13} \mathrm{C}$ NMR spectra were recorded at room temperature using 100.6 (Varian VXR $400 \mathrm{NMR}$ ) or $126 \mathrm{MHz}$. The data are reported as follows: chemical shift in ppm referenced to residual solvent $\left(\mathrm{CDCl}_{3} \delta 77.16 \mathrm{ppm}\right)$. Infrared spectra were recorded on a FTIR ABB Bomen (MB series) or a Bruker Platinum ATR (Alpha II series) spectrophotometer from a thin film of purified product and signals are reported in $\mathrm{cm}^{-1}$. Mass spectra were recorded through electrospray ionization (ESI) positive ion mode using a Thermo Fischer LTQ Orbitrap XL. A Q exactive mass analyzer was used for HRMS measurements and were done by the Plateforme de découvertes en protéomique at I'Institut de Recherches Cliniques de Montréal (IRCM). Optical rotations were measured at room temperature from the sodium $\mathrm{D}$ line $(589 \mathrm{~nm})$ using a PerkinElmer 343 polarimeter and $\mathrm{CDCl}_{3}$ as solvent unless otherwise noted and calculated using the formula: $[\alpha]_{D}=(100) \alpha_{\mathrm{obs}} /(\ell \cdot(c))$, where $c=(\mathrm{g}$ of substrate/100 $\mathrm{ml}$ of solvent) and $l=1 \mathrm{dm}$. The characterization of chemical structures from $X$-ray data has been done at the Université de Montréal X-ray diffraction laboratory. Proofs of structure can be found in the supporting information.

\section{General Procedure A: Reduction of ester}

To a solution of ester in dry $\mathrm{CH}_{2} \mathrm{Cl}_{2}(67 \mathrm{~mL}, 0.20 \mathrm{M})$ at $-40^{\circ} \mathrm{C}$, DIBAL-H (3.0 equiv.) was added. The solution was stirred until the ester (1.0 equiv.) was all consumed ( $1 \mathrm{~h} 30$ at $-40{ }^{\circ} \mathrm{C}$ ) as determined by TLC. MeOH was added at $-40^{\circ} \mathrm{C}$ until gas formation ceased. An aqueous solution of potassium sodium tartrate was added, and the reaction mixture was warmed to room temperature. The aqueous layer was extracted with $\mathrm{Et}_{2} \mathrm{O}(3 \mathrm{x})$. The organic layers were combined, dried over $\mathrm{MgSO}_{4}$, filtered and concentrated in vacuo.

\section{General Procedure B: Oxidation of alcohol}

To a solution of oxalyl chloride (1.3 equiv.) in anhydrous $\mathrm{CH}_{2} \mathrm{Cl}_{2}(0.10 \mathrm{M})$ at $-78{ }^{\circ} \mathrm{C}$, dimethyl sulfoxide (2.3 equiv.) was added dropwise. The solution was stirred for 20 minutes at which point the alcohol (1.0 equiv.), as a $0.40 \mathrm{M}$ solution in anhydrous $\mathrm{CH}_{2} \mathrm{Cl}_{2}$, was added followed by stirring at $-78^{\circ} \mathrm{C}$ for $1 \mathrm{~h}$. Triethylamine (5.0 equiv.) was added and the reaction mixture was warmed to room temperature over 45 minutes. An aqueous solution of $\mathrm{NH}_{4} \mathrm{Cl}$ was added and the aqueous layer was extracted with $\mathrm{Et}_{2} \mathrm{O}(3 \mathrm{x})$. The organic layers were combined, dried over $\mathrm{MgSO}_{4}$, filtered and concentrated in vacuo.

\section{General Procedure C: Cyanohydrin formation}

To a solution of aldehyde (1.0 equiv.) in anhydrous $\mathrm{CH}_{2} \mathrm{Cl}_{2}(0.10 \mathrm{M})$ at $0{ }^{\circ} \mathrm{C}$, the appropriate Lewis acid, $\mathrm{MgBr}_{2} \cdot \mathrm{OEt}_{2}$ (5.0 equiv.), $\mathrm{BF}_{3} \cdot \mathrm{OEt}_{2}$ (1.5 equiv.), or $\mathrm{TiCl}_{3}(\mathrm{O} i \mathrm{Pr})$ (1.1 or 2.5 equiv.), was added. The reaction mixture was stirred 5 minutes for precomplexation. TMSCN (2.0 equiv.) was then added. The solution was stirred until the aldehyde was all consumed, $1 \mathrm{~h}$ at $0{ }^{\circ} \mathrm{C}$, as determined by TLC. An aqueous solution of $\mathrm{NaHCO}_{3}$ was added and the aqueous layer was extracted with $\mathrm{Et}_{2} \mathrm{O}(3 \mathrm{x})$. The organic layers were combined, dried over $\mathrm{MgSO}_{4}$, filtered and concentrated in vacuo.

$\mathrm{TiCl}_{3}(\mathrm{OiPr})$ was prepared by mixing one equivalent of $\mathrm{Ti}(\mathrm{O} i \mathrm{Pr})_{4}$ with three equivalents of $\mathrm{TiCl}_{4}$ in $\mathrm{DCM} .{ }^{42}$

\section{Preparation of silylated nucleobases}

To a suspension of the nucleobase in HMDS (2.7 equiv.) under inert atmosphere, $\left(\mathrm{NH}_{4}\right)_{2} \mathrm{SO}_{4}(0.10$ equiv.) was added. The reaction mixture was refluxed until a clear solution was obtained (typically three hours). Upon cooling to room temperature, the solution was placed under high vacuum for approximately 1 hour to remove excess HMDS. A solution of the silylated nucleobase was made in dichloroethane.

\section{Compounds 8a,b and 12 from Scheme 2:}

(-)-Methyl (2R,3R)-2-bromo-3-\{[dimethyl(vinyl)silyl]oxy\}-3-((R)-2,2-dimethyl-1,3-dioxolan-4-yl)-2-methylpropanoate (8a) and (+)-methyl (2S,3R)-2-bromo-3-\{[dimethyl(vinyl)silyl]oxy\}-3-((R)-2,2-dimethyl-1,3-dioxolan4-yl)-2-methylpropanoate (8b): To a $0{ }^{\circ} \mathrm{C}$ solution of crude methyl (3R)-2-bromo-3-((R)-2,2-dimethyl-1,3dioxolan-4-yl)-3-hydroxy-2-methylpropanoate ${ }^{7}\left(4.8 \mathrm{~g}, 16 \mathrm{mmol}, 1.0\right.$ equiv.) in $\mathrm{CH}_{2} \mathrm{Cl}_{2}(40 \mathrm{~mL}, 0.40 \mathrm{M})$, 
imidazole ( $3.3 \mathrm{~g}, 48 \mathrm{mmol}, 3.0$ equiv.) was added in one portion. Upon complete dissolution of imidazole, chloro(dimethyl)vinylsilane $(3.14 \mathrm{~mL}, 20.8 \mathrm{mmol}, 1.30$ equiv.) was added dropwise. The reaction was then warmed to room temperature and stirring continued for 16 hours at which point ${ }^{1} \mathrm{H}$ NMR showed complete conversion of starting material. The reaction mixture was quenched with water. The aqueous layer was extracted with $\mathrm{CH}_{2} \mathrm{Cl}_{2}(3 \times 25 \mathrm{~mL})$, the organic layers were combined, washed with brine, dried over $\mathrm{MgSO}_{4}$, filtered and concentrated in vacuo to afford the crude product as a dark yellow oil. Coevaporation with toluene $\left(2 \mathrm{x}\right.$ ) and purification using a silica pad (Hexanes/Et $\left.{ }_{2} \mathrm{O}, 30: 70\right)$ provided $8 \mathbf{a}, \mathbf{b}$ (3.2 g, 52\% yield over 3 steps, 3,4-anti : 3,4-syn 10:1) as a light-yellow oil. The minor product from the aldol reaction (11a,b) could not be separated. 8a: $\mathrm{R}_{f}=0.37$ (Hexanes/Et ${ }_{2} \mathrm{O} 90: 10$ ); $[\alpha]_{\mathrm{D}}^{25}-2.7\left(c=2.0, \mathrm{CH}_{2} \mathrm{Cl}_{2}\right.$ ); Molecular formula: $\mathrm{C}_{14} \mathrm{H}_{25} \mathrm{BrO}_{5} \mathrm{Si}$; MW: 381.34; IR (neat) $v_{\max } 2985,2953,1744,1250,1053 \mathrm{~cm}^{-1} ;{ }^{1} \mathrm{H} \mathrm{NMR}\left(500 \mathrm{MHz}, \mathrm{CDCl}_{3}\right) \delta 6.15$ (dd, J 20.3, $14.9 \mathrm{~Hz}, 1 \mathrm{H}), 6.02$ (dd, J 14.9, 3.9 Hz, 1H), $5.75(\mathrm{dd}, J 20.3,3.8 \mathrm{~Hz}, 1 \mathrm{H}), 4.62-4.57(\mathrm{~m}, 2 \mathrm{H}), 3.98-3.91(\mathrm{~m}, 2 \mathrm{H})$, $3.78(\mathrm{~s}, 3 \mathrm{H}), 1.77(\mathrm{~s}, 3 \mathrm{H}), 1.46(\mathrm{~s}, 3 \mathrm{H}), 1.35(\mathrm{~s}, 3 \mathrm{H}), 0.24(\mathrm{~s}, 3 \mathrm{H}), 0.23(\mathrm{~s}, 3 \mathrm{H}) \mathrm{ppm} ;{ }^{13} \mathrm{C} \mathrm{NMR}(126 \mathrm{MHz} \mathrm{CDCl}) \delta$ 170.6, 137.7, 133.4, 108.1, 76.9, 75.9, 64.6, 61.4, 53.4, 26.2, 24.5, 23.8, -0.9, -1.3 ppm; HRMS calcd for $\mathrm{C}_{14} \mathrm{H}_{25} \mathrm{BrO}_{5} \mathrm{SiNa}\left[\mathrm{M}+\mathrm{Na}^{+}\right]:$403.0547; found 403.0533 (-3.5 ppm). 8b (6:1 mix with 3,4-syn product): $\mathrm{R}_{f}=0.21$ (Hexanes/Et $\left.{ }_{2} \mathrm{O} 90: 10\right) ;[\alpha]_{\mathrm{D}}^{25}+11\left(c=0.8, \mathrm{CH}_{2} \mathrm{Cl}_{2}\right)$; IR (neat) $v_{\max } 2987,2952,1744,1254,1104 \mathrm{~cm}^{-1} ;{ }^{1} \mathrm{H} \mathrm{NMR}(500$ $\left.\mathrm{MHz}, \mathrm{CDCl}_{3}\right) \delta 6.25$ (dd, J 20.4, $\left.14.9 \mathrm{~Hz}, 1 \mathrm{H}\right), 6.07$ (dd, J 14.9, 3.7 Hz, 1H), 5.82 (dd, J 20.4, 3.7 Hz, $\left.1 \mathrm{H}\right), 4.33(\mathrm{~d}, J$ $7.2 \mathrm{~Hz}, 1 \mathrm{H}), 4.09$ (dd, J 8.1, $6.5 \mathrm{~Hz}, 1 \mathrm{H}), 4.02$ (dd, J 13.5, $6.5 \mathrm{~Hz}, 1 \mathrm{H}), 3.81-3.79(\mathrm{~m}, 1 \mathrm{H}), 3.76(\mathrm{~s}, 3 \mathrm{H}), 1.89(\mathrm{~s}$, $3 \mathrm{H}), 1.35(\mathrm{~s}, 3 \mathrm{H}), 1.29(\mathrm{~s}, 3 \mathrm{H}), 0.34(\mathrm{~s}, 3 \mathrm{H}), 0.31(\mathrm{~s}, 3 \mathrm{H}) \mathrm{ppm} ;{ }^{13} \mathrm{C} \mathrm{NMR}\left(126 \mathrm{MHz}, \mathrm{CDCl}_{3}\right) \delta$ 170.8, 137.7, 133.7, 109.8, 78.4, 76.4, 68.3, 64.9, 52.9, 26.1, 25.1, 22.2, -0.7, -1.0 ppm; HRMS calcd for $\mathrm{C}_{14} \mathrm{H}_{25} \mathrm{BrO}_{5} \mathrm{SiNa}\left[\mathrm{M}+\mathrm{Na}^{+}\right]$: 403.0547; found 403.0533 (-3.5 ppm).

(-)-Methyl (S)-2-((S)-[(R)-2,2-dimethyl-1,3-dioxolan-4-yl)(hydroxy)methyl]-2-methylbut-3-enoate (12): To a solution of the $\alpha$-bromo ester $8 \mathbf{a}, \mathbf{b}\left(2.0 \mathrm{~g}, 5.2 \mathrm{mmol}, 1.0\right.$ equiv.) in toluene $(11 \mathrm{~mL}, 0.50 \mathrm{M})$ at $0{ }^{\circ} \mathrm{C}, \mathrm{BEt}_{3}(5.2$ $\mathrm{mL}, 1.0 \mathrm{M}, 1.0$ equiv.) was added with a syringe pump over 4 hours. After completion of the reaction, ethanolamine $(0.90 \mathrm{~mL}, 16 \mathrm{mmol}, 3.0$ equiv.) was added. The mixture was stirred for 30 minutes before it was transferred to a plastic vial and treated with $3 \mathrm{HF} \cdot \mathrm{NEt}_{3}(1.3 \mathrm{~mL}, 7.9 \mathrm{mmol}, 1.5$ equiv. $)$ at $0{ }^{\circ} \mathrm{C}$. The mixture was stirred at room temperature overnight. The crude solution was transferred dropwise into a cold solution of saturated $\mathrm{NaHCO}_{3}$. The aqueous layer was extracted with $\mathrm{Et}_{2} \mathrm{O}(2 \mathrm{x})$, the organic layers were combined, dried over $\mathrm{MgSO}_{4}$, filtered and concentrated in vacuo. The crude product (2,3-syn, dr >20:1) was purified by flash chromatography on silica gel (Hexanes/Et $2 \mathrm{O}, 60: 40)$ to give 12 (1.05 g, 82\%) as a colorless oil. The minor 3,4syn product from the Mukaiyama aldol (not characterized) could be separated. 12: $\mathrm{R}_{f}=0.3$ (Hexanes/EtOAc, 65:35); $[\alpha]_{\mathrm{D}}^{25}-20\left(c=1.1, \mathrm{CH}_{2} \mathrm{Cl}_{2}\right)$; Molecular formula: $\mathrm{C}_{12} \mathrm{H}_{20} \mathrm{O}_{5} ; \mathrm{MW}: 244.29 ; \mathrm{IR}$ (neat) $\mathrm{v}_{\max } 1722 \mathrm{~cm}^{-1} ;{ }^{1} \mathrm{H} \mathrm{NMR}$ $\left(500 \mathrm{MHz}, \mathrm{CDCl}_{3}\right) \delta 6.12$ (dd, J 17.6, $\left.10.8 \mathrm{~Hz}, 1 \mathrm{H}\right), 5.32$ (d, J $\left.10.8 \mathrm{~Hz}, 1 \mathrm{H}\right), 5.25$ (d, J $\left.17.6 \mathrm{~Hz}, 1 \mathrm{H}\right), 4.08-3.99(\mathrm{~m}$, $3 \mathrm{H}), 3.96-3.91(\mathrm{~m}, 1 \mathrm{H}), 3.70(\mathrm{~s}, 3 \mathrm{H}), 1.37(\mathrm{~s}, 3 \mathrm{H}), 1.35(\mathrm{~s}, 3 \mathrm{H}), 1.32(\mathrm{~s}, 3 \mathrm{H}) \mathrm{ppm}, \mathrm{OH}$ signal missing possibly due to exchange in $\mathrm{CDCl}_{3} ;{ }^{13} \mathrm{C} \mathrm{NMR}\left(126 \mathrm{MHz} \mathrm{CDCl}_{3}\right) \delta 175.0,138.4,117.3,109.1,76.1,75.6,67.2,52.8,52.4,26.4$, 25.4, 15.2 ppm; HRMS calcd for: $\mathrm{C}_{12} \mathrm{H}_{20} \mathrm{O}_{5} \mathrm{Na}\left[\mathrm{M}+\mathrm{Na}^{+}\right]$: 267.1203; found 267.1204 (+0.4 ppm).

\section{Compounds 13, 14, 15 and 6 from Scheme 3:}

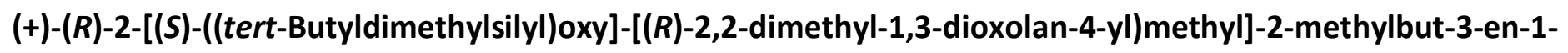
yl benzoate (13): To a solution of secondary alcohol 12 (4.9 g, $20 \mathrm{mmol}, 1.0$ equiv.) in $\mathrm{CH}_{2} \mathrm{Cl}_{2}(25 \mathrm{~mL}, 0.80 \mathrm{M})$ at $0{ }^{\circ} \mathrm{C}$ pyridine $(3.2 \mathrm{~mL}, 40 \mathrm{mmol}, 2.0$ equiv.) and TBSOTf (5.5 mL, $24 \mathrm{mmol}, 1.2$ equiv.) were added. The resulting mixture was stirred at room temperature for 16 hours before addition of $\mathrm{Et}_{2} \mathrm{O} / \mathrm{Hexanes}(1: 1,25 \mathrm{~mL})$ and saturated aqueous $\mathrm{NH}_{4} \mathrm{Cl}(30 \mathrm{~mL})$. After stirring vigorously for 10 minutes, the layers were separated, and the aqueous layer extracted with $\mathrm{Et}_{2} \mathrm{O} /$ Hexanes $(1: 1,2 \times 30 \mathrm{~mL})$. The organic fractions were combined and washed successively with $1 \mathrm{~N} \mathrm{HCl}(2 \times 40 \mathrm{~mL})$, water and brine, dried over $\mathrm{MgSO}_{4}$, filtered and concentrated in vacuo. The crude residue $(7.2 \mathrm{~g}$, 99\% yield) was coevaporated with toluene $(2 \times 10 \mathrm{~mL})$ and used directly in the 
next reaction. A solution of the crude ester $\left(7.2 \mathrm{~g}, 20 \mathrm{mmol}, 1.0\right.$ equiv.) in $\mathrm{CH}_{2} \mathrm{Cl}_{2}(40 \mathrm{~mL}, 0.50 \mathrm{M})$ was cooled to $-40^{\circ} \mathrm{C}$ (MeCN/dry ice bath) followed by addition of DIBAL-H (45 mL, $45 \mathrm{mmol}, 2.2$ equiv.) over 10 minutes. After stirring 90 minutes at $-40^{\circ} \mathrm{C}$, a saturated solution of aqueous Rochelle salt $(60 \mathrm{~mL})$ was slowly added followed by saturated aqueous $\mathrm{Na}_{2} \mathrm{CO}_{3}(10 \mathrm{~mL})$ and $\mathrm{Et}_{2} \mathrm{O}(40 \mathrm{~mL})$. The biphasic mixture was warmed to room temperature and vigorously stirred until both phases were clear (about 2 hours). The aqueous layer was extracted with $\mathrm{Et}_{2} \mathrm{O}(2 \times 40 \mathrm{~mL})$ and the combined organic fractions were washed with saturated aqueous $\mathrm{Na}_{2} \mathrm{CO}_{3}$ followed by brine, dried over $\mathrm{MgSO}_{4}$, filtered and concentrated in vacuo to afford the crude primary alcohol as a colorless oil which was used directly in the next step. The crude primary alcohol (6.6 g, $20 \mathrm{mmol}$, 1.0 equiv.) was dissolved in $\mathrm{CH}_{2} \mathrm{Cl}_{2}(20 \mathrm{~mL}, 1.0 \mathrm{M})$ and pyridine $(6.5 \mathrm{~mL}, 80 \mathrm{mmol}, 4.0$ equiv.) and DMAP (0.49 $\mathrm{g}, 4.0 \mathrm{mmol}, 0.20$ equiv.) were added. The resulting reaction mixture was cooled to $0{ }^{\circ} \mathrm{C}$ and benzoyl chloride (2.8 $\mathrm{mL}, 24 \mathrm{mmol}, 1.2$ equiv.) was added dropwise. The reaction mixture was slowly warmed to room temperature and stirred for 5 hours before being cooled to $0{ }^{\circ} \mathrm{C}$ followed by addition of ethylenediamine $(0.94$ $\mathrm{mL}, 14 \mathrm{mmol}, 0.70$ equiv.). After stirring 45 minutes, $\mathrm{CH}_{2} \mathrm{Cl}_{2}$ was evaporated and the residue diluted with $\mathrm{Et}_{2} \mathrm{O}$, loaded on a silica pad and eluted with Hexanes/EtOAc (1:1) to afford the crude product as an oil. Purification by flash column chromatography provided 13 as a colorless oil (5.9 g, 68\% yield over 3 steps). $R_{f}=0.68$ (Hexanes/EtOAc, 80:20); $[\alpha]_{\mathrm{D}}^{25}+24\left(c=2.6, \mathrm{CH}_{2} \mathrm{Cl}_{2}\right)$; Molecular formula: $\mathrm{C}_{24} \mathrm{H}_{38} \mathrm{O}_{5} \mathrm{Si}$; MW: 434.25; IR (neat) $v_{\max }$ $1722 \mathrm{~cm}^{-1} ;{ }^{1} \mathrm{H}$ NMR $\left(500 \mathrm{MHz}, \mathrm{CDCl}_{3}\right) \delta 8.08-8.02(\mathrm{~m}, 2 \mathrm{H}), 7.61-7.55(\mathrm{~m}, 1 \mathrm{H}), 7.50-7.43(\mathrm{~m}, 2 \mathrm{H}), 5.99(\mathrm{dd}, J$ $17.7,11.0 \mathrm{~Hz}, 1 \mathrm{H}), 5.26-5.18(\mathrm{~m}, 2 \mathrm{H}), 4.27-4.20(\mathrm{~m}, 4 \mathrm{H}), 3.81(\mathrm{~d}, J 7.5 \mathrm{~Hz}, 2 \mathrm{H}), 1.45(\mathrm{~s}, 3 \mathrm{H}), 1.32(\mathrm{~s}, 3 \mathrm{H}), 1.22$ $(\mathrm{s}, 3 \mathrm{H}), 0.93(\mathrm{~s}, 9 \mathrm{H}), 0.16(\mathrm{~s}, 3 \mathrm{H}), 0.07(\mathrm{~s}, 3 \mathrm{H}) \mathrm{ppm} ;{ }^{13} \mathrm{C} \mathrm{NMR}\left(126 \mathrm{MHz}, \mathrm{CDCl}_{3}\right) \delta 166.5,139.0,133.1,130.4$, 129.6, 128.6, 116.4, 110.1, 107.4, 76.2, 75.3, 69.4, 64.4, 45.1, 26.3, 24.9, 18.9, 18.6, -3.0, -4.4 ppm; HRMS calcd for: $\mathrm{C}_{24} \mathrm{H}_{38} \mathrm{O}_{5} \mathrm{SiNa}\left[\mathrm{M}+\mathrm{Na}^{+}\right]$: 457.2381 ; found 457.2374 (-1.5 ppm).

$(+)-(R)-2-\left\{(S)-1-\left[\left(\right.\right.\right.$ tert-Butyldimethylsilyl)oxy]-2-hydroxyethyl\}-2-methylbut-3-en-1-yl benzoate (14): To a $0{ }^{\circ} \mathrm{C}$ solution of acetal 13 (5.2 g, $12 \mathrm{mmol}, 1.0$ equiv.) in EtOAc (120 mL, $0.10 \mathrm{M})$, periodic acid (4.1 g, $18 \mathrm{mmol}, 1.5$ equiv.) was added in one portion. The resulting heterogeneous mixture was stirred vigorously for 15 minutes at $0{ }^{\circ} \mathrm{C}$ and then at room temperature until complete conversion (typically 3-4 hours by TLC monitoring). The mixture was diluted with $\mathrm{Et}_{2} \mathrm{O}(100 \mathrm{~mL})$ and slowly poured into cold saturated aqueous $\mathrm{Na}_{2} \mathrm{~S}_{2} \mathrm{O}_{3}(75 \mathrm{~mL})$ and saturated aqueous $\mathrm{Na}_{2} \mathrm{CO}_{3}(10 \mathrm{~mL})$. After vigorously stirring 30 minutes, the aqueous layer was extracted with $\mathrm{Et}_{2} \mathrm{O}(1 \times 50 \mathrm{~mL})$ and the combined organic fractions were washed with brine, dried over $\mathrm{MgSO}_{4}$, filtered and concentrated in vacuo to afford the aldehyde $(4.4 \mathrm{~g}, 100 \%$ yield, crude) which was used directly in the next step. ${ }^{1} \mathrm{H}$ NMR $\left(500 \mathrm{MHz}, \mathrm{CDCl}_{3}\right) \delta 9.59(\mathrm{~d}, J 3.1 \mathrm{~Hz}, 1 \mathrm{H}), 8.04-7.99(\mathrm{~m}, 2 \mathrm{H}), 7.63-7.56(\mathrm{~m}, 1 \mathrm{H}), 7.52-7.43(\mathrm{~m}$, $2 \mathrm{H}), 6.10(\mathrm{dd}, J 17.7,11.0 \mathrm{~Hz}, 1 \mathrm{H}), 5.29(\mathrm{~d}, J 11.0 \mathrm{~Hz}, 1 \mathrm{H}), 5.24(\mathrm{dd}, J 17.6,0.7 \mathrm{~Hz}, 1 \mathrm{H}), 4.35(\mathrm{~d}, J 10.8 \mathrm{~Hz}, 1 \mathrm{H})$, $4.23(\mathrm{~d}, J 10.8 \mathrm{~Hz}, 1 \mathrm{H}), 3.94(\mathrm{~d}, J 3.1 \mathrm{~Hz}, 1 \mathrm{H}), 1.24(\mathrm{~s}, 3 \mathrm{H}), 0.94(\mathrm{~s}, 9 \mathrm{H}), 0.05(\mathrm{~s}, 3 \mathrm{H}), 0.04(\mathrm{~s}, 3 \mathrm{H}) \mathrm{ppm}$ ). The crude aldehyde ( $4.4 \mathrm{~g}, 12 \mathrm{mmol}, 1.0$ equiv.) was dissolved in THF $(60 \mathrm{~mL}, 0.20 \mathrm{M})$ and the resulting solution was cooled to $-40^{\circ} \mathrm{C}$. $\mathrm{LiBH}_{4}(2.0 \mathrm{M}$ in THF, $6.0 \mathrm{~mL}, 12 \mathrm{mmol}, 1.0$ equiv.) was then added dropwise and the reaction mixture stirred at $-40^{\circ} \mathrm{C}$ for 1 hour. The reaction was quenched by slow addition of $0.1 \mathrm{~N} \mathrm{HCl}(60 \mathrm{~mL})$ and the biphasic mixture stirred vigorously for 30 minutes at room temperature at which point $\mathrm{Et}_{2} \mathrm{O}$ was added. The aqueous layer was extracted with $\mathrm{Et}_{2} \mathrm{O}(2 \times 30 \mathrm{~mL})$ and the combined organic layers washed with $1 \mathrm{~N} \mathrm{HCl}(25$ $\mathrm{mL}$ ), water and brine, dried over $\mathrm{MgSO}_{4}$, filtered and concentrated in vacuo to afford the crude primary alcohol. Purification by flash chromatography provided 14 as a colorless oil ( $3.7 \mathrm{~g}, 85 \%$ yield over 2 steps): $\mathrm{R}_{f}=$ 0.37 (Hexanes/EtOAc, 80:20); $[\alpha]_{\mathrm{D}}^{25}+4.8\left(c=6.3, \mathrm{CH}_{2} \mathrm{Cl}_{2}\right)$; Molecular formula: $\mathrm{C}_{20} \mathrm{H}_{32} \mathrm{O}_{4} \mathrm{Si}$; MW: 364.56; IR (neat) $V_{\max } 3501,1720,1704 \mathrm{~cm}^{-1} ;{ }^{1} \mathrm{H}$ NMR $\left(500 \mathrm{MHz}, \mathrm{CDCl}_{3}\right) \delta 8.08-8.03(\mathrm{~m}, 2 \mathrm{H}), 7.62-7.55(\mathrm{~m}, 1 \mathrm{H}), 7.50-7.43$ $(\mathrm{m}, 2 \mathrm{H}), 6.07(\mathrm{dd}, J 17.7,11.0 \mathrm{~Hz}, 1 \mathrm{H}), 5.27-5.16(\mathrm{~m}, 2 \mathrm{H}), 4.30(\mathrm{~d}, J 10.8 \mathrm{~Hz}, 1 \mathrm{H}), 4.27(\mathrm{~d}, J 10.8 \mathrm{~Hz}, 1 \mathrm{H}), 3.85$ (dd, J 5.3, 3.5 Hz, 1H), 3.75 (ddd, J 11.5, 5.7, $3.6 \mathrm{~Hz}, 1 \mathrm{H}), 3.71-3.64(\mathrm{~m}, 1 \mathrm{H}), 1.21(\mathrm{~s}, 3 \mathrm{H}), 0.94(\mathrm{~s}, 9 \mathrm{H}), 0.15(\mathrm{~s}$,

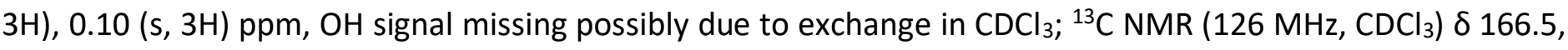


139.8, 133.1, 130.4, 129.6, 128.6, 115.7, 76.9, 69.0, 64.3, 44.9, 26.1, 19.0, 18.4, -3.8, -4.7 ppm; HRMS calcd for $\mathrm{C}_{20} \mathrm{H}_{33} \mathrm{O}_{4} \mathrm{Si}\left[\mathrm{M}+\mathrm{H}^{+}\right]: 365.2143$; found: 365.2165 (-6.0 ppm).

$(+)-(R)-2-\{(S)-2-(B e n z y l o x y)-1-[($ tert-butyldimethylsilyl)oxy] ethyl\}-2-methylbut-3-en-1-yl benzoate (15): To a solution of primary alcohol $14\left(3.7 \mathrm{~g}, 10 \mathrm{mmol}, 1.0\right.$ equiv.) in cyclohexane $/ \mathrm{CH}_{2} \mathrm{Cl}_{2}(2: 1,100 \mathrm{~mL}, 0.10 \mathrm{M})$ at $0{ }^{\circ} \mathrm{C}$, benzyl 2,2,2-trichloroacetimidate $(2.4 \mathrm{~mL}, 13 \mathrm{mmol}, 1.3$ equiv.) and trifluoromethanesulfonic acid $(0.13 \mathrm{~mL}$, $1.5 \mathrm{~mL}, 0.15$ equiv.) were added. The resulting suspension was stirred for 16 hours at room temperature at which point $\mathrm{NEt}_{3}(0.7 \mathrm{~mL}, 5 \mathrm{mmol}, 0.5$ equiv.) was added and the volatiles removed in vacuo. Hexane was added, and the solids filtered over Celite. Purification by flash chromatography (Hexanes/Et ${ }_{2} \mathrm{O}$ ) provided 15 as a colorless oil (3.8 g, 84\%): $\mathrm{R}_{f}=0.51$ (Hexanes/EtOAc, 90:10); $[\alpha]_{\mathrm{D}}^{25}+9.8\left(c=3.6, \mathrm{CH}_{2} \mathrm{Cl}_{2}\right.$ ); Molecular formula: $\mathrm{C}_{27} \mathrm{H}_{38} \mathrm{O}_{4} \mathrm{Si}$; MW: 454.68; IR (neat) $v_{\max } 2953,2928,2855,1720,1269,1108 \mathrm{~cm}^{-1} ;{ }^{1} \mathrm{H} \mathrm{NMR}\left(500 \mathrm{MHz}, \mathrm{CDCl}_{3}\right) \delta$ $8.10-8.03(\mathrm{~m}, 2 \mathrm{H}), 7.61-7.56(\mathrm{~m}, 1 \mathrm{H}), 7.51-7.43(\mathrm{~m}, 2 \mathrm{H}), 7.42-7.27(\mathrm{~m}, 5 \mathrm{H}), 6.05(\mathrm{dd}, J 17.7,11.0 \mathrm{~Hz}, 1 \mathrm{H})$, $5.24-5.17(\mathrm{~m}, 2 \mathrm{H}), 4.54(\mathrm{~d}, J 11.9 \mathrm{~Hz}, 1 \mathrm{H}), 4.48(\mathrm{~d}, J 11.9 \mathrm{~Hz}, 1 \mathrm{H}), 4.34(\mathrm{~d}, J 10.6 \mathrm{~Hz}, 1 \mathrm{H}), 4.27(\mathrm{~d}, J 10.6 \mathrm{~Hz}, 1 \mathrm{H})$, 3.99 (dd, J 6.4, $2.9 \mathrm{~Hz}, 1 \mathrm{H}$ ), 3.67 (dd, J 10.0, $3.0 \mathrm{~Hz}, 1 \mathrm{H}$ ), 3.39 (dd, J 10.0, $6.4 \mathrm{~Hz}, 1 \mathrm{H}), 1.22(\mathrm{~s}, 3 \mathrm{H}), 0.92(\mathrm{~s}, 9 \mathrm{H})$, 0.11 (s, 3H), 0.09 (s, 3H) ppm ; ${ }^{13} \mathrm{CNMR}\left(126 \mathrm{MHz}, \mathrm{CDCl}_{3}\right) \delta 166.6,139.5,138.3,133.0,130.6,129.6,128.5$, $128.4,127.8,127.7,115.7,75.9,73.6,73.4,69.2,45.0,26.1,18.8,18.5,-3.8,-5.0$ ppm; HRMS calcd for $\mathrm{C}_{27} \mathrm{H}_{39} \mathrm{O}_{4} \mathrm{Si}\left[\mathrm{M}+\mathrm{H}^{+}\right]: 455.2612$; found 455.2592 (-4.4 ppm).

(+)-(R)-2-\{(S)-2-(Benzyloxy)-1-[(tert-butyldimethylsilyl)oxy]ethyl\}-2-methylbut-3-en-1-ol (S1): To a $-40{ }^{\circ} \mathrm{C}$ solution of benzoate 15 (3.2 g, $7.0 \mathrm{mmol}, 1.0$ equiv.) in $\mathrm{CH}_{2} \mathrm{Cl}_{2}(21 \mathrm{~mL}, 0.33 \mathrm{M})$, DIBAL-H (1.0 M in hexanes, 17 $\mathrm{mL}, 17 \mathrm{mmol}, 2.4$ equiv.) was added over 10 minutes. The solution was stirred for 1 hour at $-40{ }^{\circ} \mathrm{C}$ before addition of $\mathrm{Et}_{2} \mathrm{O}(20 \mathrm{~mL})$ followed by saturated aqueous Rochelle salt $(30 \mathrm{~mL})$. The biphasic mixture was warmed to room temperature and stirred vigorously for 2 hours. The aqueous layer was extracted with $\mathrm{Et}_{2} \mathrm{O}(2$ $\times 25 \mathrm{~mL}$ ) and the combined organic fractions were washed with $0.1 \mathrm{~N} \mathrm{HCl}$, water and brine, dried over $\mathrm{MgSO}_{4}$, filtered and concentrated in vacuo. Purification by flash chromatography provided primary alcohol S1 as a colorless oil (1.5 g, 61\% yield): $\mathrm{R}_{f}=0.41$ (Hexanes/EtOAc, 80:20); $[\alpha]_{\mathrm{D}}^{25}+2.9\left(c=3.0, \mathrm{CH}_{2} \mathrm{Cl}_{2}\right.$ ); Molecular formula: $\mathrm{C}_{20} \mathrm{H}_{34} \mathrm{O}_{3} \mathrm{Si}$; MW: 350.57; IR (neat) $v_{\max } 3455,1471,1096 \mathrm{~cm}^{-1} ;{ }^{1} \mathrm{H}$ NMR $\left(500 \mathrm{MHz}, \mathrm{CDCl}_{3}\right) \delta 7.38-7.28(\mathrm{~m}, 5 \mathrm{H})$, 5.93 (dd, J 17.8, 11.0 Hz, 1H), 5.15 (dd, J 11.1, 1.2 Hz, 1H), 5.08 (dd, J 17.8, 1.3 Hz, 1H), $4.52(d, J 11.9 \mathrm{~Hz}, 1 \mathrm{H}$ ), 4.49 (d, J $11.9 \mathrm{~Hz}, 1 \mathrm{H}), 3.79$ (dd, J 5.4, $3.9 \mathrm{~Hz}, 1 \mathrm{H}), 3.62$ (dd, J 10.1, 3.8 Hz, 2H), $3.57-3.51(\mathrm{~m}, 1 \mathrm{H}), 3.44(\mathrm{dd}, J$ 10.1, $5.5 \mathrm{~Hz}, 1 \mathrm{H}), 2.66(\mathrm{~s}, 1 \mathrm{H}), 1.11(\mathrm{~s}, 3 \mathrm{H}), 0.91(\mathrm{~s}, 9 \mathrm{H}), 0.10(\mathrm{~s}, 3 \mathrm{H}), 0.08(\mathrm{~s}, 3 \mathrm{H}) \mathrm{ppm} ;{ }^{13} \mathrm{C} \mathrm{NMR}(126 \mathrm{MHz}$, $\left.\mathrm{CDCl}_{3}\right) \delta 141.4,138.0,128.5,127.84,127.78,114.8,77.6,73.5,73.0,68.1,45.7,26.1,19.0,18.4,-3.9,-4.9$ ppm; HRMS calcd for $\mathrm{C}_{20} \mathrm{H}_{34} \mathrm{O}_{3} \mathrm{SiNa}\left[\mathrm{M}+\mathrm{Na}^{+}\right]$: 373.2169; found 373.2155 (-3.8 ppm).

(+)-(S)-2-\{(S)-2-(Benzyloxy)-1-[(tert-butyldimethylsilyl)oxy]ethyl\}-2-methylbut-3-enal (6): To a solution of primary alcohol $\mathbf{S 1}\left(0.84 \mathrm{~g}, 2.4 \mathrm{mmol}, 1.0\right.$ equiv.) in $\mathrm{CH}_{2} \mathrm{Cl}_{2}(24 \mathrm{~mL}, 0.10 \mathrm{M})$, Dess-Martin periodinane (DMP, 1.1 $\mathrm{g}, 2.6 \mathrm{mmol}, 1.1$ equiv.) was added. The resulting suspension was stirred for 16 hours at room temperature before addition of saturated aqueous $\mathrm{Na}_{2} \mathrm{~S}_{2} \mathrm{O}_{3}(20 \mathrm{~mL})$. The biphasic mixture was vigorously stirred for 30 minutes. The aqueous layer was extracted with $\mathrm{CH}_{2} \mathrm{Cl}_{2}(1 \times 20 \mathrm{~mL})$, the combined organic fractions were washed with brine, dried over $\mathrm{MgSO}_{4}$, filtered and concentrated in vacuo. The crude aldehyde was purified by flash chromatography (Hexanes/EtOAc) to provide 6 as a colorless oil (700 mg, 84\% yield): $\mathrm{R}_{f}=0.59$ (Hexanes/EtOAc, 88:12); $[\alpha]_{\mathrm{D}}^{25}+2.0\left(c=3.0, \mathrm{CH}_{2} \mathrm{Cl}_{2}\right.$ ); Molecular formula: $\mathrm{C}_{20} \mathrm{H}_{32} \mathrm{O}_{3} \mathrm{Si}$; $\mathrm{MW}$ : 348.56; IR (neat) $v_{\max }$ $1726 \mathrm{~cm}^{-1} ;{ }^{1} \mathrm{H}$ NMR $\left(500 \mathrm{MHz}, \mathrm{CDCl}_{3}\right) \delta 9.58(\mathrm{~s}, 1 \mathrm{H}), 7.37-7.27(\mathrm{~m}, 5 \mathrm{H}), 6.06(\mathrm{dd}, J 17.8,10.9 \mathrm{~Hz}, 1 \mathrm{H}), 5.29(\mathrm{~d}, J$ $10.9 \mathrm{~Hz}, 1 \mathrm{H}), 5.13$ (d, J $17.8 \mathrm{~Hz}, 1 \mathrm{H}), 4.47$ (d, J $11.9 \mathrm{~Hz}, 1 \mathrm{H}), 4.43$ (d, J $11.9 \mathrm{~Hz}, 1 \mathrm{H}$ ), 4.10 (t, J $5.2 \mathrm{~Hz}, 1 \mathrm{H}), 3.46$ (dd, J 9.9, $5.1 \mathrm{~Hz}, 1 \mathrm{H}), 3.42(\mathrm{dd}, J$ 9.9, $5.4 \mathrm{~Hz}, 1 \mathrm{H}), 1.18(\mathrm{~s}, 3 \mathrm{H}), 0.86(\mathrm{~s}, 9 \mathrm{H}), 0.06(\mathrm{~s}, 3 \mathrm{H}), 0.04(\mathrm{~s}, 3 \mathrm{H}) \mathrm{ppm} ;{ }^{13} \mathrm{C}$ NMR $\left(126 \mathrm{MHz} \mathrm{CDCl}_{3}\right) \delta 202.0,137.9,137.0,128.5,127.83,127.80,117.3,75.5,73.5,72.1,56.9,26.0,18.3$, 14.6, -4.0, -4.9 ppm; HRMS calcd for $\mathrm{C}_{20} \mathrm{H}_{32} \mathrm{O}_{3} \mathrm{SiNa}\left[\mathrm{M}+\mathrm{Na}^{+}\right]$: 371.2013 ; found 371.2000 (-3.5 ppm). 


\section{Compounds 5a and 16 from Scheme 5:}

\section{$(-)-(2 R, 3 R)-3-\{(S)-2-(B e n z y l o x y)-1-[(t e r t-b u t y l d i m e t h y l s i l y l) o x y]$ ethyl\}-2-hydroxy-3-methylpent-4-enenitrile} (5a), and (2S,3R)-1-(benzyloxy)-2-[(tert-butyldimethylsilyl)oxy]-3-[(S)-cyano(hydroxy)methyl]-3-methylpent4-en-1-ylium (5b): A solution of aldehyde 6 (700 mg, $2.01 \mathrm{mmol}, 1.00$ equiv.) in $\mathrm{CH}_{2} \mathrm{Cl}_{2}(20 \mathrm{~mL}, 0.10 \mathrm{M})$ was cooled to $-15^{\circ} \mathrm{C}\left(\mathrm{NH}_{4} \mathrm{Cl} /\right.$ ice $)$ before addition of $\mathrm{MgBr}_{2} \cdot \mathrm{OEt}_{2}(2.6 \mathrm{~g}, 10 \mathrm{mmol}, 5.0$ equiv.) in one portion. After 10 minutes, TMSCN $(0.50 \mathrm{~mL}, 4.0 \mathrm{mmol}, 2.0$ equiv.) was added dropwise and the resulting mixture was stirred 1 hour at $-15{ }^{\circ} \mathrm{C}$. The reaction was quenched with saturated aqueous $\mathrm{NaHCO}_{3}(20 \mathrm{~mL})$ and the biphasic mixture warmed to room temperature. After stirring vigorously for 15 minutes, $\mathrm{Et}_{2} \mathrm{O}(20 \mathrm{~mL})$ was added and the aqueous layer was extracted with $\mathrm{Et}_{2} \mathrm{O}(1 \times 15 \mathrm{~mL})$. The combined organic fractions were washed with brine, dried over $\mathrm{MgSO}_{4}$, filtered and concentrated in vacuo to afford the crude cyanohydrin which could be used directly for the next step. ${ }^{1} \mathrm{H}$ NMR analysis of the crude mixture showed an 8:1 mixture of stereoisomers. Data for the major 2,4-syn-cyanohydrin $5 \mathrm{a}\left([\alpha]_{\mathrm{D}}^{25}-1.9\left(c=5.5, \mathrm{CH}_{2} \mathrm{Cl}_{2}\right)\right)$ correspond to that obtained from the reaction of racemic aldehyde 6 with $\mathrm{MgBr}_{2} \cdot \mathrm{OEt}_{2}$ at $0^{\circ} \mathrm{C}$ (Table 1 , entry 1 ) as seen below.

(+)-(2R,3R)-3-((S)-2-(Benzyloxy)-1-((tert-butyldimethylsilyl)oxy)ethyl)-2-((tert-butyldimethylsilyl)oxy)-3-methylpent-4-enenitrile (16): The crude cyanohydrin 5 (755 mg, $2.01 \mathrm{mmol}, 1.00$ equiv.) was dissolved in $\mathrm{CH}_{2} \mathrm{Cl}_{2}(10$ $\mathrm{mL}, 0.20 \mathrm{M})$ and the resulting solution was cooled to $0{ }^{\circ} \mathrm{C}$ followed by addition of pyridine $(0.49 \mathrm{~mL}, 6.0 \mathrm{mmol}$, 3.0 equiv.) and TBSOTf ( $0.55 \mathrm{~mL}, 2.4 \mathrm{mmol}, 1.2$ equiv.). The reaction was stirred at $0{ }^{\circ} \mathrm{C}$ for 16 hours followed by addition of saturated aqueous $\mathrm{NaHCO}_{3}(15 \mathrm{~mL})$ and hexanes $(15 \mathrm{~mL})$. The aqueous layer was extracted with Hexanes/Et $2 \mathrm{O}(2: 1,2 \times 15 \mathrm{~mL})$ and the combined organic fractions were washed with $0.1 \mathrm{~N} \mathrm{HCl}$ followed by brine, dried over $\mathrm{MgSO}_{4}$, filtered and concentrated in vacuo. Purification by flash chromatography (Hexanes/Et ${ }_{2} \mathrm{O}$ ) provided the protected cyanohydrin 16 as a single diastereoisomer $(815 \mathrm{mg}, 83 \%$ yield over two steps): $\mathrm{R}_{f}=0.52$ (Hexanes/Et $\left.{ }_{2} \mathrm{O}, 95: 5\right) ;[\alpha]_{\mathrm{D}}^{25}+36$ (c= 2.3, $\mathrm{CH}_{2} \mathrm{Cl}_{2}$ ); Molecular formula: $\mathrm{C}_{27} \mathrm{H}_{47} \mathrm{NO}_{3} \mathrm{Si}_{2} ; \mathrm{MW}$ 489.85; IR (neat) $v_{\max } 2953,2929,2857,1253,1118,1102 \mathrm{~cm}^{-1} ;{ }^{1} \mathrm{H} \mathrm{NMR}\left(500 \mathrm{MHz}, \mathrm{CDCl}_{3}\right) \delta 7.36-7.26$ (m, $5 \mathrm{H}), 5.89$ (dd, J 17.6, 10.9 Hz, 1H), 5.24 (dd, J 10.9, 1.0 Hz, 1H), 5.13 (dd, J 17.6, 1.2 Hz, 1H), $4.71(\mathrm{~s}, 1 \mathrm{H}), 4.48$ (d, J $11.9 \mathrm{~Hz}, 1 \mathrm{H}), 4.41$ (d, J $11.9 \mathrm{~Hz}, 1 \mathrm{H}), 3.99$ (dd, J 6.7, $2.5 \mathrm{~Hz}, 1 \mathrm{H}), 3.51$ (dd, J 10.2, $2.6 \mathrm{~Hz}, 1 \mathrm{H}), 3.27$ (dd, J 10.2, 6.7 Hz, 1H), $1.16(\mathrm{~s}, 3 \mathrm{H}), 0.90(\mathrm{~s}, 9 \mathrm{H}), 0.88(\mathrm{~s}, 9 \mathrm{H}), 0.16(\mathrm{~s}, 6 \mathrm{H}), 0.08(\mathrm{~s}, 3 \mathrm{H}), 0.06(\mathrm{~s}, 3 \mathrm{H}) \mathrm{ppm} ;{ }^{13} \mathrm{C} \mathrm{NMR}$ $\left(126 \mathrm{MHz}, \mathrm{CDCl}_{3}\right) \delta 138.1,137.3,128.5,127.7,119.2,117.9,76.5,73.4,73.2,67.0,49.5,26.2,25.7,18.5,18.2$, 14.9, -3.8, -5.10, -5.11, -5.3 ppm; HRMS calcd for $\mathrm{C}_{27} \mathrm{H}_{47} \mathrm{NO}_{3} \mathrm{Si}_{2} \mathrm{Na}\left[\mathrm{M}+\mathrm{Na}^{+}\right]$: 512.2987; found 512.2963 (-4.6 ppm).

Aldehydes 17, 19, 21, 23, 25 and 27 along with cyanohydrins 5a,b, 18a,b, 20a,b, 22a,b, 24a,b, 26a,b and 28a,b from Table 1 (racemic):

(士)-(S)-Ethyl 2-((S)-2-(Benzyloxy)-1-(tert-butyldimethylsilyloxy)ethyl)-2-methylbut-3-enoate (S2): To a solution of (S)-methyl 2-[(S)-2-(benzyloxy)-1-hydroxyethyl]-2-methylbut-3-enoate ${ }^{22}$ (4.29 g, $16.2 \mathrm{mmol}, 1.00$ equiv.) in anhydrous $\mathrm{CH}_{2} \mathrm{Cl}_{2}(160 \mathrm{~mL}, 0.10 \mathrm{M})$ at $0{ }^{\circ} \mathrm{C}, 2,6$-lutidine $(3.8 \mathrm{~mL}, 33 \mathrm{mmol}, 2.0$ equiv.) and TBSOTf $(5.22 \mathrm{~mL}, 22.7 \mathrm{mmol}, 1.40$ equiv.) were added. The solution was stirred until the alcohol was all consumed, $1 \mathrm{~h} 30$ at $0{ }^{\circ} \mathrm{C}$, as determined by TLC. An aqueous solution of $\mathrm{NH}_{4} \mathrm{Cl}$ was added to the reaction mixture and the aqueous layer was extracted with $\mathrm{Et}_{2} \mathrm{O}(3 \mathrm{x})$. The organic layers were combined, dried over $\mathrm{MgSO}_{4}$, filtered and concentrated in vacuo. Purification by flash chromatography using 5:95 EtOAc:Hex provided protected ester S2 as a clear oil (5.1 g, 83\% yield): $\mathrm{R}_{f}=0.3$ (5:95 EtOAc:Hex); Molecular formula: $\mathrm{C}_{21} \mathrm{H}_{34} \mathrm{O}_{4} \mathrm{Si}$; MW: 378.58; IR (neat) $v_{\max } 1737 \mathrm{~cm}^{-1} ;{ }^{1} \mathrm{H}$ NMR $\left(500 \mathrm{MHz}, \mathrm{CDCl}_{3}\right) \delta 7.37-7.27(\mathrm{~m}, 5 \mathrm{H}), 6.10$ (dd, J 17.6, $\left.10.8 \mathrm{~Hz}, 1 \mathrm{H}\right), 5.17$ (dd, J $10.8,0.9 \mathrm{~Hz}, 1 \mathrm{H}), 5.08(\mathrm{dd}, J 17.6,1.0 \mathrm{~Hz}, 1 \mathrm{H}), 4.47(\mathrm{~d}, J 11.8 \mathrm{~Hz}, 1 \mathrm{H}), 4.41(\mathrm{~d}, J 11.8 \mathrm{~Hz}, 1 \mathrm{H}), 4.24(\mathrm{dd}, J 6.2,4.8$ $\mathrm{Hz}, 1 \mathrm{H}), 3.59$ (s, 3H), 3.44 (dd, J 10.0, $4.8 \mathrm{~Hz}, 1 \mathrm{H}), 3.35$ (dd, J 10.0, 6.2 Hz, 1H), $1.26(\mathrm{~s}, 3 \mathrm{H}), 0.84(\mathrm{~s}, 9 \mathrm{H}), 0.04(\mathrm{~s}$, $6 \mathrm{H}) ;{ }^{13} \mathrm{C}$ NMR $\left(126 \mathrm{MHz} \mathrm{CDCl}_{3}\right) \delta 174.9,139.5,138.2,128.4,127.8,127.7,115.0,75.7,73.4,72.8,53.5,52.0$, 26.0, 18.3, 15.8, -3.9, -4.9 ppm; HRMS calcd for $\mathrm{C}_{21} \mathrm{H}_{35} \mathrm{O}_{4} \mathrm{Si}\left[\mathrm{M}+\mathrm{H}^{+}\right]$: 379.2299 , found 379.2301 (0.6 ppm). 
(士)-(R)-2-[(S)-2-(Benzyloxy)-1-(tert-butyldimethylsilyloxy)ethyl]-2-methylbut-3-en-1-ol $\quad$ (S1): Following General Procedure A and purification by flash chromatography using 10:90 EtOAc:Hex, racemic alcohol S1 (4.5 g, 95\% yield) was obtained as a clear oil. ${ }^{1} \mathrm{H}$ NMR and ${ }^{13} \mathrm{C}$ NMR as reported above for enantiopure compound. (士)-(S)-2-[(S)-2-(Benzyloxy)-1-(tert-butyldimethylsilyloxy)ethyl]-2-methylbut-3-enal (6): Following General Procedure B and purification by flash chromatography using 20:80 EtOAc:Hex, racemic aldehyde 6 (4.4 g, $97 \%$ yield) was obtained as a clear oil. ${ }^{1} \mathrm{H} N M R$ and ${ }^{13} \mathrm{C}$ NMR as reported above for enantiopure compound.

(士)-(2R,3R)-3-[(S)-2-(Benzyloxy)-1-(tert-butyldimethylsilyloxy)ethyl]-2-hydroxy-3-methylpent-4-enenitrile (5a) and ( \pm )-(2S,3R)-3-[(S)-2-(benzyloxy)-1-(tert-butyldimethylsilyloxy)ethyl]-2-hydroxy-3-methylpent-4-enenitrile (5b): Cyanohydrins $\mathbf{5 a}$ and $\mathbf{5 b}$ were prepared following General Procedure C. ${ }^{1} \mathrm{H}$ NMR spectroscopic analysis of the crude reaction indicated a 6:1 mixture of 2,4-syn and anti diastereomers (Table 1, entry 1). Purification by flash chromatography using 10:90 EtOAc:Hex provided 5a and $\mathbf{5 b}$ as a clear oil $(69.1 \mathrm{mg}, 79 \%$ yield). 5a: $\mathrm{R}_{f}=0.15$ (10:90 EtOAc:Hex); Molecular formula: $\mathrm{C}_{21} \mathrm{H}_{33} \mathrm{NO}_{3} \mathrm{Si}$; MW: 375.58; IR (neat) $v_{\max } 3447$, $2362 \mathrm{~cm}^{-1} ;{ }^{1} \mathrm{H}$ NMR $\left(500 \mathrm{MHz}, \mathrm{CDCl}_{3}\right) \delta 7.40-7.29(\mathrm{~m}, 5 \mathrm{H}), 6.02(\mathrm{dd}, J 17.6,11.0 \mathrm{~Hz}, 1 \mathrm{H}), 5.34(\mathrm{dd}, J 11.0,0.9$ $\mathrm{Hz}, 1 \mathrm{H}), 5.27(\mathrm{dd}, J 17.6,0.9 \mathrm{~Hz}, 1 \mathrm{H}), 4.58(\mathrm{~d}, J 4.8 \mathrm{~Hz}, 1 \mathrm{H}), 4.55(\mathrm{~d}, J 11.7 \mathrm{~Hz}, 1 \mathrm{H}), 4.51(\mathrm{~d}, J 11.7 \mathrm{~Hz}, 1 \mathrm{H}), 4.15$ (d, J $5.3 \mathrm{~Hz}, 1 \mathrm{H}$ ), 3.87 (dd, J 5.3, 3.9 Hz, 1H), 3.55 (dd, J 10.4, 5.2 Hz, 1H), 3.47 (dd, J 10.4, 3.9 Hz, $1 \mathrm{H}$ ), 1.20 (s, $3 \mathrm{H}), 0.88(\mathrm{~s}, 9 \mathrm{H}), 0.09$ (s, 3H), $0.03(\mathrm{~s}, 3 \mathrm{H}) \mathrm{ppm} ;{ }^{13} \mathrm{C} \mathrm{NMR}\left(126 \mathrm{MHz}, \mathrm{CDCl}_{3}\right) \delta 137.7,136.8,128.7,128.3,128.1$, 118.8, 118.2, 76.1, 73.8, 71.4, 66.7, 49.1, 26.0, 18.3, 17.0, -4.1, -4.9 ppm; HRMS calcd for $\mathrm{C}_{21} \mathrm{H}_{34} \mathrm{O}_{3} \mathrm{NSi}\left[\mathrm{M}+\mathrm{H}^{+}\right]$: 376.2302, found 376.2303 (0.04 ppm). 5b: $R_{f}=0.19$ (10:90 EtOAc:Hex); IR (neat) $v_{\max } 3424,2247 \mathrm{~cm}^{-1} ;{ }^{1} \mathrm{H} \mathrm{NMR}$ $\left(500 \mathrm{MHz}, \mathrm{CDCl}_{3}\right) \delta 7.39-7.27(\mathrm{~m}, 5 \mathrm{H}), 5.89(\mathrm{dd}, J 17.6,11.0 \mathrm{~Hz}, 1 \mathrm{H}), 5.32(\mathrm{~d}, J 11.0 \mathrm{~Hz}, 1 \mathrm{H}), 5.27(\mathrm{~d}, J 17.6 \mathrm{~Hz}$, $1 \mathrm{H}), 4.70(\mathrm{~d}, J 5.7 \mathrm{~Hz}, 1 \mathrm{H}), 4.48(\mathrm{~s}, 2 \mathrm{H}), 3.95(\mathrm{dd}, J 4.9,3.9 \mathrm{~Hz}, 1 \mathrm{H}), 3.81(\mathrm{~d}, J 6.5 \mathrm{~Hz}, 1 \mathrm{H}), 3.55(\mathrm{dd}, J 10.3,3.9 \mathrm{~Hz}$, $1 \mathrm{H}), 3.43$ (dd, J 10.4, $5.0 \mathrm{~Hz}, 1 \mathrm{H}), 1.27(\mathrm{~s}, 3 \mathrm{H}), 0.89(\mathrm{~s}, 9 \mathrm{H}), 0.13(\mathrm{~s}, 3 \mathrm{H}), 0.08(\mathrm{~s}, 3 \mathrm{H}) ;{ }^{13} \mathrm{C} \mathrm{NMR}\left(126 \mathrm{MHz} \mathrm{CDCl}_{3}\right)$ $\delta$ 137.6, 137.1, 128.6, 128.0, 127.9, 118.9, 118.2, 77.4, 73.5, 72.3, 68.0, 47.6, 26.0, 18.3, 16.5, -4.1, -4.9 ppm; HRMS calcd for $\mathrm{C}_{21} \mathrm{H}_{34} \mathrm{O}_{3} \mathrm{NSi}\left[\mathrm{M}+\mathrm{H}^{+}\right]$: 376.2302 , found 376.2302 (-0.2 ppm).

(士)-(S)-Methyl 2-[(S)-1,2-bis(benzyloxy)ethyl]-2-methylbut-3-enoate (S3): To a solution of (S)-methyl 2-[(S)-2(benzyloxy)-1-hydroxyethyl]-2-methylbut-3-enoate ${ }^{22}(0.32 \mathrm{~g}, 1.2 \mathrm{mmol}, 1.0$ equiv.) in a mixture of $2: 1$ cyclohexane:DCM $\left(6.1 \mathrm{~mL}, 0.20 \mathrm{M}\right.$ ) at $0^{\circ} \mathrm{C}$, benzyl-2,2,2-trichloroacetimidate (340 $\mu \mathrm{L}, 1.80 \mathrm{mmol}, 1.80$ equiv.) and triflic acid (11 $\mu \mathrm{L}, 0.12 \mathrm{mmol}, 0.10$ equiv.) were added. The solution was stirred overnight at $0{ }^{\circ} \mathrm{C}$. Triethylamine ( $100 \mu \mathrm{L}, 0.61 \mathrm{mmol}, 0.50$ equiv.) was added and the reaction mixture was then concentrated. Purification by flash chromatography using 15:85 (EtOAc:Hex): provided protected ester S3 as a clear oil (0.36 g, 84\% yield): $\mathrm{R}_{f}=0.28$ (15:85 EtOAc:Hex); Molecular formula: $\mathrm{C}_{22} \mathrm{H}_{26} \mathrm{O}_{4}$; MW: 354.45; IR (neat) $v_{\max } 3030$, 2949, 2865, 1734, $1117 \mathrm{~cm}^{-1}$; ${ }^{1} \mathrm{H}$ NMR $\left(500 \mathrm{MHz}, \mathrm{CDCl}_{3}\right) \delta 7.48-7.06(\mathrm{~m}, 10 \mathrm{H}), 6.15(\mathrm{dd}, J 17.6,10.9 \mathrm{~Hz}, 1 \mathrm{H})$, $5.16(d, J 10.8 \mathrm{~Hz}, 1 \mathrm{H}), 5.09$ (d, J $17.6 \mathrm{~Hz}, 1 \mathrm{H}), 4.76$ (d, J $11.5 \mathrm{~Hz}, 1 \mathrm{H}), 4.57$ (d, J $11.5 \mathrm{~Hz}, 1 \mathrm{H}), 4.47$ (d, J $11.9 \mathrm{~Hz}$, $1 \mathrm{H}), 4.43(\mathrm{~d}, J 11.9 \mathrm{~Hz}, 1 \mathrm{H}), 4.03(\mathrm{dd}, J 6.0,4.6 \mathrm{~Hz}, 1 \mathrm{H}), 3.57(\mathrm{~s}, 3 \mathrm{H}), 3.54(\mathrm{~d}, J=6.1 \mathrm{~Hz}, 1 \mathrm{H}), 3.53(\mathrm{~d}, J 4.5 \mathrm{~Hz}$, $1 \mathrm{H}), 1.28(\mathrm{~s}, 3 \mathrm{H}) \mathrm{ppm} ;{ }^{13} \mathrm{C} \mathrm{NMR}\left(126 \mathrm{MHz}, \mathrm{CDCl}_{3}\right) \delta 174.9,138.9,138.6,138.3,128.5,128.3,127.72,127.71$, $127.65,127.5,115.2,82.8,74.2,73.5,71.8,52.9,52.2,16.8$ ppm; HRMS calcd for $\mathrm{C}_{22} \mathrm{H}_{27} \mathrm{O}_{4}\left[\mathrm{M}+\mathrm{H}^{+}\right]: 355.1904$, found: 355.1912 (2.4 ppm).

$( \pm)-(R)-2-[(S)-1,2-B i s(b e n z y l o x y)$ ethyl]-2-methylbut-3-en-1-ol (S4): Following General Procedure $A$ and purification by flash chromatography using 25:75 EtOAc:Hex, provided primary alcohol S4 as a clear oil (58.3 mg, 98\% yield): $R_{f}=0.23$ (20:80 EtOAc:Hex); Molecular formula: $\mathrm{C}_{21} \mathrm{H}_{26} \mathrm{O}_{3} ; \mathrm{MW}: 326.44$; IR (neat) Umax 3443, 2871, $1091 \mathrm{~cm}^{-1}$; ${ }^{1} \mathrm{H}$ NMR $\left(500 \mathrm{MHz}, \mathrm{CDCl}_{3}\right) \delta 7.39-7.26(\mathrm{~m}, 10 \mathrm{H}), 5.98(\mathrm{dd}, J 17.7,11.0 \mathrm{~Hz}, 1 \mathrm{H}), 5.15(\mathrm{dd}, J$ 11.0, $1.3 \mathrm{~Hz}, 1 \mathrm{H}), 5.09(\mathrm{dd}, J 17.8,1.2 \mathrm{~Hz}, 1 \mathrm{H}), 4.83(\mathrm{~d}, J 11.5 \mathrm{~Hz}, 1 \mathrm{H}), 4.58(\mathrm{~d}, J 11.5 \mathrm{~Hz}, 1 \mathrm{H}), 4.53(\mathrm{~s}, 2 \mathrm{H}), 3.71$ (app.q, J $5.9 \mathrm{~Hz}, 1 \mathrm{H}$ ), 3.61 (app.q, J $5.9 \mathrm{~Hz}, 2 \mathrm{H}$ ), $3.59-3.55(\mathrm{~m}, 1 \mathrm{H}), 3.51$ (dd, J 10.9, $5.5 \mathrm{~Hz}, 1 \mathrm{H}), 2.46-2.41$ (m, $1 \mathrm{H}), 1.08$ (s, 3H) ppm; ${ }^{13} \mathrm{C} \mathrm{NMR}\left(126 \mathrm{MHz} \mathrm{CDCl}_{3}\right) \delta 140.6,138.6,138.2,128.57,128.53,128.0,127.84,127.82$, 
127.76, 115.0, 83.7, 73.6, 73.6, 71.8, 68.8, 45.5, 18.8 ppm; HRMS calcd for $\mathrm{C}_{21} \mathrm{H}_{26} \mathrm{O}_{3} \mathrm{Na}\left[\mathrm{M}+\mathrm{Na}^{+}\right]: 349.1774$, found: 349.1781 (1.9 ppm).

(士)-(S)-2-[(S)-1,2-Bis(benzyloxy)ethyl]-2-methylbut-3-enal (17): Following General Procedure $B$ and purification by flash chromatography using 20:80 EtOAc:Hex, provided aldehyde 17 as a clear oil (1.82 g, 98\% yield): $\mathrm{R}_{f}=0.41$ (30:70 EtOAc:Hex); Molecular formula: $\mathrm{C}_{21} \mathrm{H}_{24} \mathrm{O}_{3} ; \mathrm{MW}: 324.42 ; \mathrm{R}$ (neat) $\mathrm{v}_{\max } 2865,1725,1095$ $\mathrm{cm}^{-1} ;{ }^{1} \mathrm{H}$ NMR $\left(500 \mathrm{MHz}, \mathrm{CDCl}_{3}\right) \delta 9.54(\mathrm{~s}, 1 \mathrm{H}), 7.38-7.27(\mathrm{~m}, 10 \mathrm{H}), 6.10(\mathrm{dd}, J 17.8,10.9 \mathrm{~Hz}, 1 \mathrm{H}), 5.32(\mathrm{dd}, J$ 10.9, $0.8 \mathrm{~Hz}, 1 \mathrm{H}), 5.16(\mathrm{dd}, J 17.8,0.8 \mathrm{~Hz}, 1 \mathrm{H}), 4.77(\mathrm{~d}, J 11.5 \mathrm{~Hz}, 1 \mathrm{H}), 4.58(\mathrm{~d}, J 11.5 \mathrm{~Hz}, 1 \mathrm{H}), 4.49(\mathrm{~s}, 2 \mathrm{H}), 3.88$ (t, J $4.9 \mathrm{~Hz}, 1 \mathrm{H}), 3.61$ (app.s, 1H), 3.60 (app.s, $1 \mathrm{H}), 1.20$ (s, 3H); ${ }^{13} \mathrm{C} N M R\left(126 \mathrm{MHz}^{\mathrm{CDCl}}{ }_{3}\right) \delta 201.8,138.4$, 138.0, 136.8, 128.6, 128.4, 127.90, 127.86, 127.8 (one aromatic carbon missing), 117.7, 81.8, 73.7, 73.6, 70.4, 56.3, 15.4 ppm; HRMS calcd for $\mathrm{C}_{21} \mathrm{H}_{24} \mathrm{O}_{3} \mathrm{Na}\left[\mathrm{M}+\mathrm{Na}^{+}\right]$: 347.1618 , found: 347.1619 (0.4 ppm).

$( \pm)-(2 R, 3 R)-3-[(S)-1,2-B i s($ benzyloxy)ethyl]-2-hydroxy-3-methylpent-4-enenitrile (18a) and ( \pm$)-(2 S, 3 R)-3-[(S)-$ 1,2-bis(benzyloxy)ethyl]-2-hydroxy-3-methylpent-4-enenitrile (18b): Cyanohydrins 18a and 18b were prepared following General Procedure C. ${ }^{1} \mathrm{H}$ NMR spectroscopic analysis of the crude reaction indicated an 8:1 mixture of 2,4-syn and anti diastereomers (Table 1, entry 3). Purification by flash chromatography using 20:80 EtOAc:Hex, provided 18a and 18b (44.2 mg, 92\% yield) as a clear oil. 18a: $\mathrm{R}_{f}=0.22$ (20:80 EtOAc:Hex); Molecular formula: $\mathrm{C}_{22} \mathrm{H}_{25} \mathrm{NO}_{3} ; \mathrm{MW}: 351.45$; IR (neat) $v_{\max } 3419,2244 \mathrm{~cm}^{-1} ;{ }^{1} \mathrm{H}_{\mathrm{NMR}}\left(500 \mathrm{MHz}, \mathrm{CDCl}_{3}\right) \delta 7.39-$ $7.28(\mathrm{~m}, 10 \mathrm{H}), 6.04(\mathrm{dd}, J 17.6,11.0 \mathrm{~Hz}, 1 \mathrm{H}), 5.36(\mathrm{~d}, J 11.0 \mathrm{~Hz}, 1 \mathrm{H}), 5.28(\mathrm{~d}, J 17.6 \mathrm{~Hz}, 1 \mathrm{H}), 4.76(\mathrm{~d}, J 11.3 \mathrm{~Hz}$, $1 \mathrm{H}), 4.62(\mathrm{~d}, J 4.9 \mathrm{~Hz}, 1 \mathrm{H}), 4.56(\mathrm{~d}, J 11.9 \mathrm{~Hz}, 1 \mathrm{H}), 4.53(\mathrm{~d}, J 11.3 \mathrm{~Hz}, 1 \mathrm{H}), 4.52(\mathrm{~d}, J 11.8 \mathrm{~Hz}, 1 \mathrm{H}), 3.78(\mathrm{~d}, J 5.0 \mathrm{~Hz}$, $1 \mathrm{H}), 3.72-3.65(\mathrm{~m}, 2 \mathrm{H}), 3.60(\mathrm{dd}, J 10.2,4.0 \mathrm{~Hz}, 1 \mathrm{H}), 1.23(\mathrm{~s}, 3 \mathrm{H}) \mathrm{ppm} ;{ }^{13} \mathrm{C} \mathrm{NMR}\left(126 \mathrm{MHz}, \mathrm{CDCl}_{3}\right) \delta 137.8$, 137.2, 136.9, 128.7, 128.6, 128.3, 128.13, 128.12, 128.0, 118.57, 118.53, 82.6, 73.8, 73.5, 69.8, 67.2, 48.5, 17.1 ppm; HRMS calcd for $\mathrm{C}_{22} \mathrm{H}_{25} \mathrm{O}_{3} \mathrm{NNa}\left[\mathrm{M}+\mathrm{Na}^{+}\right]$: 374.1727, found 374.1730 (0.8 ppm). 18b: $\mathrm{R}_{f}=0.18$ (20:80 EtOAc:Hex); IR (neat) $v_{\max } 3420,2244 \mathrm{~cm}^{-1} ;{ }^{1} \mathrm{H} N M R\left(500 \mathrm{MHz}, \mathrm{CDCl}_{3}\right) \delta 7.41-7.28(\mathrm{~m}, 10 \mathrm{H}), 6.02$ (dd, J 17.6, $11.0 \mathrm{~Hz}, 1 \mathrm{H}), 5.31(\mathrm{~d}, J 11.0 \mathrm{~Hz}, 1 \mathrm{H}), 5.24(\mathrm{~d}, J 17.7 \mathrm{~Hz}, 1 \mathrm{H}), 4.89(\mathrm{~d}, J 11.1 \mathrm{~Hz}, 1 \mathrm{H}), 4.65(\mathrm{~d}, J 11.1 \mathrm{~Hz}, 1 \mathrm{H}), 4.53$ $(\mathrm{s}, 2 \mathrm{H}), 4.40(\mathrm{~d}, J 8.8 \mathrm{~Hz}, 1 \mathrm{H}), 3.96(\mathrm{dd}, J 5.6,3.3 \mathrm{~Hz}, 1 \mathrm{H}), 3.80(\mathrm{~d}, J 8.8 \mathrm{~Hz}, 1 \mathrm{H}), 3.63(\mathrm{dd}, J 10.8,3.3 \mathrm{~Hz}, 1 \mathrm{H}), 3.59$ (dd, J 10.8, 5.6 Hz, 1H), 1.22 (s, 3H) ppm; ${ }^{13} \mathrm{C} \mathrm{NMR} \mathrm{(126} \mathrm{MHz,} \mathrm{CDCl} 3$ ) $\delta 137.8,137.6,136.0,128.9,128.7,128.5$, 128.4, 128.0, 127.8, 118.9, 118.0, 83.1, 73.9, 73.6, 71.6, 69.4, 47.0, 17.2 ppm; HRMS calcd for $\mathrm{C}_{22} \mathrm{H}_{25} \mathrm{O}_{3} \mathrm{NNa}$ $\left[\mathrm{M}+\mathrm{Na}^{+}\right]$: 374.1727 , found 374.1730 (0.9 ppm).

(士)-(S)-Methyl 2 [(S)-2-(benzyloxy)-1-(4-methoxybenzyloxy)ethyl]-2-methylbut-3-enoate (S5): To a solution of $(S)$-methyl 2-[(S)-2-(benzyloxy)-1-hydroxyethyl]-2-methylbut-3-enoate 22 (2.0 g, 7.7 mmol, 1.0 equiv.) in dry ether $(77 \mathrm{~mL}, 0.10 \mathrm{M})$ at $0{ }^{\circ} \mathrm{C}, 4$-methoxybenzyl-2,2,2-trichloroacetimidate (6.5 g, $23 \mathrm{mmol}, 3.0$ equiv.) and triflic acid ( $3.4 \mu \mathrm{L}, 0.039 \mathrm{mmol}, 0.0050$ equiv.) were added. The solution was stirred until the alcohol was all consumed, $1 \mathrm{~h} 30$ at $0{ }^{\circ} \mathrm{C}$, as determined by TLC. Triethylamine ( $11 \mu \mathrm{L}, 0.080 \mathrm{mmol}, 0.010$ equiv.) was added to the reaction at $0{ }^{\circ} \mathrm{C}$. An aqueous solution of $\mathrm{NaHCO}_{3}$ was added and the aqueous layer was extracted with $\mathrm{Et}_{2} \mathrm{O}(3 \mathrm{x})$. The organic layers were combined, dried over $\mathrm{MgSO}_{4}$, filtered and concentrated in vacuo. Purification by flash chromatography using 20:80 EtOAc:Hex provided protected ester S5 as a clear oil (2.09 g, 70\% yield): $\mathrm{R}_{f}=0.25$ (20:80 EtOAc:Hex); Molecular formula: $\mathrm{C}_{23} \mathrm{H}_{28} \mathrm{O}_{5} ; \mathrm{MW}: 384.47 ;$ IR (neat) $v_{\max } 1734 \mathrm{~cm}^{-1}$; ${ }^{1} \mathrm{H}$ NMR $\left(500 \mathrm{MHz}, \mathrm{CDCl}_{3}\right) \delta 7.39-7.27(\mathrm{~m}, 5 \mathrm{H}), 7.23(\mathrm{~d}, J 8.5 \mathrm{~Hz}, 2 \mathrm{H}), 6.86(\mathrm{~d}, J 8.6 \mathrm{~Hz}, 2 \mathrm{H}), 6.17$ (dd, J 17.6, $10.8 \mathrm{~Hz}, 1 \mathrm{H}), 5.20(\mathrm{~d}, J 10.8 \mathrm{~Hz}, 1 \mathrm{H}), 5.13(\mathrm{~d}, J 17.6 \mathrm{~Hz}, 1 \mathrm{H}), 4.72(\mathrm{~d}, J 11.0 \mathrm{~Hz}, 1 \mathrm{H}), 4.55(\mathrm{~d}, J 11.0 \mathrm{~Hz}, 1 \mathrm{H}), 4.52$ (d, J $11.9 \mathrm{~Hz}, 1 \mathrm{H}), 4.48$ (d, J $11.6 \mathrm{~Hz}, 1 \mathrm{H}), 4.05$ (dd, J 5.9, $4.7 \mathrm{~Hz}, 1 \mathrm{H}), 3.80(\mathrm{~s}, 3 \mathrm{H}), 3.62(\mathrm{~s}, 3 \mathrm{H}), 3.60-3.53(\mathrm{~m}$, 2H), $1.32(\mathrm{~s}, 3 \mathrm{H}) ;{ }^{13} \mathrm{C}$ NMR $\left(126 \mathrm{MHz}, \mathrm{CDCl}_{3}\right) \delta 174.9,159.2,138.7,138.3,131.0,129.3,128.5,127.7,115.2$, 113.7, 82.4, 73.9, 73.5, 71.8, 55.38, 55.36, 52.9, 52.1, 16.7 ppm; HRMS calcd for $\mathrm{C}_{23} \mathrm{H}_{28} \mathrm{O}_{5} \mathrm{Na}\left[\mathrm{M}+\mathrm{Na}^{+}\right]$: 407.1829, found 407.1837 (1.9 ppm).

(士)-(R)-2-[(S)-2-(Benzyloxy)-1-(4-methoxybenzyloxy)ethyl]-2-methylbut-3-en-1-ol (S6): Following General Procedure A and purification by flash chromatography using 20:80 EtOAc:Hex, provided primary alcohol S6 as 
a clear oil (1.1 g, quantitative yield): $\mathrm{R}_{f}=0.2$ (30:70 EtOAc:Hex); Molecular formula: $\mathrm{C}_{22} \mathrm{H}_{28} \mathrm{O}_{4} ; \mathrm{MW}: 356.46 ;$ IR (neat) $v_{\max } 3451 \mathrm{~cm}^{-1} ;{ }^{1} \mathrm{H} \mathrm{NMR}\left(500 \mathrm{MHz}, \mathrm{CDCl}_{3}\right) \delta 7.41-7.28(\mathrm{~m}, 5 \mathrm{H}), 7.27(\mathrm{~d}, J 8.7 \mathrm{~Hz}, 2 \mathrm{H}), 6.88(\mathrm{~d}, J 8.6 \mathrm{~Hz}$, 2H), $5.97(\mathrm{dd}, J 17.7,11.0 \mathrm{~Hz}, 1 \mathrm{H}), 5.14$ (dd, J 11.0, $1.3 \mathrm{~Hz}, 1 \mathrm{H}), 5.08$ (dd, J 17.7, $1.3 \mathrm{~Hz}, 1 \mathrm{H}), 4.76(\mathrm{~d}, J 11.2 \mathrm{~Hz}$, $1 \mathrm{H}), 4.54(\mathrm{~s}, 2 \mathrm{H}), 4.52(\mathrm{~d}, J=11.1 \mathrm{~Hz}, 1 \mathrm{H}), 3.81(\mathrm{~s}, 3 \mathrm{H}), 3.72-3.67(\mathrm{~m}, 1 \mathrm{H}), 3.63-3.53(\mathrm{~m}, 3 \mathrm{H}), 3.48(\mathrm{dd}, J 11.0$, $5.5 \mathrm{~Hz}, 1 \mathrm{H}), 2.49$ (dd, J 7.2, $5.5 \mathrm{~Hz}, 1 \mathrm{H}), 1.06$ (s, 3H) ppm; ${ }^{13} \mathrm{C} N M R\left(126 \mathrm{MHz}^{\mathrm{CDCl}}\right)_{3} \delta$ 159.4, 140.6, 138.2, $130.6,129.7,128.6,127.8,127.7,114.9,113.9,83.3,73.5,73.2,71.9,68.9,55.4,45.4,18.8$ ppm; HRMS calcd for $\mathrm{C}_{22} \mathrm{H}_{28} \mathrm{O}_{4} \mathrm{Na}\left[\mathrm{M}+\mathrm{Na}^{+}\right]$: 379.1880 , found 379.1887 (1.8 ppm).

(士)-(S)-2-[(S)-2-(Benzyloxy)-1-(4-methoxybenzyloxy)ethyl]-2-methylbut-3-enal (19): Following General Procedure B and purification by flash chromatography using 15:85 EtOAc:Hex provided aldehyde 19 as a clear oil (1.02 g, 94\% yield): $\mathrm{R}_{f}=0.2$ (30:70 EtOAc:Hex); Molecular formula: $\mathrm{C}_{22} \mathrm{H}_{26} \mathrm{O}_{4} ; \mathrm{MW}: 354.45$; IR (neat) $v_{\max }$ $1726 \mathrm{~cm}^{-1}$; ${ }^{1} \mathrm{H}$ NMR $\left(500 \mathrm{MHz}, \mathrm{CDCl}_{3}\right) \delta 9.55(\mathrm{~s}, 1 \mathrm{H}), 7.41-7.29(\mathrm{~m}, 5 \mathrm{H}), 7.25(\mathrm{~d}, J 8.5 \mathrm{~Hz}, 2 \mathrm{H}), 6.89(\mathrm{~d}, J 8.5 \mathrm{~Hz}$, 2H), 6.11 (dd, J 17.8, 10.9 Hz, 1H), 5.33 (d, J $10.9 \mathrm{~Hz}, 1 \mathrm{H}), 5.18$ (d, J $17.7 \mathrm{~Hz}, 1 \mathrm{H}), 4.72$ (d, J $11.2 \mathrm{~Hz}, 1 \mathrm{H}), 4.54(\mathrm{~d}$, J $11.3 \mathrm{~Hz}, 1 \mathrm{H}), 4.52$ (s, 2H), 3.89 (t, J $4.9 \mathrm{~Hz}, 1 \mathrm{H}), 3.82(\mathrm{~s}, 3 \mathrm{H}), 3.62$ (d, J $4.9 \mathrm{~Hz}, 2 \mathrm{H}), 1.22(\mathrm{~s}, 3 \mathrm{H}) ;{ }^{13} \mathrm{C} \mathrm{NMR}(126$ $\left.\mathrm{MHz}_{2} \mathrm{CDCl}_{3}\right) \delta 201.8,159.3,137.9,136.5,130.3,129.6,128.5,127.8,127.7,117.5,113.8,81.2,73.4,73.2,70.3$, $56.2,55.3,15.3$ ppm; HRMS calcd for $\mathrm{C}_{22} \mathrm{H}_{26} \mathrm{O}_{4} \mathrm{Na}\left[\mathrm{M}+\mathrm{Na}^{+}\right]$: 377.1723 , found 377.1726 (0.7 ppm).

( \pm )-(2R,3R)-3-[(S)-2-(Benzyloxy)-1-(4-methoxybenzyloxy)ethyl)-2-hydroxy-3-methylpent-4-enenitrile (20a) and $\quad( \pm)-(2 S, 3 R)-3-[(S)-2-($ benzyloxy)-1-(4-methoxybenzyloxy)ethyl]-2-hydroxy-3-methylpent-4-enenitrile (20b): Cyanohydrins 20a and 20b were prepared following General Procedure C. ${ }^{1} \mathrm{H}$ NMR spectroscopic analysis of the crude reaction indicated an 8:1 mixture of 2,4-syn and anti diastereomers (Table 1, entry 4). Purification by flash chromatography using 25:75 EtOAc:Hex provided 20a and 20b (47.9 mg, 93\% yield) as a clear oil. 20a: $\mathrm{R}_{f}=0.13$ (25:75 EtOAc:Hex); Molecular formula: $\mathrm{C}_{23} \mathrm{H}_{27} \mathrm{NO}_{4} ; \mathrm{MW}: 381.47$; IR (neat) $\mathrm{v}_{\max } 3431$, $2247 \mathrm{~cm}^{-1} ;{ }^{1} \mathrm{H}$ NMR (500 MHz, $\left.\mathrm{CDCl}_{3}\right) \delta 7.40-7.30(\mathrm{~m}, 5 \mathrm{H}), 7.23(\mathrm{~d}, J 8.7 \mathrm{~Hz}, 2 \mathrm{H}), 6.87(\mathrm{~d}, J 8.7 \mathrm{~Hz}, 2 \mathrm{H}), 6.03$ (dd, J 17.6, $11.0 \mathrm{~Hz}, 1 \mathrm{H}), 5.35$ (dd, J 11.0, $0.9 \mathrm{~Hz}, 1 \mathrm{H}), 5.27$ (dd, J 17.6, $0.9 \mathrm{~Hz}, 1 \mathrm{H}), 4.68(\mathrm{~d}, J 11.0 \mathrm{~Hz}, 1 \mathrm{H}), 4.58$ $(\mathrm{d}, J 5.0 \mathrm{~Hz}, 1 \mathrm{H}), 4.55(\mathrm{~s}, 1 \mathrm{H}), 4.54(\mathrm{~s}, 1 \mathrm{H}), 4.46(\mathrm{~d}, J 11.0 \mathrm{~Hz}, 1 \mathrm{H}), 3.84(\mathrm{~d}, J 5.0 \mathrm{~Hz}, 1 \mathrm{H}), 3.81(\mathrm{~s}, 3 \mathrm{H}), 3.69-3.64$ $(\mathrm{m}, 2 \mathrm{H}), 3.61-3.56(\mathrm{~m}, 1 \mathrm{H}), 1.20(\mathrm{~s}, 3 \mathrm{H}) ;{ }^{13} \mathrm{C} \mathrm{NMR}\left(126 \mathrm{MHz}, \mathrm{CDCl}_{3}\right) \delta 159.6,137.3,136.9,129.9,129.8,128.7$, 128.2, 128.0, 118.6, 118.5, 114.0, 82.3, 73.8, 73.2, 69.9, 67.4, 55.4, 48.4, 17.2 ppm; HRMS calcd for $\mathrm{C}_{23} \mathrm{H}_{27} \mathrm{NO}_{4} \mathrm{Na}\left[\mathrm{M}+\mathrm{Na}^{+}\right]$: 404.1832, found 404.1826 (-1.5 ppm). 20b: $\mathrm{R}_{f}=0.17$ (25:75 EtOAc:Hex); IR (neat) $v_{\max }$ 3421, $2244 \mathrm{~cm}^{-1}$; ${ }^{1} \mathrm{H}$ NMR $\left(500 \mathrm{MHz}, \mathrm{CDCl}_{3}\right) \delta 7.41-7.30(\mathrm{~m}, 5 \mathrm{H}), 7.29(\mathrm{~d}, J 8.5 \mathrm{~Hz}, 2 \mathrm{H}), 6.89(\mathrm{~d}, J 8.6 \mathrm{~Hz}, 2 \mathrm{H})$, $6.01(\mathrm{dd}, J 17.6,11.0 \mathrm{~Hz}, 1 \mathrm{H}), 5.30(\mathrm{~d}, J 11.0 \mathrm{~Hz}, 1 \mathrm{H}), 5.23(\mathrm{~d}, J 17.6 \mathrm{~Hz}, 1 \mathrm{H}), 4.82$ (d, J $10.8 \mathrm{~Hz}, 1 \mathrm{H}), 4.58(\mathrm{~d}, J$ $10.8 \mathrm{~Hz}, 1 \mathrm{H}$ ), 4.54 (s, 2H), 4.38 (app.dd, J 8.8, 2.1 Hz, 1H), 3.95 (dd, J 5.7, 3.3 Hz, 1H), 3.92 (app.dd, J 8.8, $2.8 \mathrm{~Hz}$, 1H), 3.81 (s, 3H), 3.62 (dd, J 10.8, 3.4 Hz, 1H), 3.58 (dd, J 10.8, 5.6 Hz, 1H), 1.21 (s, 3H) ppm; ${ }^{13} \mathrm{C}$ NMR (100.6 $\left.\mathrm{MHz}_{2} \mathrm{CDCl}_{3}\right) \delta 159.7,137.9,136.0,130.3,129.6,128.6,128.0,127.7,119.0,117.9,114.2,82.8,73.6,73.5,71.7$, 69.5, 55.4, 46.9, 17.3 ppm; HRMS calcd for $\mathrm{C}_{23} \mathrm{H}_{27} \mathrm{NO}_{4} \mathrm{Na}\left[\mathrm{M}+\mathrm{Na}^{+}\right]$: 404.1832 , found 404.1827 (-1.2 ppm).

(士)-(S)-2-[(S)-2-(Benzyloxy)-1-hydroxyethyl]-2-methylbut-3-enal (21): To a solution of racemic aldehyde 6 (0.14 g, $0.42 \mathrm{mmol} 1.0$ equiv.) in dry THF $(4.1 \mu \mathrm{L}, 0.10 \mathrm{M})$ at $0{ }^{\circ} \mathrm{C}$, HF-pyridine $(0.84 \mathrm{~mL}, 0.84 \mathrm{mmol}, 2.0$ $\mathrm{mL} / \mathrm{mmol}$ ) was added. The solution was warmed to room temperature and stirred overnight. An aqueous solution of $\mathrm{NaHCO}_{3}$ was added and the aqueous layer was extracted with $\mathrm{Et}_{2} \mathrm{O}(3 \mathrm{x})$. The organic layers were combined, dried with $\mathrm{MgSO}_{4}$, filtered and concentrated in vacuo. Purification by flash chromatography using 30:70 EtOAc:Hex provided aldehyde 21 as a clear oil (56.6 mg, 58\% yield): $R_{f}=0.23$ (30:70 EtOAc:Hex); Molecular formula: $\mathrm{C}_{14} \mathrm{H}_{18} \mathrm{O}_{3} ; \mathrm{MW}$ : 234.30; IR (neat) $v_{\max } 3460,1725 \mathrm{~cm}^{-1} ;{ }^{1} \mathrm{H} \mathrm{NMR}\left(500 \mathrm{MHz}, \mathrm{CDCl}_{3}\right) \delta 9.53$ (s, 1H), $7.38-7.28(\mathrm{~m}, 5 \mathrm{H}), 6.07$ (dd, J 17.7, $10.9 \mathrm{~Hz}, 1 \mathrm{H}), 5.39$ (d, J $10.9 \mathrm{~Hz}, 1 \mathrm{H}), 5.21$ (d, J $17.8 \mathrm{~Hz}, 1 \mathrm{H}), 4.53$ (s, 2H), 4.08 (dd, J 7.3, 3.7 Hz, 1H), $3.54(\mathrm{dd}, J$ 9.8, 3.7 Hz, 1H), $3.48(\mathrm{dd}, J 9.8,7.4 \mathrm{~Hz}, 1 \mathrm{H}), 2.63(\mathrm{~s}, 1 \mathrm{H}), 1.21(\mathrm{~s}, 3 \mathrm{H})$

ppm; ${ }^{13} \mathrm{C}$ NMR $\left(100.6 \mathrm{MHz}, \mathrm{CDCl}_{3}\right) \delta 202.3,137.7,135.5,128.5,127.9,127.8,118.4,73.8,73.5,70.7,55.7$, 15.1 ppm; HRMS for $\mathrm{C}_{14} \mathrm{H}_{18} \mathrm{O}_{3} \mathrm{Na}\left[\mathrm{M}+\mathrm{Na}^{+}\right]: 257.1148$, found 257.1146 (-0.9 ppm). 
$( \pm)-(2 R, 3 R)-3-[(S)-2-(B e n z y l o x y)-1-h y d r o x y e t h y l]-2-h y d r o x y-3-m e t h y l p e n t-4-e n e n i t r i l e ~(22 a)$ and $( \pm)-(2 S, 3 R)-$ 3-((S)-2-(benzyloxy)-1-hydroxyethyl)-2-hydroxy-3-methylpent-4-enenitrile (22b): Cyanohydrins 22a and 22b were prepared following General Procedure C. ${ }^{1} \mathrm{H}$ NMR spectroscopic analysis of the crude reaction indicated a 1:4 mixture of 2,4-syn and anti diastereomers (Table 1, entry 5). Purification by flash chromatography using 50:50 EtOAc:Hex provided 22a and 22b (26 mg, 89\% yield). 22a: $\mathrm{R}_{f}=0.33$ (50:50 EtOAc:Hex); Molecular formula: $\mathrm{C}_{15} \mathrm{H}_{19} \mathrm{NO}_{3}$; MW: 261.32; IR (neat) $v_{\max } 3428,2246 \mathrm{~cm}^{-1} ;{ }^{1} \mathrm{H} \mathrm{NMR}\left(500 \mathrm{MHz}, \mathrm{CDCl}_{3}\right) \delta 7.41-7.29$ (m, 5H), 6.11 (dd, J 17.7, 11.0 Hz, 1H), $5.43(\mathrm{dd}, J 11.0,0.8 \mathrm{~Hz}, 1 \mathrm{H}), 5.30(\mathrm{dd}, J 17.7,0.9 \mathrm{~Hz}, 1 \mathrm{H}), 4.66(\mathrm{~d}, J 4.5 \mathrm{~Hz}$, $1 \mathrm{H}), 4.57(\mathrm{~d}, J 11.7 \mathrm{~Hz}, 1 \mathrm{H}), 4.54(\mathrm{~d}, J 11.7 \mathrm{~Hz}, 1 \mathrm{H}), 3.97$ (ddd, J 8.1, 3.9, $2.3 \mathrm{~Hz}, 1 \mathrm{H}), 3.72(\mathrm{~d}, J 4.5,1 \mathrm{H}), 3.58$ (dd, J 9.7, $3.8 \mathrm{~Hz}, 1 \mathrm{H}), 3.46(\mathrm{dd}, J$ 9.7, $8.1 \mathrm{~Hz}, 1 \mathrm{H}), 2.76(\mathrm{~d}, J 2.3 \mathrm{~Hz}, 1 \mathrm{H}), 1.17(\mathrm{~s}, 3 \mathrm{H}) \mathrm{ppm} ;{ }^{13} \mathrm{C} \mathrm{NMR}(100.6 \mathrm{MHz}$, $\left.\mathrm{CDCl}_{3}\right) \delta 137.2,135.6,128.8,128.4,128.0,119.2,118.2,75.0,73.8,70.4,68.4,46.9,16.1$ ppm; HRMS for $\mathrm{C}_{15} \mathrm{H}_{20} \mathrm{O}_{3} \mathrm{~N}\left[\mathrm{M}+\mathrm{H}^{+}\right]$: 262.1438, found 262.1438 (0.3 ppm). 22b: $R_{f}=0.29$ (50:50 EtOAc:Hex); IR (neat) U max 3423, $2246 \mathrm{~cm}^{-1}$; ${ }^{1} \mathrm{H}$ NMR $\left(500 \mathrm{MHz}, \mathrm{CDCl}_{3}\right) \delta 7.42-7.28(\mathrm{~m}, 5 \mathrm{H}), 6.15$ (dd, J 17.7, $\left.11.0 \mathrm{~Hz}, 1 \mathrm{H}\right), 5.35(\mathrm{~d}, J 11.0 \mathrm{~Hz}$, $1 \mathrm{H}), 5.25(\mathrm{dd}, J 17.7,0.8 \mathrm{~Hz}, 1 \mathrm{H}), 4.54(\mathrm{~s}, 2 \mathrm{H}), 4.41(\mathrm{~d}, J 8.0 \mathrm{~Hz}, 1 \mathrm{H}), 4.33(\mathrm{~d}, J 9.0 \mathrm{~Hz}, 1 \mathrm{H}), 4.22(\mathrm{dd}, J 9.1,2.9 \mathrm{~Hz}$, 1H), 3.53 (dd, J 9.6, $3.0 \mathrm{~Hz}, 1 \mathrm{H}$ ), 3.37 (app.t, J $9.3 \mathrm{~Hz}, 1 \mathrm{H}), 3.03$ (s, 1H), 1.15 (s, 3H) ppm; ${ }^{13} \mathrm{C} \mathrm{NMR} \mathrm{(126} \mathrm{MHz,}$ $\left.\mathrm{CDCl}_{3}\right) \delta 137.4,135.7,128.8,128.2,127.9,118.7,117.9,74.6,73.7,70.6,70.5,45.4,16.7$ ppm; HRMS for $\mathrm{C}_{15} \mathrm{H}_{19} \mathrm{O}_{3} \mathrm{NNa}\left[\mathrm{M}+\mathrm{Na}^{+}\right]: 284.1257$, found 284.1260 (0.9 ppm).

(士)-(S)-Methyl 2-((S)-2,2,3,3,9,9-hexamethyl-8,8-diphenyl-4,7-dioxa-3,8-disiladecan-5-yl)-2-methylbut-3-enoate (S7): To a solution of $(S)$-methyl 2-((S)-2-((tert-butyldiphenylsilyl)oxy)-1-hydroxyethyl)-2-methylbut-3enoate ${ }^{22}\left(1.0 \mathrm{~g}, 2.5 \mathrm{mmol}, 1.0\right.$ equiv.) in anhydrous $\mathrm{CH}_{2} \mathrm{Cl}_{2}(25 \mathrm{~mL}, 0.10 \mathrm{M})$ at $0{ }^{\circ} \mathrm{C}, 2,6$-lutidine $(0.59 \mathrm{~mL}, 5.1$ mmol, 2.0 equiv.) and TBSOTf ( $0.84 \mathrm{~mL}, 3.5 \mathrm{mmol}, 1.4$ equiv.) were added. The solution was stirred until the alcohol was consumed, 4 hours at $0{ }^{\circ} \mathrm{C}$, as determined by TLC. An aqueous solution of $\mathrm{NH}_{4} \mathrm{Cl}$ was added and the aqueous layer was extracted with $\mathrm{Et}_{2} \mathrm{O}(3 \times)$. The organic layers were combined, dried over $\mathrm{MgSO}_{4}$, filtered and concentrated in vacuo. Purification by flash chromatography using 5:95 EtOAc:Hex provided protected ester S7 as a clear oil (1.02 g, 76\% yield): $\mathrm{R}_{f}=0.35$ (5:95 EtOAc:Hex); Molecular formula: $\mathrm{C}_{30} \mathrm{H}_{46} \mathrm{O}_{4} \mathrm{Si}_{2} ; \mathrm{MW}$ : 526.86; IR (neat) $v_{\max } 1737 \mathrm{~cm}^{-1} ;{ }^{1} \mathrm{H}$ NMR $\left(500 \mathrm{MHz}, \mathrm{CDCl}_{3}\right) \delta 7.68-7.62(\mathrm{~m}, 4 \mathrm{H}), 7.45-7.35$ (m, $\left.6 \mathrm{H}\right), 6.09$ (dd, J 17.7, $10.8 \mathrm{~Hz}, 1 \mathrm{H}$ ), 5.14 (dd, J 10.8, $1.0 \mathrm{~Hz}, 1 \mathrm{H}$ ), 5.04 (dd, J 17.7, 1.1 Hz, 1H), 4.19 (t, J $5.5 \mathrm{~Hz}, 1 \mathrm{H}$ ), $3.57(\mathrm{~s}, 3 \mathrm{H})$, 3.55-3.59 (m, 1H), $3.47(\mathrm{dd}, J$ 10.9, $5.2 \mathrm{~Hz}, 1 \mathrm{H}), 1.25(\mathrm{~s}, 3 \mathrm{H}), 1.04(\mathrm{~s}, 9 \mathrm{H}), 0.79(\mathrm{~s}, 9 \mathrm{H}), 0.02(\mathrm{~s}, 3 \mathrm{H}),-0.08(\mathrm{~s}, 3 \mathrm{H})$; ${ }^{13} \mathrm{C}$ NMR $\left(126 \mathrm{MHz}, \mathrm{CDCl}_{3}\right) \delta 175.1,139.2,135.84,135.79,133.4,133.3,129.84,129.82,127.815,127.807$, 114.9, 77.6, 66.4, 53.8, 52.0, 27.2, 25.9, 19.3, 18.2, 16.3, -3.9, -5.0 ppm; HRMS calcd for $\mathrm{C}_{30} \mathrm{H}_{47} \mathrm{O}_{4} \mathrm{Si}_{2}\left[\mathrm{M}+\mathrm{H}^{+}\right]$: 527.3007 , found 527.3010 (0.6 ppm).

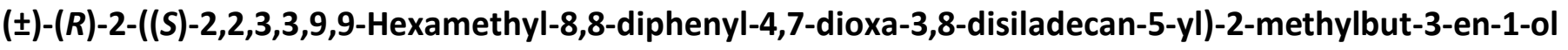
(S8): Following General Procedure A and purification by flash chromatography using 5:95 EtOAc:Hex, primary alcohol S8 was obtained as a clear oil $\left(0.73 \mathrm{~g}, 97 \%\right.$ yield): $\mathrm{R}_{f}=0.24$ (5:95 EtOAc:Hex); Molecular formula: $\mathrm{C}_{29} \mathrm{H}_{46} \mathrm{O}_{3} \mathrm{Si}_{2}$; MW: 498.85; IR (neat) $v_{\max } 3454,2955,2932,1111 \mathrm{~cm}^{-1} ;{ }^{1} \mathrm{H} \mathrm{NMR}\left(500 \mathrm{MHz}, \mathrm{CDCl}_{3}\right) \delta 7.69-7.63$ (m, 4H), $7.48-7.35(\mathrm{~m}, 6 \mathrm{H}), 5.84$ (dd, J 17.7, $11.0 \mathrm{~Hz}, 1 \mathrm{H}), 5.05$ (dd, J 11.0, $1.3 \mathrm{~Hz}, 1 \mathrm{H}$ ), 5.00 (dd, J 17.7, 1.4 Hz, $1 \mathrm{H}), 3.73$ (dd, J 10.9, $5.5 \mathrm{~Hz}, 1 \mathrm{H}$ ), 3.67 (dd, J 5.6, $4.2 \mathrm{~Hz}, 1 \mathrm{H}), 3.65-3.62(\mathrm{~m}, 1 \mathrm{H}), 3.54(\mathrm{dd}, J 10.8,4.0 \mathrm{~Hz}, 1 \mathrm{H})$, 3.49 (dd, J 10.9, 6.2 Hz, 1H), $2.65(t, J 6.3 \mathrm{~Hz}, 1 \mathrm{H}), 1.06(\mathrm{~s}, 9 \mathrm{H}), 1.05(\mathrm{~s}, 3 \mathrm{H}), 0.83(\mathrm{~s}, 9 \mathrm{H}), 0.04(\mathrm{~s}, 3 \mathrm{H}),-0.11(\mathrm{~s}$, $3 \mathrm{H}) \mathrm{ppm} ;{ }^{13} \mathrm{C}$ NMR $\left(126 \mathrm{MHz}, \mathrm{CDCl}_{3}\right) \delta 141.7,135.92,135.85,133.1,133.0,129.952,129.951,127.88,127.85$, 114.5, 79.1, 68.3, 66.5, 46.2, 27.0, 26.0, 19.2, 18.247, 18.246, -4.0, -4.9 ppm; HRMS calcd for $\mathrm{C}_{29} \mathrm{H}_{47} \mathrm{O}_{3} \mathrm{Si}_{2}$ $\left[\mathrm{M}+\mathrm{H}^{+}\right]:$499.3058, found $499.3062(0.7 \mathrm{ppm})$.

(士)-(S)-2-((S)-2,2,3,3,9,9-hexamethyl-8,8-diphenyl-4,7-dioxa-3,8-disiladecan-5-yl)-2-methylbut-3-enal

(23):

Following General Procedure B and purification by flash chromatography using 5:95 EtOAc:Hex provided aldehyde 21 as a clear oil (0.53 g, 75\% yield): $\mathrm{R}_{f}=0.30$ (5:95 EtOAc:Hex); Molecular formula: $\mathrm{C}_{29} \mathrm{H}_{44} \mathrm{O}_{3} \mathrm{Si}_{2} ; \mathrm{MW}$ 496.84; IR (neat) $v_{\max } 1727 \mathrm{~cm}^{-1}$; ${ }^{1} \mathrm{H}$ NMR $\left(500 \mathrm{MHz}, \mathrm{CDCl}_{3}\right) \delta 9.68(\mathrm{~s}, 1 \mathrm{H})$, 7.38-7.67 (m, 10H), 6.06 (dd, J 10.9, 
$17.6 \mathrm{~Hz}, 1 \mathrm{H}), 5.29$ (dd, J 0.7, $11.0 \mathrm{~Hz}, 1 \mathrm{H}), 5.14(\mathrm{dd}, J 0.7,17.8 \mathrm{~Hz}, 1 \mathrm{H}), 4.01(\mathrm{dd}, J 4.5,6.5 \mathrm{~Hz}, 1 \mathrm{H}), 3.58(\mathrm{dd}, J$ 6.6, $10.7 \mathrm{~Hz}, 1 \mathrm{H}), 3.53$ (dd, J 4.5, $10.9 \mathrm{~Hz}, 1 \mathrm{H}), 1.20(\mathrm{~s}, 3 \mathrm{H}), 1.04(\mathrm{~s}, 9 \mathrm{H}), 0.81(\mathrm{~s}, 9 \mathrm{H}), 0.01(\mathrm{~s}, 3 \mathrm{H}),-0.15(\mathrm{~s}, 3 \mathrm{H})$; ${ }^{13} \mathrm{C} \mathrm{NMR}\left(126 \mathrm{MHz}, \mathrm{CDCl}_{3}\right) \delta 201.7,137.3,135.83,135.79,133.0,132.9,129.96,129.95,127.89,127.87,117.1$, 76.7, 65.5, 56.9, 26.9, 25.9, 19.2, 18.2, 14.4, -4.1, -4.9 ppm; HRMS calcd for $\mathrm{C}_{29} \mathrm{H}_{44} \mathrm{O}_{3} \mathrm{Si}{ }_{2} \mathrm{Na}\left[\mathrm{M}+\mathrm{Na}^{+}\right]: 519.2721$, found $519.2724(0.5 \mathrm{ppm})$.

( \pm )-(2R,3R)-3-((S)-2,2,3,3,9,9-hexamethyl-8,8-diphenyl-4,7-dioxa-3,8-disiladecan-5-yl)-2-hydroxy-3-methylpent-4-enenitrile (24a) and $( \pm)-(2 S, 3 R)-3-((S)-2,2,3,3,9,9-h e x a m e t h y l-8,8-d i p h e n y l-4,7-d i o x a-3,8-d i s i l a d e c a n-$ 5-yl)-2-hydroxy-3-methylpent-4-enenitrile (24b): Cyanohydrins 24a and 24b were prepared following General Procedure C. ${ }^{1} \mathrm{H}$ NMR spectroscopic analysis of the crude reaction indicated a 1.5:1 mixture of 2,4-syn and anti diastereomers (Table 1, entry 6). Purification by flash chromatography using 25:75 $\mathrm{Pr}_{2} \mathrm{O}: \mathrm{Hex}$ provided 24a and 24b (67 mg, 93\% yield) as clear oils. 24a: $\mathrm{R}_{f}=0.29$ (25:75 $i \mathrm{Pr}_{2} \mathrm{O}: \mathrm{Hex}$ ); Molecular formula: $\mathrm{C}_{30} \mathrm{H}_{45} \mathrm{NO}_{3} \mathrm{Si}_{2}$; MW: 523.86; IR (neat) $v_{\max } 3448,2249 \mathrm{~cm}^{-1} ;{ }^{1} \mathrm{H}$ NMR $\left(500 \mathrm{MHz}, \mathrm{CDCl}_{3}\right) \delta 7.72-7.62(\mathrm{~m}, 4 \mathrm{H}), 7.52-7.36$ (m, $6 \mathrm{H}), 5.98(\mathrm{dd}, J 17.6,11.0 \mathrm{~Hz}, 1 \mathrm{H}), 5.30(\mathrm{dd}, J 10.9,0.9 \mathrm{~Hz}, 1 \mathrm{H}), 5.23(\mathrm{dd}, J 17.6,0.9 \mathrm{~Hz}, 1 \mathrm{H}), 4.53(\mathrm{~d}, J 6.6 \mathrm{~Hz}$, $1 \mathrm{H}), 4.25(\mathrm{~d}, J 6.6 \mathrm{~Hz}, 1 \mathrm{H}), 3.87(\mathrm{dd}, J 6.9,3.6 \mathrm{~Hz}, 1 \mathrm{H}), 3.64(\mathrm{dd}, J 11.3,6.9 \mathrm{~Hz}, 1 \mathrm{H}), 3.52(\mathrm{dd}, J 11.3,3.6 \mathrm{~Hz}, 1 \mathrm{H})$, $1.16(\mathrm{~s}, 3 \mathrm{H}), 1.08(\mathrm{~s}, 9 \mathrm{H}), 0.78(\mathrm{~s}, 9 \mathrm{H}), 0.01(\mathrm{~s}, 3 \mathrm{H}),-0.25(\mathrm{~s}, 3 \mathrm{H}) \mathrm{ppm} ;{ }^{13} \mathrm{C} \mathrm{NMR}\left(126 \mathrm{MHz}, \mathrm{CDCl}_{3}\right) \delta 138.4,135.90$, $135.88,132.13,132.09,130.34,130.30,128.11,128.08,118.8,118.0,76.7,67.1,65.6,49.5,27.0,26.0,19.2$, 18.2, 15.3, -4.2, -5.0 ppm; HRMS calcd for $\mathrm{C}_{30} \mathrm{H}_{46} \mathrm{NO}_{3} \mathrm{Si}_{2}\left[\mathrm{M}+\mathrm{H}^{+}\right]: 524.3011$, found 524.3011 (0.07 ppm). 24b : $\mathrm{R}_{f}$ $=0.21$ (25:75 $\left.\mathrm{iPr}_{2} \mathrm{O}: \mathrm{Hex}\right)$; IR (neat) $\mathrm{v}_{\max } 3423,2247 \mathrm{~cm}^{-1} ;{ }^{1} \mathrm{H} \mathrm{NMR}\left(500 \mathrm{MHz}, \mathrm{CDCl}_{3}\right) \delta 7.67-7.61(\mathrm{~m}, 4 \mathrm{H}), 7.49-$ $7.35(\mathrm{~m}, 6 \mathrm{H}), 5.83$ (dd, J 17.6, $11.0 \mathrm{~Hz}, 1 \mathrm{H}), 5.28$ (dd, J 10.9, $0.8 \mathrm{~Hz}, 1 \mathrm{H}), 5.22$ (dd, J 17.6, $0.8 \mathrm{~Hz}, 1 \mathrm{H}), 4.77(\mathrm{~d}, J$ $6.2 \mathrm{~Hz}, 1 \mathrm{H}), 3.83(\mathrm{dd}, J$ 5.5, $3.9 \mathrm{~Hz}, 1 \mathrm{H}), 3.79(\mathrm{~d}, J 6.2 \mathrm{~Hz}, 1 \mathrm{H}), 3.66(\mathrm{dd}, J 11.3,5.5 \mathrm{~Hz}, 1 \mathrm{H}), 3.58$ (dd, J $11.3,3.9$ $\mathrm{Hz}, 1 \mathrm{H}), 1.22(\mathrm{~s}, 3 \mathrm{H}), 1.07(\mathrm{~s}, 9 \mathrm{H}), 0.84(\mathrm{~s}, 9 \mathrm{H}), 0.09(\mathrm{~s}, 3 \mathrm{H}),-0.09(\mathrm{~s}, 3 \mathrm{H}) \mathrm{ppm} ;{ }^{13} \mathrm{C} \mathrm{NMR}\left(126 \mathrm{MHz}^{\mathrm{CDCl}}\right)_{3} \delta$ 137.0, 135.93, 135.85, 132.8, 132.7, 130.13, 130.12, 128.00, 127.95, 118.9, 118.4, 78.4, 68.2, 66.0, 47.9, 27.0, 26.0, 19.2, 18.2, 16.2, -4.2, -5.0 ppm; HRMS calcd for $\mathrm{C}_{30} \mathrm{H}_{46} \mathrm{NO}_{3} \mathrm{Si}_{2}\left[\mathrm{M}+\mathrm{H}^{+}\right]$: 524.3011, found $524.3007(-0.8$ $\mathrm{ppm})$.

( \pm )-(R)-Methyl 3-hydroxy-2,2-dimethyl-6-phenylhexanoate (S9): To a solution of 4-phenylbutanal ${ }^{43}$ (2.4 g, 16 mmol, 1.0 equiv.) in anhydrous $\mathrm{CH}_{2} \mathrm{Cl}_{2}(160 \mathrm{ml}, 0.10 \mathrm{M})$ at $-40^{\circ} \mathrm{C}, \mathrm{BF}_{3} \cdot \mathrm{OEt}_{2}(3.0 \mathrm{ml}, 24 \mathrm{mmol}, 1.5$ equiv.) was added. A solution of the commercially available enoxysilane ((1-methoxy-2-methylprop-1-en-1yl)oxy)trimethylsilane) $\left(6.5 \mathrm{ml}, 32 \mathrm{mmol}, 2.0\right.$ equiv.) in $\mathrm{CH}_{2} \mathrm{Cl}_{2}$ was immediately added at the same temperature. The resulting solution was stirred until the aldehyde was completely consumed as indicated by TLC (one hour). A saturated aqueous solution of $\mathrm{NH}_{4} \mathrm{Cl}$ was added and the aqueous layer was extracted with $\mathrm{Et}_{2} \mathrm{O}(3 \times)$. The organic layers were combined, washed with brine, dried over $\mathrm{MgSO}_{4}$, filtered and concentrated in vacuo. Purification by flash chromatography using 25:75 EtOAc:Hex provided ester S9 as a clear oil (quantitative yield after 2 steps, $4.0 \mathrm{~g}$ ): $\mathrm{R}_{f}=0.25$ (25:75 EtOAc:Hex); Molecular formula: $\mathrm{C}_{15} \mathrm{H}_{22} \mathrm{O}_{3} ; \mathrm{MW}: 250.34$; IR (neat) $V_{\max } 3496,1729 \mathrm{~cm}^{-1}{ }^{1}{ }^{1} \mathrm{H} N M R\left(500 \mathrm{MHz}, \mathrm{CDCl}_{3}\right) \delta 7.32-7.14(\mathrm{~m}, 5 \mathrm{H}), 3.69(\mathrm{~s}, 3 \mathrm{H}), 3.64$ (ddd, J 10.6, 6.9, $2.1 \mathrm{~Hz}, 1 \mathrm{H}), 2.65(\mathrm{~m}, 2 \mathrm{H}), 2.41(\mathrm{~d}, \mathrm{~J} 6.9 \mathrm{~Hz}, 1 \mathrm{H}), 2.04-1.88(\mathrm{~m}, 1 \mathrm{H}), 1.75-1.61(\mathrm{~m}, 1 \mathrm{H}), 1.49(\mathrm{~m}, 1 \mathrm{H}), 1.39$ $-1.28(\mathrm{~m}, 1 \mathrm{H}), 1.18(\mathrm{~s}, 3 \mathrm{H}), 1.16(\mathrm{~s}, 3 \mathrm{H}) ;{ }^{13} \mathrm{C} \mathrm{NMR}\left(100.6 \mathrm{MHz}, \mathrm{CDCl}_{3}\right) \delta 178.6,142.7,128.7,128.6,126.0,76.8$, 52.3, 47.5, 36.0, 31.5, 28.7, 22.7, 20.7; HRMS calcd for $\mathrm{C}_{15} \mathrm{H}_{22} \mathrm{O}_{3} \mathrm{Na}\left[\mathrm{M}+\mathrm{Na}^{+}\right]$: 273.1461, found $273.1458(-1.1$ $\mathrm{ppm})$.

( \pm )-(R)-Methyl 3-(tert-butyldimethylsilyloxy)-2,2-dimethyl-6-phenylhexanoate (S10): To a solution of secondary alcohol $\mathbf{S 9}\left(0.88 \mathrm{~g}, 3.5 \mathrm{mmol}, 1.0\right.$ equiv.) in anhydrous $\mathrm{CH}_{2} \mathrm{Cl}_{2}(35 \mathrm{~mL}, 0.10 \mathrm{M})$ at $0{ }^{\circ} \mathrm{C}$, pyridine $(0.56$ $\mathrm{mL}, 7.0 \mathrm{mmol}, 2.0$ equiv.) and TBSOTf ( $1.1 \mathrm{~mL}, 4.9 \mathrm{mmol}, 1.4$ equiv.) were added. The solution was stirred until the alcohol was consumed, 2.5 hours at $0{ }^{\circ} \mathrm{C}$, as determined by TLC. An aqueous solution of $\mathrm{NH}_{4} \mathrm{Cl}$ was added and the aqueous layer extracted with $\mathrm{Et}_{2} \mathrm{O}(3 \times)$. The organic layers were combined, dried over $\mathrm{MgSO}_{4}$, filtered and concentrated in vacuo. Purification by flash chromatography using 5:95 EtOAc:Hex provided protected 
ester S10: $\mathrm{R}_{f}=0.25$ (5:95 EtOAc:Hex); Molecular formula: $\mathrm{C}_{21} \mathrm{H}_{36} \mathrm{O}_{3} \mathrm{Si}$; MW: 364.60; IR (neat) $\mathrm{V}_{\max } 1735 \mathrm{~cm}^{-1} ;{ }^{1} \mathrm{H}$ NMR (500 MHz, CDCl $) \delta 7.34-7.27(\mathrm{~m}, 2 \mathrm{H}), 7.24-7.17(\mathrm{~m}, 3 \mathrm{H}), 3.97(\mathrm{dd}, J 6.5,4.1 \mathrm{~Hz}, 1 \mathrm{H}), 3.65(\mathrm{~s}, 3 \mathrm{H}), 2.72$ $-2.52(\mathrm{~m}, 2 \mathrm{H}), 1.90-1.78(\mathrm{~m}, 1 \mathrm{H}), 1.68-1.56(\mathrm{~m}, 1 \mathrm{H}), 1.55-1.42(\mathrm{~m}, 2 \mathrm{H}), 1.22(\mathrm{~s}, 3 \mathrm{H}), 1.13(\mathrm{~s}, 3 \mathrm{H}), 0.94(\mathrm{~s}$, 9H), 0.10 (s, 3H), 0.08 (s, 3H) ppm; $\left.{ }^{13} \mathrm{C} \mathrm{NMR} \mathrm{(100.6} \mathrm{MHz,} \mathrm{CDCl}_{3}\right) \delta$ 177.8, 142.4, 128.6, 128.5, 126., 77.2, 51.8, 48.6, 36.4, 33.9, 29.1, 26.3, 22.4, 20.2, 18.6, -3.4, -4.0 ppm; HRMS for $\mathrm{C}_{21} \mathrm{H}_{37} \mathrm{O}_{3} \mathrm{Si}\left[\mathrm{M}+\mathrm{H}^{+}\right]: 365.2506$, found 365.2508 (0.4 ppm).

( \pm )-(R)-3-(tert-Butyldimethylsilyloxy)-2,2-dimethyl-6-phenylhexan-1-ol (S11): Following General Procedure A and purification by flash chromatography using 10:90 EtOAc:Hex, primary alcohol S11 was obtained as a clear oil (0.7 g, 60\% yield over two steps): $\mathrm{R}_{f}=0.2$ (10:90 EtOAc:Hex); Molecular formula: $\mathrm{C}_{20} \mathrm{H}_{36} \mathrm{O}_{2} \mathrm{Si}$; MW: 336.59; IR (neat) $v_{\max } 3443,2929,2857,1472 \mathrm{~cm}^{-1} ;{ }^{1} \mathrm{H}$ NMR $\left(500 \mathrm{MHz}, \mathrm{CDCl}_{3}\right) \delta 7.31-7.25(\mathrm{~m}, 2 \mathrm{H}), 7.22-7.11(\mathrm{~m}, 3 \mathrm{H})$, $3.71(\mathrm{dd}, J 10.8,3.0 \mathrm{~Hz}, 1 \mathrm{H}), 3.51(\mathrm{dd}, J 6.0,3.8 \mathrm{~Hz}, 1 \mathrm{H}), 3.25(\mathrm{dd}, J 10.7,7.6 \mathrm{~Hz}, 1 \mathrm{H}), 2.86(\mathrm{dd}, J 7.6,3.1 \mathrm{~Hz}, 1 \mathrm{H})$, $2.68-2.54(\mathrm{~m}, 2 \mathrm{H}), 1.90-1.80(\mathrm{~m}, 1 \mathrm{H}), 1.79-1.65(\mathrm{~m}, 1 \mathrm{H}), 1.67-1.59(\mathrm{~m}, 1 \mathrm{H}), 1.58-1.47(\mathrm{~m}, 1 \mathrm{H}), 1.03(\mathrm{~s}$, $3 \mathrm{H}), 0.91(\mathrm{~s}, 9 \mathrm{H}), 0.77$ (s, 3H), $0.10(\mathrm{~s}, 3 \mathrm{H}), 0.05(\mathrm{~s}, 3 \mathrm{H}) \mathrm{ppm} ;{ }^{13} \mathrm{C} \mathrm{NMR}\left(100.6 \mathrm{MHz}, \mathrm{CDCl}_{3}\right) \delta$ 142.3, 128.5, 128.4, 125.9, 80.9, 70.4, 39.5, 36.5, 33.3, 29.4, 26.2, 23.9, 21.9, 18.3, -3.7, -4.1 ppm; HRMS for $\mathrm{C}_{20} \mathrm{H}_{37} \mathrm{O}_{2} \mathrm{Si}\left[\mathrm{M}+\mathrm{H}^{+}\right]: 337.2557$, found 337.2561 (1.0 ppm).

( \pm )-(R)-3-(tert-Butyldimethylsilyloxy)-2,2-dimethyl-6-phenylhexanal (25): Following General Procedure B and purification by flash chromatography using 5:95 EtOAc:Hex provided aldehyde 25 as a clear oil $(0.69 \mathrm{~g}, 99 \%$ yield): $\mathrm{R}_{f}=0.21$ (5:95 EtOAc:Hex); Molecular formula: $\mathrm{C}_{20} \mathrm{H}_{34} \mathrm{O}_{2} \mathrm{Si}$; MW: 334.58; IR (neat) $\mathrm{v}_{\max } 1728 \mathrm{~cm}^{-1} ;{ }^{1} \mathrm{H}$ NMR $\left(500 \mathrm{MHz}, \mathrm{CDCl}_{3}\right) \delta 9.62(\mathrm{~s}, 1 \mathrm{H}), 7.32-7.25(\mathrm{~m}, 2 \mathrm{H}), 7.23-7.13(\mathrm{~m}, 3 \mathrm{H}), 3.80-3.77(\mathrm{~m}, 1 \mathrm{H}), 2.67-2.53$ $(\mathrm{m}, 2 \mathrm{H}), 1.82-1.71(\mathrm{~m}, 1 \mathrm{H}), 1.67-1.58(\mathrm{~m}, 1 \mathrm{H}), 1.58-1.45(\mathrm{~m}, 2 \mathrm{H}), 1.05(\mathrm{~s}, 3 \mathrm{H}), 1.02(\mathrm{~s}, 3 \mathrm{H}), 0.90(\mathrm{~s}, 9 \mathrm{H})$, 0.06 (s, 3H), 0.05 (s, 3H) ppm; ${ }^{13} \mathrm{C}$ NMR $\left(100.6 \mathrm{MHz}, \mathrm{CDCl}_{3}\right) \delta 206.7,142.0,128.44,128.43,125.9,76.8,51.4$, $36.3,33.3,28.3,26.0,19.6,18.3,17.9,-3.6,-4.2$ ppm; HRMS for $\mathrm{C}_{20} \mathrm{H}_{33} \mathrm{O}_{2} \mathrm{Si}\left[\mathrm{M}-\mathrm{H}^{+}\right]$: 333.2255, found 333.2248 (1.2 ppm).

( \pm )-(2R,4R)-4-(tert-Butyldimethylsilyloxy)-2-hydroxy-3,3-dimethyl-7-phenylheptanenitrile (26a) and ( \pm )-(2S,4R)-4-(tert-butyldimethylsilyloxy)-2-hydroxy-3,3-dimethyl-7-phenylheptanenitrile (26b): Cyanohydrins 26a and 26b were prepared following General Procedure C. ${ }^{1} \mathrm{H}$ NMR spectroscopic analysis of the crude reaction indicated a 2.5:1 mixture of 2,4-syn and anti diastereomers (Table 1, entry 7). Purification by flash chromatography using 5:95 EtOAc:Hex provided 26a and 26b (63 mg, 82\% yield) as a clear oil. $26 \mathrm{a}: \mathrm{R}_{f}=0.28$ (15:85 EtOAc:Hex); Molecular formula: $\mathrm{C}_{21} \mathrm{H}_{35} \mathrm{NO}_{2} \mathrm{Si}$; MW: 361.60; IR (neat) $\mathrm{V}_{\max } 3446,2246 \mathrm{~cm}^{-1} ;{ }^{1} \mathrm{H}$ NMR (500 $\left.\mathrm{MHz}_{\mathrm{CDCl}}\right) \delta 7.31-7.27(\mathrm{~m}, 2 \mathrm{H}), 7.22-7.14(\mathrm{~m}, 3 \mathrm{H}), 4.40(\mathrm{~d}, J 6.5 \mathrm{~Hz}, 1 \mathrm{H}), 3.60(\mathrm{dd}, J 5.7,3.3 \mathrm{~Hz}, 1 \mathrm{H}), 3.34$ $(\mathrm{d}, J 6.4 \mathrm{~Hz}, 1 \mathrm{H}), 2.68-2.55(\mathrm{~m}, 2 \mathrm{H}), 1.90-1.78(\mathrm{~m}, 2 \mathrm{H}), 1.70-1.48(\mathrm{~m}, 2 \mathrm{H}), 1.05(\mathrm{~s}, 3 \mathrm{H}), 1.04(\mathrm{~s}, 3 \mathrm{H}), 0.89(\mathrm{~s}$, $9 \mathrm{H}), 0.11(\mathrm{~s}, 3 \mathrm{H}), 0.06(\mathrm{~s}, 3 \mathrm{H}) \mathrm{ppm} ;{ }^{13} \mathrm{C} \mathrm{NMR}\left(100.6 \mathrm{MHz} \mathrm{CDCl}_{3}\right) \delta$ 142.0, 128.5, 126.0, (one aromatic carbon missing), 119.3, 79.8, 69.2, 42.7, 36.3, 33.6, 29.8, 26.1, 22.0, 19.0, 18.4, -3.7, -4.2 ppm; HRMS for $\mathrm{C}_{21} \mathrm{H}_{36} \mathrm{O}_{2} \mathrm{NSi}\left[\mathrm{M}+\mathrm{H}^{+}\right]$: 362.2510, found 362.2513 (0.8 ppm). 26b: $\mathrm{R}_{f}=0.34$ (15:85 EtOAc:Hex); IR (neat) $v_{\max }$ 3435, $2245 \mathrm{~cm}^{-1}$; ${ }^{1} \mathrm{H}$ NMR $\left(500 \mathrm{MHz}, \mathrm{CDCl}_{3}\right) \delta 7.31-7.27(\mathrm{~m}, 2 \mathrm{H}), 7.22-7.14(\mathrm{~m}, 3 \mathrm{H}), 4.51(\mathrm{~d}, J 4.0 \mathrm{~Hz}, 1 \mathrm{H})$, $4.24(\mathrm{~d}, J 4.1 \mathrm{~Hz}, 1 \mathrm{H}), 3.69(\mathrm{dd}, J 5.5,4.0 \mathrm{~Hz}, 1 \mathrm{H}), 2.69-2.51(\mathrm{~m}, 2 \mathrm{H}), 1.90-1.78(\mathrm{~m}, 1 \mathrm{H}), 1.75-1.58(\mathrm{~m}, 2 \mathrm{H})$, $1.54-1.43(\mathrm{~m}, 1 \mathrm{H}), 1.15(\mathrm{~s}, 3 \mathrm{H}), 0.98(\mathrm{~s}, 3 \mathrm{H}), 0.89(\mathrm{~s}, 9 \mathrm{H}), 0.13(\mathrm{~s}, 3 \mathrm{H}), 0.07(\mathrm{~s}, 3 \mathrm{H}) \mathrm{ppm} ;{ }^{13} \mathrm{C} \mathrm{NMR}(100.6 \mathrm{MHz}$, $\left.\mathrm{CDCl}_{3}\right) \delta 141.7,128.6,128.5,126.2,119.0,80.3,69.6,41.7,36.3,33.1,29.4,26.1,21.6,20.7,18.3,-3.8,-4.2$ ppm; HRMS for $\mathrm{C}_{21} \mathrm{H}_{36} \mathrm{O}_{2} \mathrm{NSi}\left[\mathrm{M}+\mathrm{H}^{+}\right]$: 362.2510, found 362.2513 (0.9 ppm).

(士)-(S)-Methyl 4-(benzyloxy)-3-hydroxy-2,2-dimethylbutanoate (S12): To a solution of crude 2-(benzyloxy)acetaldehyde ${ }^{44}\left(1.8 \mathrm{~g}, 12 \mathrm{mmol}, 1.0\right.$ equiv.) in anhydrous $\mathrm{CH}_{2} \mathrm{Cl}_{2}(125 \mathrm{~mL}, 0.10 \mathrm{M})$ at $-78{ }^{\circ} \mathrm{C}, \mathrm{TiCl}_{4}(1.00 \mathrm{M}, 13.8$ $\mathrm{mL}, 13.8 \mathrm{mmol}, 1.15$ equiv.) and commercially available enoxysilane ((1-methoxy-2-methylprop-1-en-1yl)oxy)trimethylsilane) ( $4.9 \mathrm{~mL}, 24 \mathrm{mmol}, 2.0$ equiv.) were added. The resulting solution was stirred until the aldehyde was completely consumed as indicated by TLC (generally $90 \mathrm{~min}$ ). A saturated aqueous solution of 
$\mathrm{NH}_{4} \mathrm{Cl}$ was added and the aqueous layer was extracted with EtOAc $(3 \times)$. The organic layers were combined, washed with brine, dried over $\mathrm{MgSO}_{4}$, filtered and concentrated in vacuo. Purification provided S12 (2.22 g, 73\%) which has been previously reported in the literature. ${ }^{45}$

(士)-(S)-Methyl 4-(benzyloxy)-3-(tert-butyldimethylsilyloxy)-2,2-dimethylbutanoate (S13): To a solution of alcohol S12 (2.2 g, $8.8 \mathrm{mmol}, 1.0$ equiv.) in anhydrous $\mathrm{CH}_{2} \mathrm{Cl}_{2}(87 \mathrm{~mL}, 0.10 \mathrm{M})$ at $0{ }^{\circ} \mathrm{C}, 2,6$-lutidine $(2.0 \mathrm{~mL}, 18$ mmol, 2.0 equiv.) and TBSOTf ( $2.8 \mathrm{~mL}, 12 \mathrm{mmol}, 1.4$ equiv.) were added. The solution was stirred until the alcohol was all consumed (1h $30 \mathrm{~m}$ ). An aqueous solution of $\mathrm{NH}_{4} \mathrm{Cl}$ was added and the aqueous layer was extracted with $\mathrm{Et}_{2} \mathrm{O}(3 \times)$. The organic layers were combined, dried over $\mathrm{MgSO}_{4}$, filtered and concentrated in vacuo. Purification by flash chromatography using 5:95 EtOAc:Hex provided protected ester S13 as a clear oil (2.7 g, 85\% yield): $\mathrm{R}_{f}=0.32$ (10:90 EtOAc:Hex); Molecular formula: $\mathrm{C}_{20} \mathrm{H}_{34} \mathrm{O}_{4} \mathrm{Si}$; MW: 366.57; IR (neat) $v_{\max }$ 2953, 2857, 1736, $1468 \mathrm{~cm}^{-1}$; ${ }^{1} \mathrm{H}$ NMR $\left(500 \mathrm{MHz}, \mathrm{CDCl}_{3}\right) \delta 7.37-7.24(\mathrm{~m}, 5 \mathrm{H}), 4.48(\mathrm{~d}, J 11.8 \mathrm{~Hz}, 1 \mathrm{H}), 4.42(\mathrm{~d}, J$ $11.8 \mathrm{~Hz}, 1 \mathrm{H}$ ), 4.14 (appt, J $5.6 \mathrm{~Hz}, 1 \mathrm{H}$ ), 3.57 (s, 3H), 3.45 (dd, J 9.9, $5.0 \mathrm{~Hz}, 1 \mathrm{H}$ ), 3.38 (dd, J 9.9, $6.2 \mathrm{~Hz}, 1 \mathrm{H}), 1.18$ $(\mathrm{s}, 3 \mathrm{H}), 1.12(\mathrm{~s}, 3 \mathrm{H}), 0.87(\mathrm{~s}, 9 \mathrm{H}), 0.07(\mathrm{~s}, 6 \mathrm{H}) \mathrm{ppm} ;{ }^{13} \mathrm{C} \mathrm{NMR}\left(100.6 \mathrm{MHz}, \mathrm{CDCl}_{3}\right) \delta 177.2,138.2,128.4,127.8$, 127.6, 75.5, 73.4, 72.6, 51.7, 46.7, 26.0, 22.7, 19.4, 18.3, -3.9, -5.0 ppm; HRMS calcd for $\mathrm{C}_{20} \mathrm{H}_{35} \mathrm{O}_{4} \mathrm{Si}\left[\mathrm{M}+\mathrm{H}^{+}\right]$: 367.2299 , found $367.2293(-1.7 \mathrm{ppm})$.

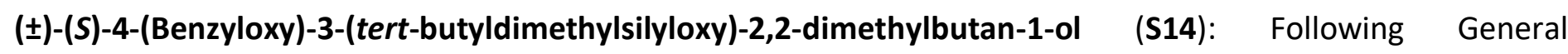
Procedure $A$ and purification by flash chromatography using 25:75 EtOAc:Hex, provided alcohol S14 as a clear oil (2.1 g, 82\% yield): $\mathrm{R}_{f}=0.32$ (25:75 EtOAc:Hex); Molecular formula: $\mathrm{C}_{19} \mathrm{H}_{34} \mathrm{O}_{3} \mathrm{Si}$; $\mathrm{MW}$ : 338.56; IR (neat) $v_{\max }$ 3449, 2956, 2930, 2858, 1471, $1095 \mathrm{~cm}^{-1} ;{ }^{1} \mathrm{H}$ NMR (500 MHz, CDCl $) \delta 7.39-7.26(\mathrm{~m}, 5 \mathrm{H}), 4.51(\mathrm{~s}, 2 \mathrm{H}), 3.67(\mathrm{t}$, J $4.6 \mathrm{~Hz}, 1 \mathrm{H}), 3.63(\mathrm{dd}, J$ 9.9, $4.6 \mathrm{~Hz}, 1 \mathrm{H}), 3.49-3.38(\mathrm{~m}, 3 \mathrm{H}), 3.14(\mathrm{t}, J 6.0 \mathrm{~Hz}, 1 \mathrm{H}), 0.96(\mathrm{~s}, 3 \mathrm{H}), 0.89(\mathrm{~s}, 9 \mathrm{H})$, $0.88(\mathrm{~s}, 3 \mathrm{H}), 0.08(\mathrm{~s}, 3 \mathrm{H}), 0.05(\mathrm{~s}, 3 \mathrm{H}) ;{ }^{13} \mathrm{C} \mathrm{NMR}\left(100.6 \mathrm{MHz}, \mathrm{CDCl}_{3}\right) \delta 137.8,128.5,127.88,127.86,78.5,73.6$, 72.4, 69.9, 39.3, 26.1, 22.5, 22.3, 18.3, -4.0, -4.9 ppm; HRMS calcd for $\mathrm{C}_{19} \mathrm{H}_{35} \mathrm{O}_{3} \mathrm{Si}\left[\mathrm{M}+\mathrm{H}^{+}\right]$: 339.2350, found $339.2347(-0.8 \mathrm{ppm})$.

(士)-(S)-4-(Benzyloxy)-3-(tert-butyldimethylsilyloxy)-2,2-dimethylbutanal (27): Following General Procedure B and purification by flash chromatography using 10:90 EtOAc:Hex provided aldehyde 27 as a clear oil (1.9 g, 91\% yield): $\mathrm{R}_{f}=0.31$ (10:90 EtOAc:Hex); Molecular formula: $\mathrm{C}_{19} \mathrm{H}_{32} \mathrm{O}_{3} \mathrm{Si}$; MW: 336.55 ; IR (neat) $\mathrm{v}_{\max } 1727 \mathrm{~cm}^{-1}$; ${ }^{1} \mathrm{H}$ NMR $\left(500 \mathrm{MHz}, \mathrm{CDCl}_{3}\right) \delta 9.59(\mathrm{~s}, 1 \mathrm{H}), 7.37-7.26(\mathrm{~m}, 5 \mathrm{H}), 4.47(\mathrm{~d}, J 12.2 \mathrm{~Hz}, 1 \mathrm{H}), 4.44(\mathrm{~d}, J 12.0 \mathrm{~Hz}, 1 \mathrm{H}), 3.95$ (t, J $5.3 \mathrm{~Hz}, 1 \mathrm{H}$ ), 3.45 (dd, J 9.6, $5.1 \mathrm{~Hz}, 1 \mathrm{H}), 3.42$ (dd, J 9.6, $5.0 \mathrm{~Hz}, 1 \mathrm{H}), 1.05(\mathrm{~s}, 6 \mathrm{H}), 0.87(\mathrm{~s}, 9 \mathrm{H}), 0.07(\mathrm{~s}, 3 \mathrm{H})$, 0.05 (s, 3H); ${ }^{13} \mathrm{C} \mathrm{NMR}\left(126 \mathrm{MHz}, \mathrm{CDCl}_{3}\right) \delta 205.0,137.9,128.5,127.82,127.79,75.5,73.5,71.8,50.1,26.0,19.1$, 18.3, 17.9, -4.0, -4.9 ppm; HRMS calcd for $\mathrm{C}_{19} \mathrm{H}_{31} \mathrm{O}_{3} \mathrm{Si}\left[\mathrm{M}-\mathrm{H}^{+}\right]$: 335.2048, found 335.2033 (-1.2 ppm).

( \pm )-(2R,4S)-5-(Benzyloxy)-4-(tert-butyldimethylsilyloxy)-2-hydroxy-3,3-imethylpentanenitrile (28a) and ( \pm )(2S,4S)-5-(benzyloxy)-4-(tert-butyldimethylsilyloxy)-2-hydroxy-3,3-dimethylpentanenitrile (28b): Cyanohydrins 28a and 28b were prepared following General Procedure C. ${ }^{1} \mathrm{H}$ NMR spectroscopic analysis of the crude reaction indicated an 11:1 mixture of 2,4-syn and anti diastereomers (Table 1, entry 8 ). Purification by flash chromatography using 15:85 EtOAc:Hex provided 28a and 28b (36.9 mg, 79\% yield) as a clear oil. 28a: $\mathrm{R}_{f}$ $=0.38$ (20:80 EtOAc:Hex); Molecular formula: $\mathrm{C}_{20} \mathrm{H}_{33} \mathrm{NO}_{3} \mathrm{Si} ; \mathrm{MW}: 363.57$; IR (neat) $V_{\max } 3442,2247 \mathrm{~cm}^{-1} ;{ }^{1} \mathrm{H}$ NMR $\left(500 \mathrm{MHz}, \mathrm{CDCl}_{3}\right) \delta 7.40-7.29(\mathrm{~m}, 5 \mathrm{H}), 4.78(\mathrm{~d}, J 6.3 \mathrm{~Hz}, 1 \mathrm{H}), 4.56(\mathrm{~s}, 2 \mathrm{H}), 4.47(\mathrm{~d}, J 5.7 \mathrm{~Hz}, 1 \mathrm{H}), 3.74(\mathrm{dd}$, J 6.0, $3.3 \mathrm{~Hz}, 1 \mathrm{H}$ ), 3.57 (dd, J 10.5, $6.1 \mathrm{~Hz}, 1 \mathrm{H}$ ), 3.50 (dd, J 10.5, 3.3 Hz, 1H), 1.09 (s, 3H), 1.07 (s, $3 \mathrm{H}), 0.88(\mathrm{~s}$, 9H), 0.08 (s, 3H), 0.02 (s, 3H) ppm; $\left.{ }^{13} \mathrm{C} \mathrm{NMR} \mathrm{(100.6} \mathrm{MHz,} \mathrm{CDCl} 3\right) \delta 136.5,128.8,128.5,128.2,119.4,76.2,74.0$, 70.7, 67.7, 42.9, 25.9, 21.5, 20.9, 18.2, -4.1, -4.9 ppm; HRMS for $\mathrm{C}_{20} \mathrm{H}_{34} \mathrm{O}_{3} \mathrm{NSi}\left[\mathrm{M}+\mathrm{H}^{+}\right]$: 364.2302, found 364.2298 (-1.3 ppm). 28b: $R_{f}=0.40$ (20:80 EtOAc:Hex); IR (neat) $v_{\max } 3433,2245 \mathrm{~cm}^{-1} ;{ }^{1} \mathrm{H} \mathrm{NMR}(500 \mathrm{MHz}$, $\left.\mathrm{CDCl}_{3}\right) \delta 7.42-7.28(\mathrm{~m}, 5 \mathrm{H}), 4.55(\mathrm{~d}, J 5.6 \mathrm{~Hz}, 1 \mathrm{H}), 4.53(\mathrm{~d}, J 12.2 \mathrm{~Hz}, 1 \mathrm{H}), 4.50(\mathrm{~d}, J 11.9 \mathrm{~Hz}, 1 \mathrm{H}), 4.35(\mathrm{~d}, J 5.6$ $\mathrm{Hz}, 1 \mathrm{H}$ ), 3.85 (appt, J $4.2 \mathrm{~Hz}, 1 \mathrm{H}$ ), 3.61 (dd, J 10.3, 3.9 Hz, 1H), 3.50 (dd, J 10.3, $4.6 \mathrm{~Hz}, 1 \mathrm{H}$ ), 1.17 (s, $3 \mathrm{H}$ ), 1.03 (s, $3 \mathrm{H}), 0.90$ (s, 9H), 0.13 (s,3H), $0.08(\mathrm{~s}, 3 \mathrm{H}) \mathrm{ppm} ;{ }^{13} \mathrm{C} \mathrm{NMR}\left(100.6 \mathrm{MHz} \mathrm{CDCl}_{3}\right) \delta$ 137.4, 128.6, 128.1, 127.9, 119.0, 
78.4, 73.6, 71.6, 69.8, 41.1, 26.0, 21.4, 21.1, 18.3, -4.1, -5.0 ppm; HRMS calcd for $\mathrm{C}_{20} \mathrm{H}_{34} \mathrm{O}_{3} \mathrm{NSi}\left[\mathrm{M}+\mathrm{H}^{+}\right]$: 364.23025 , found $364.23016(-0.2 \mathrm{ppm})$.

\section{Compounds 4, 29, 30, 31, 32 and 33 from Scheme 7:}

\section{(+)-(5S,6S,7R)-5-[(Benzyloxy)methyl]-7-[bis(ethylthio)methyl]-2,2,3,3,6,9,9,10,10-nonamethyl-6-vinyl-4,8-}

dioxa-3,9-disilaundecane (4): A solution of cyanohydrin 16 (2.2 g, $4.5 \mathrm{mmol}, 1.0$ equiv.) in toluene (9.0 mL, $0.50 \mathrm{M}$ ) was cooled to $0^{\circ} \mathrm{C}$ followed by dropwise addition of DIBAL-H (1.0 M in hexanes, $6.7 \mathrm{~mL}, 6.7 \mathrm{mmol}, 1.4$ equiv.). After stirring 1 hour at $0{ }^{\circ} \mathrm{C}$, saturated aqueous Rochelle salt $(15 \mathrm{~mL})$ was added slowly and the biphasic mixture was warmed to room temperature, diluted with $\mathrm{Et}_{2} \mathrm{O}(10 \mathrm{~mL})$ and stirred vigorously for 2 hours. The aqueous layer was extracted with $\mathrm{Et}_{2} \mathrm{O}(1 \times 15 \mathrm{~mL})$ and the combined organic layers dried over $\mathrm{MgSO}_{4}$, filtered and concentrated in vacuo. The residue was dissolved in EtOAc and $1 \mathrm{~N} \mathrm{HCl}(2: 1,30 \mathrm{~mL})$ was added. The biphasic mixture was stirred vigorously for 1 hour at which point, $\mathrm{Et}_{2} \mathrm{O}$ was added (15 $\left.\mathrm{mL}\right)$, the layers separated, and the organic layer extracted with $\mathrm{Et}_{2} \mathrm{O}(1 \times 15 \mathrm{~mL})$. The combined organic fractions were washed with water followed by brine, dried over $\mathrm{MgSO}_{4}$ and concentrated in vacuo to afford the crude aldehyde $(2.2 \mathrm{~g}$, quantitative yield) which was used immediately for the next step. The aldehyde $(2.2 \mathrm{~g}, 4.5$ mmol, 1.0 equiv.) was dissolved in $\mathrm{CH}_{2} \mathrm{Cl}_{2}(22 \mathrm{~mL}, 0.20 \mathrm{M})$ and the resulting solution was cooled to $-78{ }^{\circ} \mathrm{C}$ followed by addition of ethanethiol (1.0 mL, $14 \mathrm{mmol}, 3.2$ equiv.) and $\mathrm{BF}_{3} \cdot \mathrm{OEt}_{2}(0.72 \mathrm{~mL}, 5.8 \mathrm{mmol}, 1.3$ equiv.). The resulting reaction mixture was stirred 4 hours at $-78^{\circ} \mathrm{C}$ before $\mathrm{Et}_{3} \mathrm{~N}(3.1 \mathrm{~mL}, 23 \mathrm{~mL}, 5.0$ equiv.) was added dropwise with stirring for an additional 20 minutes. Saturated aqueous $\mathrm{NaHCO}_{3}(25 \mathrm{~mL})$ was added slowly and the biphasic mixture warmed to room temperature. After stirring vigorously for 30 minutes, the layers were separated, and the aqueous layer extracted with hexanes $(2 \times 20 \mathrm{~mL})$. The combined organic fractions were washed with $1 \mathrm{~N} \mathrm{NaOH}\left(2 \times 15 \mathrm{~mL}\right.$, to remove excess EtSH), water and brine, dried over $\mathrm{MgSO}_{4}$, filtered and concentrated in vacuo. Purification by flash chromatography (Hexanes/DCM) provided the pure dithioacetal 4 as a slightly yellow oil (1.9 g, 70\% yield): $\mathrm{R}_{f}=0.45$ (Hexanes/ DCM 70:30); $[\alpha]_{\mathrm{D}}^{25}+3.6\left(c=1.6, \mathrm{CH}_{2} \mathrm{Cl}_{2}\right) ; \mathrm{Molecular}$ formula: $\mathrm{C}_{31} \mathrm{H}_{58} \mathrm{O}_{3} \mathrm{~S}_{2} \mathrm{Si}_{2}$; MW: 599.09; IR (neat) $v_{\max }$ 2954, 2927, 2854, $1089 \mathrm{~cm}^{-1} ;{ }^{1} \mathrm{H} \mathrm{NMR}\left(500 \mathrm{MHz} \mathrm{CDCl}_{3}\right) \delta$ $7.35-7.26(\mathrm{~m}, 5 \mathrm{H}), 6.04$ (dd, J 17.6, $10.9 \mathrm{~Hz}, 1 \mathrm{H}), 5.10(\mathrm{dd}, J 10.9,0.9 \mathrm{~Hz}, 1 \mathrm{H}), 4.99(\mathrm{dd}, J 17.6,1.0 \mathrm{~Hz}, 1 \mathrm{H})$, 4.49 (d, J $11.9 \mathrm{~Hz}, 1 \mathrm{H}), 4.45$ (d, J $11.9 \mathrm{~Hz}, 1 \mathrm{H}), 4.12$ (dd, J 7.4, $1.0 \mathrm{~Hz}, 1 \mathrm{H}), 3.99-3.88(\mathrm{~m}, 3 \mathrm{H}), 3.31$ (dd, J 9.5, $7.6 \mathrm{~Hz}, 1 \mathrm{H}), 2.73-2.55(\mathrm{~m}, 2 \mathrm{H}), 2.49$ (q, J $7.4 \mathrm{~Hz}, 2 \mathrm{H}), 1.23(\mathrm{td}, J 7.4,3.5 \mathrm{~Hz}, 6 \mathrm{H}), 1.05(\mathrm{~s}, 3 \mathrm{H}), 0.92(\mathrm{~s}, 9 \mathrm{H}), 0.84$ $(\mathrm{s}, 9 \mathrm{H}), 0.24(\mathrm{~s}, 3 \mathrm{H}), 0.10(\mathrm{~s}, 3 \mathrm{H}), 0.02(\mathrm{~s}, 3 \mathrm{H}), 0.02(\mathrm{~s}, 3 \mathrm{H}) \mathrm{ppm} ;{ }^{13} \mathrm{C} \mathrm{NMR}\left(126 \mathrm{MHz}, \mathrm{CDCl}_{3}\right) \delta 143.9,138.7$, $128.3,127.9,127.4,114.3,82.6,75.3,73.6,73.4,54.7,50.6,27.0,26.61,26.58,26.3,19.0,18.6,15.9,14.6$, 14.4, -2.3, -3.5, -4.4, -4.7 ppm; HRMS calcd for $\mathrm{C}_{31} \mathrm{H}_{58} \mathrm{O}_{3} \mathrm{~S}_{2} \mathrm{Si}_{2} \mathrm{Na}\left[\mathrm{M}+\mathrm{Na}^{+}\right]$: 621.3258; found 621.3240 (-3.0 ppm). (-)-1-((2R,3R,4S)-4-\{(S)-2-(Benzyloxy)-1-[(tert-butyldimethylsilyl)oxy]ethyl\}-3-[(tert-butyldimethylsilyl)oxy]2-(ethylthio)-4-methylhex-5-en-1-yl)-5-methylpyrimidine-2,4(1H,3H)-dione (29): To a solution of dithioacetal 4 (60 mg, $0.10 \mathrm{mmol}, 1.0$ equiv.) in THF $(0.50 \mathrm{~mL}, 0.20 \mathrm{M})$ at $0{ }^{\circ} \mathrm{C}$, silylated thymine $(0.50 \mathrm{M} \mathrm{in} \mathrm{DCE}, 0.40 \mathrm{~mL}$, $0.20 \mathrm{mmol}, 2.0$ equiv.) and iodine (51 $\mathrm{mg}, 0.20 \mathrm{mmol}, 2.0$ equiv.) were added. The resulting dark purple reaction mixture was stirred 16 hours at $0^{\circ} \mathrm{C}$ before addition of saturated aqueous $\mathrm{Na}_{2} \mathrm{~S}_{2} \mathrm{O}_{3}(2 \mathrm{~mL})$ followed by addition of a few drops of $2 \mathrm{~N} \mathrm{NaOH}$. The biphasic mixture was warmed to room temperature and stirred vigorously until a clear homogeneous biphasic mixture was obtained. The aqueous layer was extracted with $\mathrm{Et}_{2} \mathrm{O}(2 \times 2 \mathrm{~mL})$ and the combined organic fractions were washed with brine, dried over $\mathrm{MgSO}_{4}$, filtered and concentrated in vacuo. ${ }^{1} \mathrm{H}$ NMR spectroscopic analysis of the unpurified product indicated the formation of only one diastereomer. Purification by flash chromatography provided thioaminal 29 as a white foam (44 mg, $66 \%$ yield): $\mathrm{R}_{f}=0.54$ (Hexanes/EtOAc, 70:30); $[\alpha]_{\mathrm{D}}^{25}-11$ (c= 1.9, $\mathrm{CH}_{2} \mathrm{Cl}_{2}$ ); Molecular formula: $\mathrm{C}_{34} \mathrm{H}_{58} \mathrm{~N}_{2} \mathrm{O}_{5} \mathrm{SSi}_{2}$; MW: 663.08; IR (neat) $v_{\max } 2927,2855,1676,1089 \mathrm{~cm}^{-1} ;{ }^{1} \mathrm{H} N M R\left(500 \mathrm{MHz}, \mathrm{CDCl}_{3}\right) \delta 8.02(\mathrm{~s}, 1 \mathrm{H}), 7.63$ (d, J 1.2 $\mathrm{Hz}, 1 \mathrm{H}), 7.35-7.28(\mathrm{~m}, 5 \mathrm{H}), 6.01-5.94(\mathrm{~m}, 2 \mathrm{H}), 5.17(\mathrm{dd}, J$ 10.9, $1.3 \mathrm{~Hz}, 1 \mathrm{H}), 5.06(\mathrm{dd}, J 17.7,1.3 \mathrm{~Hz}, 1 \mathrm{H}), 4.45$ (d, J $11.7 \mathrm{~Hz}, 1 \mathrm{H}), 4.41$ (d, J $11.6 \mathrm{~Hz}, 1 \mathrm{H}), 4.23$ (d, J $2.1 \mathrm{~Hz}, 1 \mathrm{H}), 4.13$ (dd, J 7.0, 3.7 Hz, 1H), 3.55 (dd, J 10.0, 3.7 
$\mathrm{Hz}, 1 \mathrm{H}), 3.31(\mathrm{dd}, J$ 10.0, $7.0 \mathrm{~Hz}, 1 \mathrm{H}), 2.41-2.24(\mathrm{~m}, 2 \mathrm{H}), 1.93(\mathrm{~d}, J 1.2 \mathrm{~Hz}, 3 \mathrm{H}), 1.21(\mathrm{t}, J 7.4 \mathrm{~Hz}, 3 \mathrm{H}), 1.11(\mathrm{~s}$, $3 \mathrm{H}), 0.97(\mathrm{~s}, 9 \mathrm{H}), 0.79(\mathrm{~s}, 9 \mathrm{H}), 0.12(\mathrm{~s}, 3 \mathrm{H}), 0.02(\mathrm{~s}, 3 \mathrm{H}), 0.01(\mathrm{~s}, 3 \mathrm{H}),-0.01(\mathrm{~s}, 3 \mathrm{H}) \mathrm{ppm} ;{ }^{13} \mathrm{C} \mathrm{NMR}(126 \mathrm{MHz}$, $\left.\mathrm{CDCl}_{3}\right) \delta 163.6,150.5,141.0,138.3,138.1,128.4,128.0,127.7,116.1,111.2,79.5,73.5,73.4,73.0,64.9,50.5$, 26.6, 26.1, 24.3, 19.3, 18.5, 14.3, 13.9, 12.5, -1.8, -3.53, -3.57, -4.5 ppm; HRMS calcd for $\mathrm{C}_{34} \mathrm{H}_{58} \mathrm{~N}_{2} \mathrm{O}_{5} \mathrm{SSi}_{2} \mathrm{Na}$ $\left[\mathrm{M}+\mathrm{Na}^{+}\right]: 685.3497$; found 685.3487 (-1.4 ppm).

(-)-1-\{(1S,2R,3S)-3-[(S)-2-(Benzyloxy)-1-hydroxyethyl]-2-(tert-butyldimethylsilyl)oxy]-1-(ethylthio)-3-methylpent-4-en-1-yl\}-5-methylpyrimidine-2,4(1H,3H)-dione (30): To a solution of thioaminal 29 (66 mg, $0.10 \mathrm{mmol}$, 1.0 equiv.) in $\mathrm{DCM} / \mathrm{MeOH}(1: 1,5 \mathrm{~mL}, 0.02 \mathrm{M}), \mathrm{TsOH} \cdot \mathrm{H}_{2} \mathrm{O}(95 \mathrm{mg}, 0.50 \mathrm{mmol}, 5.0$ equiv.) was added. The resulting mixture was warmed to $50^{\circ} \mathrm{C}$ and stirring was continued for 16 hours. The reaction mixture was cooled to room temperature and $\mathrm{Et}_{3} \mathrm{~N}(84 \mu \mathrm{L}, 0.60 \mathrm{mmol}, 6.0$ equiv.) was added. After evaporation of the volatiles, the residue was purified by flash chromatography to afford 30 as a white foam (40 $\mathrm{mg}, 73 \%$ yield): $\mathrm{R}_{f}$ $=0.47$ (Hexanes/EtOAc, 60:40); $[\alpha]_{\mathrm{D}}^{25}-51$ (c= 2.0, $\mathrm{CH}_{2} \mathrm{Cl}_{2}$ ); Molecular formula: $\mathrm{C}_{28} \mathrm{H}_{44} \mathrm{~N}_{2} \mathrm{O}_{5} \mathrm{SSi}$; MW: 548.81; IR (neat) $v_{\max } 3427,1678 \mathrm{~cm}^{-1} ;{ }^{1} \mathrm{H}$ NMR $\left(500 \mathrm{MHz}, \mathrm{CDCl}_{3}\right) \delta 8.87(\mathrm{~s}, 1 \mathrm{H}), 7.77(\mathrm{~d}, J 1.2 \mathrm{~Hz}, 1 \mathrm{H}), 7.35-7.31(\mathrm{~m}, 5 \mathrm{H})$, $6.14(\mathrm{~d}, J 2.0 \mathrm{~Hz}, 1 \mathrm{H}), 5.92$ (dd, J 17.6, $10.8 \mathrm{~Hz}, 1 \mathrm{H}), 5.18(\mathrm{dd}, J 10.8,1.2 \mathrm{~Hz}, 1 \mathrm{H}), 5.04(\mathrm{dd}, J 17.7,1.2 \mathrm{~Hz}, 1 \mathrm{H})$, $4.56(\mathrm{~d}, J 12.0 \mathrm{~Hz}, 1 \mathrm{H}), 4.51(\mathrm{~d}, J 12.0 \mathrm{~Hz}, 1 \mathrm{H}), 4.21$ (d, J $1.9 \mathrm{~Hz}, 1 \mathrm{H}), 4.13$ (dd, J 8.4, $2.7 \mathrm{~Hz}, 1 \mathrm{H}$ ), 3.49 (dd, J 10.0, $2.6 \mathrm{~Hz}, 1 \mathrm{H}), 3.33(\mathrm{dd}, J$ 10.0, 8.4 Hz, 1H), $2.48-2.23(\mathrm{~m}, 2 \mathrm{H}), 1.96(\mathrm{~d}, J 1.2 \mathrm{~Hz}, 3 \mathrm{H}), 1.25(\mathrm{t}, J 7.4 \mathrm{~Hz}, 3 \mathrm{H}), 1.12(\mathrm{~s}$, $3 \mathrm{H}), 0.94(\mathrm{~s}, 9 \mathrm{H}), 0.05(\mathrm{~s}, 3 \mathrm{H}), 0.03(\mathrm{~s}, 3 \mathrm{H}) \mathrm{ppm}$, OH signal missing possibly due to exchange in $\mathrm{CDCl}_{3} ;{ }^{13} \mathrm{C} \mathrm{NMR}$ $\left(126 \mathrm{MHz}_{1} \mathrm{CDCl}_{3}\right) \delta 163.6,151.5,140.5,138.3,138.1,128.5,127.9,127.7,117.0,111.4,79.3,73.8,73.4,72.5$, $64.4,49.1,26.5,24.3,19.1,14.5,14.4,12.6,-1.4,-3.1$ ppm; HRMS calcd for $\mathrm{C}_{28} \mathrm{H}_{44} \mathrm{~N}_{2} \mathrm{O}_{5} \mathrm{SSiNa}\left[\mathrm{M}+\mathrm{Na}^{+}\right]$: 571.2632; found 571.2669 (+6.5 ppm).

(-)-1-\{(1S,2R,3S)-3-[(S)-2-(Benzyloxy)-1-hydroxyethyl]-2-[(tert-butyldimethylsilyl)oxy]-1-(ethylthio)-3methylpent-4-en-1-yl\}pyrimidine-2,4(1H,3H)-dione (31): To a $0{ }^{\circ} \mathrm{C}$ solution of dithioacetal $4(72 \mathrm{mg}, 0.12$ mmol, 1.0 equiv.) in THF ( $0.50 \mathrm{~mL}, 0.24 \mathrm{M})$, silylated uracil (0.90 M in DCE, $0.25 \mathrm{~mL}, 0.24 \mathrm{mmol}, 2.0$ equiv.) and iodine (61 $\mathrm{mg}, 0.24 \mathrm{mmol}, 2.0$ equiv.) were added. The resulting dark purple reaction mixture was stirred 16 hours at $0{ }^{\circ} \mathrm{C}$ before addition of saturated aqueous $\mathrm{Na}_{2} \mathrm{~S}_{2} \mathrm{O}_{3}(2 \mathrm{~mL})$ followed by addition of a few drops of $2 \mathrm{~N}$ $\mathrm{NaOH}$. The biphasic mixture was warmed to room temperature and stirred vigorously until a clear homogeneous biphasic mixture was obtained. The aqueous layer was extracted with $\mathrm{Et}_{2} \mathrm{O}(2 \times 2 \mathrm{~mL})$ and the combined organic fractions were washed with brine, dried over $\mathrm{MgSO}_{4}$, filtered and concentrated in vacuo to afford the crude thioaminal which was used directly in the next step. ${ }^{1} \mathrm{H}$ NMR spectroscopic analysis of the unpurified product indicated the formation of only one diastereomer. To a solution of the crude thioaminal in $\mathrm{CH}_{2} \mathrm{Cl}_{2} / \mathrm{MeOH}(1: 1,6.0 \mathrm{~mL}, 0.020 \mathrm{M}), \mathrm{TsOH} \cdot \mathrm{H}_{2} \mathrm{O}(114 \mathrm{mg}, 0.60 \mathrm{mmol}, 5.0$ equiv.) was added. The resulting mixture was warmed to $50^{\circ} \mathrm{C}$ and stirred for 16 hours. The reaction mixture was cooled to room temperature and $\mathrm{Et}_{3} \mathrm{~N}$ (0.10 mL, $0.72 \mathrm{mmol}, 6.0$ equiv.) was added. After evaporation of the volatiles, the residue was purified by flash chromatography to afford 31 as a white foam (51 mg, 79\% yield over two steps): $\mathrm{R}_{f}=0.24$ (Hexanes/EtOAc, 60:40); $[\alpha]_{D}^{25}-48\left(c=1.1, \mathrm{CH}_{2} \mathrm{Cl}_{2}\right)$; Molecular formula: $\mathrm{C}_{27} \mathrm{H}_{42} \mathrm{~N}_{2} \mathrm{O}_{5} \mathrm{SSi} ; \mathrm{MW}$ : 534.79 ; IR (neat) $v_{\max } 3427,1684 \mathrm{~cm}^{-1} ;{ }^{1} \mathrm{H}$ NMR $\left(500 \mathrm{MHz}, \mathrm{CDCl}_{3}\right) \delta 8.87(\mathrm{~s}, 1 \mathrm{H}), 7.97(\mathrm{~d}, J 8.2 \mathrm{~Hz}, 1 \mathrm{H}), 7.35-7.27(\mathrm{~m}, 5 \mathrm{H}), 6.13$ (d, J $1.6 \mathrm{~Hz}, 1 \mathrm{H}$ ), 5.93 (dd, J 17.6, $10.9 \mathrm{~Hz}, 1 \mathrm{H}$ ), 5.79 (appdd, J 8.1, 2.2 Hz, 1H), 5.19 (d, J $10.9 \mathrm{~Hz}, 1 \mathrm{H}), 5.05$ (d, J $17.7 \mathrm{~Hz}, 1 \mathrm{H}), 4.56(\mathrm{~d}, J 11.9 \mathrm{~Hz}, 1 \mathrm{H}), 4.50(\mathrm{~d}, J 11.9 \mathrm{~Hz}, 1 \mathrm{H}), 4.21(\mathrm{~d}, J 1.9 \mathrm{~Hz}, 1 \mathrm{H}), 4.13-4.09(\mathrm{~m}, 1 \mathrm{H}), 3.50(\mathrm{dd}, J$ 9.9, $2.5 \mathrm{~Hz}, 1 \mathrm{H}), 3.38(\mathrm{~s}, 1 \mathrm{H}), 3.33(\mathrm{dd}, J 9.8,8.6 \mathrm{~Hz}, 1 \mathrm{H}), 2.44-2.29(\mathrm{~m}, 2 \mathrm{H}), 1.24(\mathrm{t}, J 7.4 \mathrm{~Hz}, 3 \mathrm{H}), 1.12(\mathrm{~s}, 3 \mathrm{H})$, $0.92(\mathrm{~s}, 9 \mathrm{H}), 0.04(\mathrm{~s}, 3 \mathrm{H}), 0.02(\mathrm{~s}, 3 \mathrm{H}) \mathrm{ppm} ;{ }^{13} \mathrm{C} \mathrm{NMR}\left(126 \mathrm{MHz}, \mathrm{CDCl}_{3}\right) \delta 162.9,151.4,142.5,140.4,138.2$, 128.5, 127.9, 127.8, 117.0, 102.8, 79.1, 73.6, 73.4, 72.3, 65.0, 49.1, 26.5, 24.4, 19.1, 14.42, 14.39, -1.5, -3.0 ppm; HRMS calcd for: $\mathrm{C}_{27} \mathrm{H}_{42} \mathrm{~N}_{2} \mathrm{O}_{5} \mathrm{SSiNa}\left[\mathrm{M}+\mathrm{Na}^{+}\right]$: 557.2476 ; found 557.2470 (-1.1 ppm).

$(-)-1-\{(2 R, 3 R, 4 S, 5 S)-5-[(B e n z y l o x y)$ methyl]-3-[(tert-butyldimethylsilyl)oxy)]-methyl-4-vinyltetrahydrofuran2-yl\}-5-methylpyrimidine-2,4(1H,3H)-dione (32): To a stirred solution of 30 (0.21 g, $0.38 \mathrm{mmol}, 1.0$ equiv.) in 
THF (3.8 mL, $0.10 \mathrm{M}), \mathrm{Me}_{2} \mathrm{~S}(\mathrm{SMe}) \mathrm{BF}_{4}(90 \mathrm{mg}, 0.46 \mathrm{mmol}, 1.2$ equiv.) was added and the resulting mixture was stirred 2 hours at room temperature. The reaction mixture was then diluted with $\mathrm{Et}_{2} \mathrm{O}(15 \mathrm{~mL})$ followed by addition of saturated aqueous $\mathrm{NaHCO}_{3}$. The aqueous phase was extracted with $\mathrm{Et}_{2} \mathrm{O}(1 \times 15 \mathrm{~mL})$ and the combined organic fractions were washed with brine, dried over $\mathrm{MgSO}_{4}$, filtered and concentrated in vacuo. Purification by flash chromatography on silica gel provided 32 as a white foam $\left(0.42 \mathrm{~g}, 71 \%\right.$ yield): $\mathrm{R}_{f}=0.49$ (Hexanes/EtOAc, 60:40); $[\alpha]_{\mathrm{D}}^{25}-12\left(c=0.23, \mathrm{CH}_{2} \mathrm{Cl}_{2}\right.$ ); Molecular formula: $\mathrm{C}_{26} \mathrm{H}_{38} \mathrm{~N}_{2} \mathrm{O}_{5} \mathrm{Si}$; $\mathrm{MW}$ : 486.68; IR (neat) $v_{\max } 3182,3062,2928,1691,1264 \mathrm{~cm}^{-1} ;{ }^{1} \mathrm{H}$ NMR $\left(500 \mathrm{MHz}, \mathrm{CDCl}_{3}\right) 7.92(\mathrm{~s}, 1 \mathrm{H}), 7.76(\mathrm{~d}, J 1.1 \mathrm{~Hz}, 1 \mathrm{H}), 7.39-$ $7.30(\mathrm{~m}, 5 \mathrm{H}), 6.14(\mathrm{dd}, J$ 17.5, $10.9 \mathrm{~Hz}, 1 \mathrm{H}), 6.09(\mathrm{~d}, J 7.4 \mathrm{~Hz}, 1 \mathrm{H}), 5.29(\mathrm{~d}, J 10.9 \mathrm{~Hz}, 1 \mathrm{H}), 5.25(\mathrm{~d}, J 17.5 \mathrm{~Hz}, 1 \mathrm{H})$, $4.64(\mathrm{~d}, J 11.3 \mathrm{~Hz}, 1 \mathrm{H}), 4.54(\mathrm{~d}, J 11.3 \mathrm{~Hz}, 1 \mathrm{H}), 4.37(\mathrm{~d}, J 7.4 \mathrm{~Hz}, 1 \mathrm{H}), 3.89$ (t, J $2.0 \mathrm{~Hz}, 1 \mathrm{H}), 3.77$ (dd, J $10.7,2.6$ $\mathrm{Hz}, 1 \mathrm{H}), 3.55$ (dd, J 10.7, $1.5 \mathrm{~Hz}, 1 \mathrm{H}), 1.38$ (d, J $0.9 \mathrm{~Hz}, 3 \mathrm{H}), 1.33(\mathrm{~s}, 3 \mathrm{H}), 0.81(\mathrm{~s}, 9 \mathrm{H}),-0.04(\mathrm{~s}, 3 \mathrm{H}),-0.16(\mathrm{~s}, 3 \mathrm{H})$ ppm; ${ }^{13} \mathrm{C} \mathrm{NMR}\left(126 \mathrm{MHz}, \mathrm{CDCl}_{3}\right) \delta 163.4,150.8,139.8,137.4,136.6,128.9,128.4,127.6,117.5,111.4,87.2$, 86.7, 79.8, 73.6, 71.4, 49.6, 25.7, 18.0, 17.8, 11.7, -4.1, -4.8 ppm; HRMS calcd for: $\mathrm{C}_{26} \mathrm{H}_{38} \mathrm{~N}_{2} \mathrm{O}_{5} \mathrm{SiNa}\left[\mathrm{M}+\mathrm{Na}^{+}\right]$: 509.2442 ; found 509.2447 (+0.9 ppm).

(-)-1-\{(2R,3R,4S,5S)-5-[(Benzyloxy)methyl]-3-[(tert-butyldimethylsilyl)oxy]-4-methyl-4-vinyltetrahydrofuran2-yl\}pyrimidine-2,4(1H,3H)-dione (33): To a stirred solution of 31 (0.12 g, $0.22 \mathrm{mmol}, 1.0$ equiv.) in THF (2.2 $\mathrm{mL}, 0.10 \mathrm{M}$ ), $\mathrm{Me}_{2} \mathrm{~S}(\mathrm{SMe}) \mathrm{BF}_{4}(55 \mathrm{mg}, 0.28 \mathrm{mmol}, 1.3$ equiv.) was added and the resulting mixture was stirred 2 hours at room temperature. The reaction mixture was diluted with $\mathrm{Et}_{2} \mathrm{O}(5 \mathrm{~mL})$ followed by addition of saturated aqueous $\mathrm{NaHCO}_{3}$. The aqueous phase was extracted with $\mathrm{Et}_{2} \mathrm{O}(1 \times 15 \mathrm{~mL})$ and the combined organic fractions were washed with brine, dried over $\mathrm{MgSO}_{4}$, filtered and concentrated in vacuo. Purification by flash chromatography on silica gel provided 33 as a white foam (81 $\mathrm{mg}, 80 \%$ yield): $\mathrm{R}_{f}=0.49$ (Hexanes/EtOAc, 60:40); $[\alpha]_{D}^{25}-1.6\left(c=0.19, \mathrm{CH}_{2} \mathrm{Cl}_{2}\right.$ ); Molecular formula: $\mathrm{C}_{25} \mathrm{H}_{36} \mathrm{~N}_{2} \mathrm{O}$ 列; MW: 472.66; IR (neat) $V_{\max } 3183,3064,2928,1686,1265,1059,837 \mathrm{~cm}^{-1} ;{ }^{1} \mathrm{H} \mathrm{NMR}\left(500 \mathrm{MHz}, \mathrm{CDCl}_{3}\right) \delta 7.93(\mathrm{~d}, J=8.2 \mathrm{~Hz}, 1 \mathrm{H}), 7.88(\mathrm{~s}$, $1 \mathrm{H}), 7.44-7.34(\mathrm{~m}, 5 \mathrm{H}), 6.08(\mathrm{dd}, J=17.5,10.8 \mathrm{~Hz}, 1 \mathrm{H}), 6.06(\mathrm{~d}, J=7.3 \mathrm{~Hz}, 1 \mathrm{H}), 5.30(\mathrm{dd}, J=10.9,0.7 \mathrm{~Hz}, 1 \mathrm{H})$, $5.24(\mathrm{dd}, J=17.7,0.8 \mathrm{~Hz}, 1 \mathrm{H}), 5.21(\mathrm{dd}, J=8.2,2.5 \mathrm{~Hz}, 1 \mathrm{H}), 4.61(\mathrm{~d}, J=10.4 \mathrm{~Hz}, 1 \mathrm{H}), 4.43(\mathrm{~d}, J=10.5 \mathrm{~Hz}, 1 \mathrm{H})$, $4.26(\mathrm{~d}, J=7.3 \mathrm{~Hz}, 1 \mathrm{H}), 3.88(\mathrm{dd}, J=2.2,1.7 \mathrm{~Hz}, 1 \mathrm{H}), 3.75(\mathrm{dd}, J=10.6,2.6 \mathrm{~Hz}, 1 \mathrm{H}), 3.57(\mathrm{dd}, J=10.6,1.4 \mathrm{~Hz}$, $1 \mathrm{H}), 1.31(\mathrm{~s}, 3 \mathrm{H}), 0.79(\mathrm{~s}, 9 \mathrm{H}),-0.10(\mathrm{~s}, 3 \mathrm{H}),-0.22(\mathrm{~s}, 3 \mathrm{H}) \mathrm{ppm} ;{ }^{13} \mathrm{C} \mathrm{NMR}\left(126 \mathrm{MHz} \mathrm{CDCl}_{3}\right) \delta 162.7,150.7,141.2$, 139.7, 137.2, 129.0, 128.7, 128.3, 117.6, 102.5, 87.4, 86.9, 79.9, 73.9, 71.3, 49.7, 25.7, 17.9, 17.7, -4.2, -4.9 ppm; HRMS calcd for: $\mathrm{C}_{25} \mathrm{H}_{36} \mathrm{~N}_{2} \mathrm{O}_{5} \mathrm{SiNa}\left[\mathrm{M}+\mathrm{Na}^{+}\right]$: 495.2286; found 495.2291 (+1.0 ppm).

\section{Compounds 34, 35, 36 and 37 from Scheme 8:}

(+)-1-\{(2R,3R,4R,5S)-5-[(Benzyloxy)methyl]-3-hydroxy-4-(hydroxymethyl)-4-methyltetrahydrofuran-2-yl\}-5methylpyrimidine-2,4(1H,3H)-dione (34): To a solution of alkene $32(0.11 \mathrm{~g}, 0.22 \mathrm{mmol}, 1.0$ equiv.) in acetone $/ \mathrm{H}_{2} \mathrm{O}(2: 1,2.2 \mathrm{~mL}, 0.10 \mathrm{M})$, NMO (0.10 g, $0.86 \mathrm{mmol}, 4.0$ equiv.) and $\mathrm{OsO}_{4}\left(4 \%\right.$ in $\mathrm{H}_{2} \mathrm{O}, 70 \mu \mathrm{L}, 0.050$ equiv.) were added. The resulting mixture was warmed to $50^{\circ} \mathrm{C}$ and stirring was continued for 16 hours. After cooling to room temperature, the reaction mixture was diluted with EtOAc $(3 \mathrm{~mL})$ and water $(2 \mathrm{~mL})$. The aqueous layer was extracted with EtOAc $(2 \times 3 \mathrm{~mL})$ and the combined organic fractions were washed with brine, dried over $\mathrm{MgSO}_{4}$, filtered and concentrated in vacuo to provide the crude diol as a single diastereoisomer (unassigned stereochemistry) which was used directly in the next step. The crude diol ( $0.22 \mathrm{mmol}, 1.0$ equiv.) was dissolved in dioxane $/ \mathrm{H}_{2} \mathrm{O}(9: 1,2.2 \mathrm{~mL}, 0.10 \mathrm{M})$ and the resulting solution was cooled to $0{ }^{\circ} \mathrm{C}$ followed by addition of $\mathrm{NaIO}_{4}(94 \mathrm{mg}, 0.44 \mathrm{mmol}, 2.0$ equiv.). The mixture was warmed to room temperature and stirring was continued for 3 hours. The reaction mixture was diluted with EtOAc $(2 \mathrm{~mL})$ and slowly poured into stirring cold saturated aqueous $\mathrm{Na}_{2} \mathrm{~S}_{2} \mathrm{O}_{3}$. After stirring 15 minutes, the aqueous phase was extracted with EtOAc ( $2 \times 3$ $\mathrm{mL}$ ) and the combined organic fractions were washed with brine, dried over $\mathrm{MgSO}_{4}$, filtered and concentrated in vacuo to provide the crude aldehyde as a white solid. The crude aldehyde $(0.22 \mathrm{mmol}, 1.0$ equiv. $)$ was dissolved in THF $(2.2 \mathrm{~mL}, 0.10 \mathrm{M})$ and the resulting solution cooled to $-40{ }^{\circ} \mathrm{C}$. $\mathrm{LiBH}_{4}(2.0 \mathrm{M} \mathrm{in} \mathrm{THF,} 0.14 \mathrm{~mL}$, 
0.29 mmol, 1.3 equiv.) was added dropwise and stirring was continued for 2 hours. The reaction was quenched with slow addition of $0.05 \mathrm{~N} \mathrm{HCl}(2 \mathrm{~mL})$ and warmed to room temperature. After stirring vigorously for 30 minutes, EtOAc $(3 \mathrm{~mL})$ was added and the aqueous phase extracted with EtOAc $(2 \times 3 \mathrm{~mL})$. The combined organic fractions were washed with brine, dried over $\mathrm{MgSO}_{4}$, filtered and concentrated in vacuo to provide the crude alcohol. The crude alcohol $(0.22 \mathrm{mmol}, 1.0$ equiv.) was dissolved in THF (1.1 mL, $0.20 \mathrm{M})$ and the resulting solution was cooled to $0{ }^{\circ} \mathrm{C}$. TBAF $(1.0 \mathrm{M}$ in THF, $0.33 \mathrm{~mL}, 0.33 \mathrm{mmol}, 1.5$ equiv.) was added and stirring at $0{ }^{\circ} \mathrm{C}$ was continued for 16 hours. The reaction mixture was diluted with EtOAc ( $\left.3 \mathrm{~mL}\right)$ followed by addition of saturated aqueous $\mathrm{NH}_{4} \mathrm{Cl}$. The aqueous layer was extracted with EtOAc $(2 \times 3 \mathrm{~mL})$ and the combined organic fractions were washed with brine, dried over $\mathrm{MgSO}_{4}$, filtered and concentrated in vacuo to provide the crude diol. Purification by flash chromatography on silica gel provided the pure $\mathrm{C}^{\prime}$-protected nucleoside 34 as a white foam (49 mg, 59\% yield over 4 steps): $\mathrm{R}_{f}=0.64\left(\mathrm{CH}_{2} \mathrm{Cl}_{2} / \mathrm{MeOH}, 90: 10\right) ;[\alpha]_{\mathrm{D}}^{25}+1.9\left(c=0.66, \mathrm{CH}_{2} \mathrm{Cl}_{2} / \mathrm{MeOH}\right.$ 10:1); Molecular formula: $\mathrm{C}_{19} \mathrm{H}_{24} \mathrm{~N}_{2} \mathrm{O}_{6}$; $\mathrm{MW}: 376.41$; IR (neat) $v_{\max } 3412,1685 \mathrm{~cm}^{-1} ;{ }^{1} \mathrm{H} \mathrm{NMR}(500 \mathrm{MHz}, \mathrm{CDCl} 3)$ $\delta 10.97(\mathrm{~s}, 1 \mathrm{H}), 7.60(\mathrm{~s}, 1 \mathrm{H}), 7.38-7.28(\mathrm{~m}, 5 \mathrm{H}), 5.76(\mathrm{~d}, J 4.4 \mathrm{~Hz}, 1 \mathrm{H}), 5.01(\mathrm{~s}, 1 \mathrm{H}), 4.62(\mathrm{~d}, J 11.6 \mathrm{~Hz}, 1 \mathrm{H}), 4.58$ (d, J $11.6 \mathrm{~Hz}, 1 \mathrm{H}), 4.42(\mathrm{~s}, 1 \mathrm{H}), 4.10(\mathrm{t}, J 3.6 \mathrm{~Hz}, 1 \mathrm{H}), 3.82(\mathrm{dd}, J 10.9,3.4 \mathrm{~Hz}, 1 \mathrm{H}), 3.77(\mathrm{dd}, J 10.9,3.9 \mathrm{~Hz}, 1 \mathrm{H})$, $3.63(\mathrm{~s}, 2 \mathrm{H}), 3.57(\mathrm{~s}, 1 \mathrm{H}), 1.52(\mathrm{~s}, 3 \mathrm{H}), 1.19(\mathrm{~s}, 3 \mathrm{H}) \mathrm{ppm} ;{ }^{13} \mathrm{C} \mathrm{NMR}\left(126 \mathrm{MHz}, \mathrm{CDCl}_{3}\right) \delta 164.5,152.3,137.4,136.9$, 128.8, 128.2, 127.9, 109.8, 91.8, 86.1, 78.4, 73.9, 69.2, 66.1, 48.0, 16.6, 12.3 ppm; HRMS calcd for $\mathrm{C}_{19} \mathrm{H}_{24} \mathrm{~N}_{2} \mathrm{O}_{6} \mathrm{Na}\left[\mathrm{M}+\mathrm{Na}^{+}\right]: 399.1527$; found 399.1527 (0.0 ppm).

(+)-1-\{(2R,3R,4R,5S)-5-[(Benzyloxy)methyl]-3-hydroxy-4-(hydroxymethyl)-4-methyltetrahydrofuran-2-yl\}pyrimidine-2,4(1H,3H)-dione (35): To a solution of alkene 33 (47 mg, $0.10 \mathrm{mmol}, 1.0$ equiv.) in acetone/ $\mathrm{H}_{2} \mathrm{O}$ $(2: 1,1.0 \mathrm{~mL}, 0.10 \mathrm{M}), \mathrm{NMO}\left(47 \mathrm{mg}, 0.40 \mathrm{mmol}, 4.0\right.$ equiv.) and $\mathrm{OsO}_{4}\left(4 \%\right.$ in $\mathrm{H}_{2} \mathrm{O}, 33 \mu \mathrm{L}, 0.050$ equiv.) were added. The resulting mixture was warmed to $50{ }^{\circ} \mathrm{C}$ and stirring was continued for 16 hours. After cooling to room temperature, the reaction mixture was diluted with EtOAc $(2 \mathrm{~mL})$ and water $(1 \mathrm{~mL})$. The aqueous layer was extracted with EtOAc $(2 \times 2 \mathrm{~mL})$ and the combined organic fractions were washed with brine, dried over $\mathrm{MgSO}_{4}$, filtered and concentrated in vacuo to provide the crude diol as a single diastereoisomer (unassigned stereochemistry) which was used directly in the next step. The crude diol was dissolved in dioxane/ $\mathrm{H}_{2} \mathrm{O}(9: 1$, $1.0 \mathrm{~mL}, 0.10 \mathrm{M})$ and the resulting solution cooled to $0{ }^{\circ} \mathrm{C}$ followed by addition of $\mathrm{NaIO}_{4}(43 \mathrm{mg}, 0.20 \mathrm{mmol}, 2.0$ equiv.). The mixture was warmed to room temperature and stirring was continued for 3 hours. The reaction mixture was diluted with EtOAc $(2 \mathrm{~mL})$ and slowly poured into stirring cold saturated aqueous $\mathrm{Na}_{2} \mathrm{~S}_{2} \mathrm{O}_{3}$. After stirring 15 minutes, the aqueous phase was extracted with EtOAc $(2 \times 3 \mathrm{~mL})$. The combined organic fractions were washed with brine, dried over $\mathrm{MgSO}_{4}$, filtered and concentrated in vacuo to provide the crude aldehyde as a white solid. The crude aldehyde was dissolved in THF $(1.0 \mathrm{~mL}, 0.10 \mathrm{M})$ and the resulting solution cooled to $-40{ }^{\circ} \mathrm{C}$. $\mathrm{LiBH}_{4}(2.0 \mathrm{M}$ in THF, $50 \mu \mathrm{L}, 0.10 \mathrm{mmol}, 1.0$ equiv.) was added dropwise and stirring was continued for 2 hours. The reaction was quenched with slow addition of $0.05 \mathrm{~N} \mathrm{HCl}(2.5 \mathrm{~mL})$ and warmed to room temperature. After stirring vigorously for 30 minutes, EtOAc $(3 \mathrm{~mL})$ was added, and the aqueous phase was extracted with EtOAc $(2 \times 3 \mathrm{~mL})$. The combined organic fractions were washed with brine, dried over $\mathrm{MgSO}_{4}$, filtered and concentrated in vacuo to provide the crude alcohol. The crude alcohol was dissolved in THF $(0.50$ $\mathrm{mL}, 0.20 \mathrm{M})$ and the resulting solution cooled to $0^{\circ} \mathrm{C}$. TBAF $(1.0 \mathrm{M}$ in THF, $0.15 \mathrm{~mL}, 0.15 \mathrm{mmol}, 1.5$ equiv.) was added and stirring at $0{ }^{\circ} \mathrm{C}$ continued for 16 hours. The reaction mixture was diluted with EtOAc ( $\left.3 \mathrm{~mL}\right)$ followed by addition of saturated aqueous $\mathrm{NH}_{4} \mathrm{Cl}$. The aqueous layer was extracted with EtOAc $(2 \times 3 \mathrm{~mL})$ and the combined organic fractions were washed with brine, dried over $\mathrm{MgSO}_{4}$, filtered and concentrated in vacuo to provide the crude diol. Purification by flash chromatography on silica gel provided the pure $\mathrm{C}^{\prime}$ ' protected nucleoside 35 as a white foam (21.7 mg, 59\% yield over 4 steps): $\mathrm{R}_{f}=0.61\left(\mathrm{CH}_{2} \mathrm{Cl}_{2} / \mathrm{MeOH}, 90: 10\right) ;[\alpha]_{\mathrm{D}}^{25}+5.0(c=$ 0.64, $\mathrm{CH}_{2} \mathrm{Cl}_{2} / \mathrm{MeOH} 10: 1$ ); Molecular formula: $\mathrm{C}_{18} \mathrm{H}_{22} \mathrm{~N}_{2} \mathrm{O}_{6}$; MW: 362.38; IR (neat) $v_{\max } 3392,1680 \mathrm{~cm}^{-1} ;{ }^{1} \mathrm{H} \mathrm{NMR}$ $\left(500 \mathrm{MHz}, \mathrm{CD}_{3} \mathrm{OD}\right) \delta 7.99(\mathrm{~d}, J 8.1 \mathrm{~Hz}, 1 \mathrm{H}), 7.42-7.30(\mathrm{~m}, 5 \mathrm{H}), 5.93(\mathrm{~d}, J 6.9 \mathrm{~Hz}, 1 \mathrm{H}), 5.31(\mathrm{~d}, J 8.1 \mathrm{~Hz}, 1 \mathrm{H}), 4.62$ 
(d, J $10.8 \mathrm{~Hz}, 1 \mathrm{H}$ ), 4.49 (d, J $10.8 \mathrm{~Hz}, 1 \mathrm{H}), 4.08$ (d, J $6.9 \mathrm{~Hz}, 1 \mathrm{H}), 3.99$ (t, J $2.9 \mathrm{~Hz}, 1 \mathrm{H}), 3.89-3.79(\mathrm{~m}, 3 \mathrm{H}), 3.59$ $(\mathrm{d}, J 10.9 \mathrm{~Hz}, 1 \mathrm{H}), 1.24(\mathrm{~s}, 3 \mathrm{H}) \mathrm{ppm}, \mathrm{OH}$ and $\mathrm{NH}$ signals missing possibly due to exchange in $\mathrm{CD}_{3} \mathrm{OD} ;{ }^{13} \mathrm{C} \mathrm{NMR}$ (126 MHz, CD $\left.{ }_{3} \mathrm{OD}\right) \delta 166.1,152.9,142.9,139.1,129.7,129.4,129.2,102.6,89.6,87.4,77.8,74.7,71.5,66.1$, 49.0, 17.5 ppm; HRMS calcd for $\mathrm{C}_{18} \mathrm{H}_{22} \mathrm{~N}_{2} \mathrm{O}_{6} \mathrm{Na}\left[\mathrm{M}+\mathrm{Na}^{+}\right]$: 385.1370 ; found 385.1369 (-0.3 ppm).

(+)-1-[(2R,3R,4R,5S)-3-Hydroxy-4,5-bis(hydroxymethyl)-4-(methyltetrahydrofuran-2-yl)]-5-methylpyrimidine2,4(1H,3H)-dione (36): To a $-40^{\circ} \mathrm{C}$ solution of benzyl ether 34 (47 mg, $0.13 \mathrm{mmol}, 1.0$ equiv.) in $\mathrm{CH}_{2} \mathrm{Cl}_{2}(3.1 \mathrm{~mL}$, $0.025 \mathrm{M}), \mathrm{BCl}_{3}\left(1.0 \mathrm{M}\right.$ in $\mathrm{CH}_{2} \mathrm{Cl}_{2}, 0.63 \mathrm{~mL}, 0.63 \mathrm{mmol}, 5.0$ equiv.) was added dropwise. The reaction mixture was stirred for 3 hours, at which point, methanol $(0.10 \mathrm{~mL})$ was added. With warming to room temperature, stirring was continued for 30 minutes and the volatiles removed under reduced pressure. ${ }^{1} \mathrm{H}$ NMR analysis of the crude reaction mixture showed an 8:1 mixture of the product and starting material. Purification by flash chromatography (Biotage, C18 reverse phase, Water/MeOH) provided nucleoside $\mathbf{3 6}$ as a white foam (16.4 $\mathrm{mg}, 46 \%$ yield): $[\alpha]_{\mathrm{D}}^{25}+3.2$ ( $c=1.3, \mathrm{MeOH}$ ); Molecular formula: $\mathrm{C}_{12} \mathrm{H}_{18} \mathrm{~N}_{2} \mathrm{O}_{6}$; MW: 286.28; IR (neat) $\mathrm{v}_{\max } 2322$, $1667 \mathrm{~cm}^{-1} ;{ }^{1} \mathrm{H}$ NMR $\left(500 \mathrm{MHz}, \mathrm{CD}_{3} \mathrm{OD}\right) \delta 7.99(\mathrm{~d}, J 1.1 \mathrm{~Hz}, 1 \mathrm{H}), 5.90(\mathrm{~d}, J 6.9 \mathrm{~Hz}, 1 \mathrm{H}), 4.23(\mathrm{~d}, J 6.8 \mathrm{~Hz}, 1 \mathrm{H}), 3.88$ - $3.78(\mathrm{~m}, 3 \mathrm{H}), 3.71(\mathrm{~d}, J 11.2 \mathrm{~Hz}, 1 \mathrm{H}), 3.57(\mathrm{~d}, J 11.2 \mathrm{~Hz}, 1 \mathrm{H}), 1.89(\mathrm{~d}, J 0.9 \mathrm{~Hz}, 3 \mathrm{H}), 1.15(\mathrm{~s}, 3 \mathrm{H}) \mathrm{ppm}, \mathrm{OH}$ and $\mathrm{NH}$ signals missing possibly due to exchange in $\mathrm{CD}_{3} \mathrm{OD} ;{ }^{13} \mathrm{C} N \mathrm{NMR}\left(126 \mathrm{MHz}, \mathrm{CD}_{3} \mathrm{OD}\right) \delta 166.4,153.1,138.7$, 111.6, 89.6, 88.5, 77.0, 65.5, 62.7, 48.6, 17.8, 12.5 ppm; HRMS calcd for $\mathrm{C}_{12} \mathrm{H}_{18} \mathrm{~N}_{2} \mathrm{O}_{6} \mathrm{Na}\left[\mathrm{M}+\mathrm{Na}^{+}\right]: 309.1057$; found $309.1065(+2.6 \mathrm{ppm})$. See supporting information for proof of structure.

(+)-1-[(2R,3R,4R,5S)-3-Hydroxy-4,5-bis(hydroxymethyl)-4-methyltetrahydrofuran-2-yl]pyrimidine-2,4(1H,3H)dione (37): To a $-40^{\circ} \mathrm{C}$ solution of benzyl ether 35 ( $27 \mathrm{mg}, 75 \mu \mathrm{mol}, 1.0$ equiv.) in $\mathrm{CH}_{2} \mathrm{Cl}_{2}(32 \mathrm{~mL}, 0.025 \mathrm{M}), \mathrm{BCl}_{3}$ (1.0 M in $\mathrm{CH}_{2} \mathrm{Cl}_{2}, 0.37 \mathrm{~mL}, 0.37 \mathrm{mmol}, 5.0$ equiv.) was added dropwise. The stirred reaction mixture was slowly warmed to $-20^{\circ} \mathrm{C}$ over 2 hours and stirred for 1 more hour at $-20^{\circ} \mathrm{C}$, at which point methanol $(0.10 \mathrm{~mL}) \mathrm{was}$ added. The cooling bath was removed and stirring continued for 30 minutes. The volatiles were removed under reduced pressure. Purification by flash chromatography (Biotage, C18 reverse phase, Water/MeOH) provided the nucleoside 37 as a white foam (16 mg, 79\% yield): $[\alpha]_{\mathrm{D}}^{25}+20(c=0.4, \mathrm{MeOH})$; Molecular formula: $\mathrm{C}_{11} \mathrm{H}_{16} \mathrm{~N}_{2} \mathrm{O}_{6}$; MW: 272.26; IR (neat) $v_{\max } 3369,1681 \mathrm{~cm}^{-1}$; ${ }^{1} \mathrm{H} \mathrm{NMR}\left(500 \mathrm{MHz}, \mathrm{CD}_{3} \mathrm{OD}\right) \delta 8.12(\mathrm{~d}, J 8.1 \mathrm{~Hz}, 1 \mathrm{H})$, $5.90(\mathrm{~d}, J 6.6 \mathrm{~Hz}, 1 \mathrm{H}), 5.71(\mathrm{~d}, J 8.0 \mathrm{~Hz}, 1 \mathrm{H}), 4.21(\mathrm{~d}, J 6.6 \mathrm{~Hz}, 1 \mathrm{H}), 3.88-3.85(\mathrm{~m}, 1 \mathrm{H}), 3.85-3.76(\mathrm{~m}, 2 \mathrm{H}), 3.68$ $(\mathrm{d}, J 11.1 \mathrm{~Hz}, 1 \mathrm{H}), 3.55(\mathrm{~d}, J 11.1 \mathrm{~Hz}, 1 \mathrm{H}), 1.16(\mathrm{~s}, 3 \mathrm{H}) \mathrm{ppm}, \mathrm{OH}$ and $\mathrm{NH}$ signals missing possibly due to exchange in $\mathrm{CD}_{3} \mathrm{OD} ;{ }^{13} \mathrm{C}$ NMR $\left(126 \mathrm{MHz}, \mathrm{CD}_{3} \mathrm{OD}\right) \delta 166.7,153.3,142.9,102.9,90.1,88.7,77.4,65.4,62.7,48.7,17.7$ ppm; HRMS calcd for $\mathrm{C}_{11} \mathrm{H}_{16} \mathrm{~N}_{2} \mathrm{O}_{6} \mathrm{Na}\left[\mathrm{M}+\mathrm{Na}^{+}\right]$: 295.0901; found 295.0902 (+0.3 ppm).

\section{Acknowledgements}

Funding for this research has been granted from Natural Sciences and Engineering Research Council (NSERC) and Canadian Glycomics Network (Glyconet http://10.13039/501100009056). This research was enabled in part by WestGrid (www.westgrid. ca) and Compute Canada-Calcul Canada (www. computecanada.ca).

\section{Supplementary Material}

The supporting information is available free of charge on the website and contains proof of structures, X-ray crystallographic data for compound S20a, ${ }^{1} \mathrm{H}$ NMR and ${ }^{13} \mathrm{C}$ NMR spectra for all new compounds along with details concerning the computational method, energies, and Cartesian coordinates for intermediate $\mathbf{E}$. 


\section{References}

1. Jordheim, L. P.; Durantel, D.; Zoulim, F.; Dumontet, C. Nat. Rev. Drug Discovery 2013, 12, 447-464. http://dx.doi.org/10.1038/nrd4010.

2. Seley-Radtke, K. L.; Yates, M. K. Antiviral Res. 2018, 154, 66-86. http://dx.doi.org/10.1016/j.antiviral.2018.04.004.

3. Tsesmetzis, N.; Paulin, C. B. J.; Rudd, S. G.; Herold, N. Cancers 2018, 10, 240. http://dx.doi.org/10.3390/cancers10070240.

4. Asselah, T. Expert Opin. Pharmacother. 2014, 15, 121-130. http://dx.doi.org/10.1517/14656566.2014.857656.

5. Guindon, Y.; Mochirian, P.; Nemer, M.; Prévost, M. Nucleoside Analogues and Methods of use thereof, 2017, PCT/CA2017/051096.

6. Guindon, IRCM, Patent US8361988B2 (2013) and US8846636B2 (2014) US 62/218,220 (Sept 2015)

7. Tambutet, G.; Becerril-Jimenez, F.; Dostie, S.; Simard, R.; Prevost, M.; Mochirian, P.; Guindon, Y. Org. Lett. 2014, 16, 5698-5701. http://dx.doi.org/10.1021/ol502777r.

8. Dostie, S.; Prevost, M.; Mochirian, P.; Tanveer, K.; Andrella, N.; Rostami, A.; Tambutet, G.; Guindon, Y. J. Org. Chem. 2016, 81, 10769-10790. http://dx.doi.org/10.1021/acs.joc.6b01845.

9. Panda, A.; Satpati, S.; Dixit, A.; Pal, S. RSC Adv., 2016, 6, 11233-11239. http://dx.doi.org/10.1039/c5ra26416b.

10. Panda, A.; Islam, S.; Santra, M.; Pal, S. RSC Adv., 2015, 5, 82450-82459. http://dx.doi.org/10.1039/c5ra19080k.

11. Sells, T.; Nair, V. Tet. Lett., 1993, 34, 3527-3530. https://dx.doi.org/10.1016/S0040-4039(00)73627-6.

12. Zong, G.; Yan, X.; Bi, J.; Jiang, R.; Qin, Y.; Yuan, H.; Lu, H.; Dong, Y.; Jin, S.; Zhang, J. PLoS One 2017, 12, e0181646.

http://dx.doi.org/10.1371/journal.pone.0181646.

13. Sun, J.; Dou, Y.; Ding, H.; Yang, R.; Sun, Q.; Xiao, Q. Mar Drugs 2012, 10, 881-889. http://dx.doi.org/10.3390/md10040881.

14. Reddy, E. R.; Trivedi, R.; Sudheer Kumar, B.; Sirisha, K.; Sarma, A. V.; Sridhar, B.; Prakasham, R. S. Bioorg. Med. Chem. Lett. 2016, 26, 3447-3452. http://dx.doi.org/10.1016/j.bmcl.2016.06.049.

15. Gonçalves-Pereira, R.; Pereira, M.; Serra, S.; Loesche, A.; Csuk, R.; Silvestre, S.; Costa, P.; Conceição Oliveira, M.; Xavier, N. Eur. J. Org. Chem. 2018, 2667-2681. http://dx.doi.org/10.1002/ejoc.201800245.

16. Guindon, Y.; Houde, K.; Prevost, M.; Cardinal-David, B.; Landry, S. R.; Daoust, B.; Bencheqroun, M.; Guerin, B. J. Am. Chem. Soc. 2001, 123, 8496-8501. https://dx.doi.org/10.1021/ja010805m.

17. Brazeau, J. F.; Mochirian, P.; Prevost, M.; Guindon, Y. J. Org. Chem. 2009, 74, 64-74. http://dx.doi.org/10.1021/j08021583.

18. Kita, Y.; Yasuda, H.; Tamura, O.; Itoh, F.; Ya, Y. K.; Tamura, Y. Tetrahedron Lett. 1985, 26, 5777-5780. https://dx.doi.org/10.1016/S0040-4039(00)98924-X.

19. Dong, S.; Parker, G. D.; Tei, T.; Paquette, L. A. Org. Lett. 2006, 8, 2429-2431. 
http://dx.doi.org/10.1021/ol060827j.

20. Paquette, L. A.; Parker, G. D.; Tei, T.; Dong, S. J. Org. Chem. 2007, 72, 7125-7134.

http://dx.doi.org/10.1021/jo070861r.

21. Paquette, L. A.; Parker, G. D.; Tei, T.; Dong, S. J. Org. Chem. 2009, 74, 1812.

http://dx.doi.org/10.1021/jo9001382.

22. Duplessis, M.; Waltz, M. E.; Bencheqroun, M.; Cardinal-David, B.; Guindon, Y. Org. Lett. 2009, 11, 31483151.

http://dx.doi.org/10.1021/Ol901126y.

23. Giese, B.; Damm, W.; Wetterich, F.; Zeltz, H.G.; Rancourt, J.; Guindon, Y. Tetrahedron Lett. 1993, 34, 58855888.

https://dx.doi.org/10.1016/S0040-4039(00)73805-6.

24. Ward, D. E.; Hrapchak, M. J.; Sales, M. Org. Lett. 2000, 2, 57-60.

http://dx.doi.org/10.1021/ol991198z.

25. North, M.; Usanov, D. L.; Young, C. Chem. Rev. 2008, 108, 5146-5226.

http://dx.doi.org/10.1021/cr800255k.

26. Denmark, S. E.; Chung, W. J. J. Org. Chem. 2006, 71, 4002-4005.

http://dx.doi.org/10.1021/jo060153q.

27. Shenoy, S. R.; Smith, D. M.; Woerpel, K. A. J. Am. Chem. Soc. 2006, 128, 8671-8677.

http://dx.doi.org/10.1021/ja061110u.

28. Kurono, N.; Yamaguchi, M.; Suzuki, K.; Ohkuma, T. J. Org. Chem. 2005, 70, 6530-6532.

http://dx.doi.org/10.1021/jo050791t.

29. Yajima, T.; Okada, K.; Nagano, H. Tetrahedron 2004, 60, 5683-5693.

http://dx.doi.org/10.1016/j.tet.2004.05.020.

30. Nagano, H.; Ohkouchi, H.; Yajima, T. Tetrahedron 2003, 59, 3649-3663.

http://dx.doi.org/10.1016/S0040-4020(03)00421-6.

31. Nagano, H.; Toi, S.; Matsuda, M.; Hirasawa, T.; Hirasawa, S.; Yajima, T. J. Chem. Soc. Perkin Trans. I 2002, 2525-2538.

http://dx.doi.org/10.1039/b205613p.

32. However, $\mathrm{PMBCl}$ or $\mathrm{PMBOH}$ could not be observed in the NMR spectra.

33. Gau, H.-M.; Lee, C.-S.; Lin, C.-C.; Jiang, M.-K.; Ho, Y.-C.; Kuo, C.N. J. Am. Chem. Soc. 1996, 118, $2936-2941$. http://dx.doi.org/10.1021/ja952730q.

34. Gaussian 09, Revision D.01, M. J. Frisch, G. W. Trucks, H. B. Schlegel, G. E. Scuseria, M. A. Robb, J. R. Cheeseman, G. Scalmani, V. Barone, B. Mennucci, G. A. Petersson, H. Nakatsuji, M. Caricato, X. Li, H. P. Hratchian, A. F. Izmaylov, J. Bloino, G. Zheng, J. L. Sonnenberg, M. Hada, M. Ehara, K. Toyota, R. Fukuda, J. Hasegawa, M. Ishida, T. Nakajima, Y. Honda, O. Kitao, H. Nakai, T. Vreven, J. A. Montgomery, Jr., J. E. Peralta, F. Ogliaro, M. Bearpark, J. J. Heyd, E. Brothers, K. N. Kudin, V. N. Staroverov, T. Keith, R. Kobayashi, J. Normand, K. Raghavachari, A. Rendell, J. C. Burant, S. S. Iyengar, J. Tomasi, M. Cossi, N. Rega, J. M. Millam, M. Klene, J. E. Knox, J. B. Cross, V. Bakken, C. Adamo, J. Jaramillo, R. Gomperts, R. E. Stratmann, O. Yazyev, A. J. Austin, R. Cammi, C. Pomelli, J. W. Ochterski, R. L. Martin, K. Morokuma, V. G. Zakrzewski, G. A. Voth, P. Salvador, J. J. Dannenberg, S. Dapprich, A. D. Daniels, O. Farkas, J. B. Foresman, J. V. Ortiz, J. Cioslowski, and D. J. Fox, Gaussian, Inc., Wallingford CT, 2013.

35. Zhao, Y.; Truhlar, D. G. Acc. Chem. Res. 2008, 41, 157-167.

http://dx.doi.org/10.1021/ar700111a.

36. Cossi, M.; Scalmani, G.; Rega, N.; Barone, V. J. Chem. Phys. 2002, 117, 43-54. 
http://dx.doi.org/10.1063/1.1480445.

37. Godin, F.; Duplessis, M.; Buonomano, C.; Trinh, T.; Houde, K.; Chapdelaine, D.; Rodrigue, J.; Boutrosa, A.; Guindon, Y. Org. Chem. Front., 2014, 1, 974-982.

http://dx.doi.org/10.1039/c4qo00142g.

38. Kang, M.; Park, J.; Pedersen, S. F. Synlett 1997, 41-43.

http://dx.doi.org/10.1055/s-1997-709.

39. Park, J.; Pedersen, S. F. J. Org. Chem. 1990, 55, 5924-5926.

http://dx.doi.org/10.1021/jo00311a002.

40. Chapdelaine, D.; Cardinal-David, B.; Prevost, M.; Gagnon, M.; Thumin, I.; Guindon, Y. J. Am. Chem. Soc. 2009, 131, 17242-17245.

http://dx.doi.org/10.1021/ja905452f.

41. Prevost, M.; Dostie, S.; Waltz, M. E.; Guindon, Y. J. Org. Chem. 2014, 79, 10504-10525.

http://dx.doi.org/10.1021/j0502181a.

42. Solsona, J.; Romea, P.; Urpi, F.; Vilarrasa, J. Org. Lett. 2002, 4, 519-522.

http://dx.doi.org/10.1021/ol0274054.

43. De Esch, I. J.; Gaffar, A.; Menge, W. M.; Timmerman, H. Bioorg. Med. Chem. 1999, 7, 3003-3009. https://dx.doi.org/10.1016/S0968-0896(99)00253-9.

44. Maulucci, N.; Izzo, I.; Bifulco, G.; Aliberti, A.; De Cola, C.; Comegna, D.; Gaeta, C.; Napolitano, A.; Pizza, C.; Tedesco, C.; Flot, D.; De Riccardis, F. Chem. Commun. (Camb) 2008, 3927-3929. http://dx.doi.org/10.1039/b806508j.

45. Iseki, K.; Kuroki, Y.; Asada, D.; Takahashi, M.; Kishimoto, S.; Kobayashi, Y. Tetrahedron 1997, 53, $10271-$ 10280.

https://dx.doi.org/10.1016/S0040-4020(97)00683-2. 